\author{
Universidade de São Paulo \\ Instituto de Astronomia, Geofísica e Ciências Atmosféricas
}

Departamento de Astronomia

\author{
Johnny Hebert Esteves de Queiroz
}

\title{
A Correlação entre AGNs e Aglomerados de Galáxias Através do Óptico e Raios-X
}



Johnny Hebert Esteves de Queiroz

\section{A Correlação entre AGNs e Aglomerados de Galáxias Através do Óptico e Raios-X}

Dissertação apresentada ao Departamento de Astronomia do Instituto de Astronomia, Geofísica

e Ciências Atmosféricas da Universidade de São Paulo como requisito parcial para a obtenção do título de Mestre em Ciências.

Área de Concentração: Astronomia

Orientador(a): Prof. Gastão B. Lima Neto

Versão Corrigida. O original encontrase disponível na Unidade.

São Paulo 2019 

À minha mãe... 



\section{Agradecimentos}

Ao meu orientador, Prof. Dr. Gastão Lima Neto, pela sua paciência e dedicação à minha formação.

À Profa. Dr. Marcelle Soares-Santos pela oportunidade riquíssima de colaboração internacional. À todos pesquisadores em "Brandeis University" - EUA, que me acolheram com estima. Ào pesquisador Felipe Andrade-Santos que me apresentou a comunidade cientifica de raios-X em "Harvard-Smithsonian CFA".

À minha mãe, Maria, ao meu pai, João e à minha irmã, Thainy. Sem elas nada disso teria sido possível. Em especial, a minha mãe, que foi gigante perante as dificuldades do mundo.

Agradeço com muito amor e alegria a todos meus amigos e amigas do IAG, por terem me presenteado com tamanho companheirismo.

Aos Professores do Departamento de Astronomia do IAG, que contribuíram de maneira enriquecedora para minha formação.

Aos pesquisadores Tatiana Bastos e Antônio de Carvalho que me apoiaram nessa jornada, e na revisão dessa tese.

Aos amigos da moradia estudantil, CRUSP, que fizeram da Universidade minha casa e minha família.

A todos amigos da USP que compuseram o significado de Universidade na sua forma mais ampla.

Aos programas de permanência estudantil da Universidade de São Paulo, essenciais para a minha dedicação aos estudos.

À FAPESP, pelo apoio financeiro no projeto de mestrado, $n^{o}$ : 2016/25599-0, e no projeto BEPE, no: 2018/09592-1. À CAPES pelo apoio financeiro ao programa. 
Esta tese/dissertação foi escrita em $\mathrm{IAT}_{\mathrm{E}} \mathrm{X}$ com a classe IAGTESE, para teses e dissertações do IAG. 
"Da minha aldeia vejo quanto da terra se pode ver do Universo... Por isso a minha aldeia é tão grande como outra terra qualquer, Porque eu sou do tamanho do que vejo E não do tamanho da minha altura..." "O Guardador de Rebanhos"em Poemas de Alberto Caeiro Fernando Pessoa. 



\section{Resumo}

O principal objetivo deste trabalho é investigar a relação dos efeitos ambientais de aglomerados de galáxias com a atividade de galáxias de núcleo ativo (AGN). Além disso, revisitamos relações ambientais entre o aglomerado e a sua população galáctica como um todo. Utilizando um catálogo de 96 aglomerados com dados em raios-X do satélite Chandra e com observações no óptico do DES e SDSS, selecionamos cerca de 1000 AGNs em raios-X.

Observamos a conhecida dependência das AGNs com o ambiente quente de aglomerados. A fração de AGNs, $f_{\text {AGNs }}$, é menor no centro dos aglomerados e tem um pico um pouco depois de $R_{200, c}$. Além disso, a $f_{\mathrm{AGNs}}$ em hospedeiras azuis e vermelhas ao longo do raio do aglomerado apresenta um comportamento similar a segregação morfológica da população galáctica.

Do ponto de vista evolutivo, encontramos um efeito Butcher-Oemler das AGNs. Observamos um crescimento da $f_{\text {AGNs }}$ azuis de $\sim 0.2 \mathrm{em} z=0.1$ até $\sim 0.5 \mathrm{em} z=0.65$. Neste sentido, apontamos para um cenário onde a pressão de arraste e o estrangulamento são os mecanismos responsáveis pela evolução da fração de AGNs observada.

Em relação aos aglomerados cool-core $(\mathrm{CC})$, observamos uma anti-correlação da $f_{\mathrm{AGNs}}$ com a intensidade do CC. Apontamos três cenários possíveis para essa observação: (i) a pressão de arraste é mais eficiente em sistemas CC, as galáxias tipo disco perdem seu gás antes de chegar ao centro do aglomerado, devido ao esgotamento do reservatório de gás, temos uma fração menor de AGNs; (ii) aglomerados CC são mais dinamicamente relaxados, não possuem um histórico recente de acreção, as galáxias já perderam todo seu gás frio; (iii) o cooling-flow é contrabalanceado não apenas pela AGN central mas, também, por toda população de AGNs do aglomerado. Esses cenários podem ser testados em simulações hidrodinâmicas cosmológicas. 



\section{Abstract}

The main aim of this work is to probe the environmental relationships between AGN activity and galaxy cluster's (GC) environment. In addition, we look at the environmental effects on the galaxy member population as a whole.

We use a catalog of 96 GC with both X-ray data (Chandra satellite) and optical observations (SDSS and DES). We select about 1000 AGNs in X-ray.

We observed the well-known AGN dependence on the hot cluster environment. In particular, the AGN fraction $\left(f_{\mathrm{AGNS}}\right)$ is smaller in the center of the clusters and has a peak beyond the $R_{200, c}$. The fraction of the blue and red AGN hosts along the cluster radius shows a similar trend to the morphological segregation.

From an evolutionary point of view, we find an AGN Butcher-Oemler effect. We observed a growth of the $f_{\mathrm{AGNs}, \mathrm{blue}}$ from $\sim 0.2$ in $z=0.1$ up to $\sim 0.5$ in $z=0.65$. Therefore, we suggest a scenario where ram-pressure stripping and strangulation are the main mechanisms responsible for the observed AGN fraction evolution.

Regarding the cool-core (CC) systems, we observed an anti-correlation between the $f_{\mathrm{AGNs}}$ and the CC strength. We suggest three scenarios: (i) ram-pressure stripping is efficient in CC systems, the disk galaxies lose their gas before reaching the center of the cluster, this effect depletes their gas reservoir, so there is a smaller occurrence of AGNs; (ii) CC clusters are more dynamic relaxed systems, they do not show a recent accretion episode, the galaxy therein lost almost all the cool gas, hence have a smaller AGN fraction; (iii) the cooling-flow is counterbalanced not only by the central AGN but also by the entire population of AGNs in the cluster. These scenarios might be tested on cosmological hydrodynamical simulations. 



\section{Lista de Figuras}

1.1 Simulação cosmológica do projeto Millennium. Vemos o campo de densidade projetada para uma fatia de $15 \mathrm{Mpc} / h$ de espessura da saída $z=0$. Os painéis sobrepostos aumentam o zoom por fatores de 4 em cada caso, ampliando as regiões indicadas pelos quadrados brancos. Fonte: Millennium home page: https://wwwmpa.mpa-garching.mpg.de/galform/virgo/millennium/. 29

1.2 Aglomerado Abell 370. Fonte: NASA APOD 2017. . . . . . . . . . . . 31

1.3 Classificação Morfológica de Hubble. As elípticas são subdividas de E0 a E7, em uma relação crescente de elipticidade. As galáxias S0, as lenticulares, apresentam características de ambas as classificações. As espirais S são subdividas em ordem decrescente do tamanho do bojo em relação ao seu tamanho. Além disso, são dividas em duas sequências, sem barras (parte superior) e barradas (parte inferior). Por fim, as irregulares Irr, não possuem bojo nem forma característica. . . . . . . . . . . . . . 34

1.4 Histórico de formação estelar para galáxias elípticas e espirais. Fonte: Bastos $(2017$ 
1.5 A galáxia ESO 137-001 do tipo tardio caindo no aglomerado de galáxias Abell 3627. O meio intraglomerado (ICM) atua como um vento no meio interestelar (ISM) da galáxia, e o arranca para fora através da remoção por pressão de arraste. A cauda de $\mathrm{H}-\alpha$ e raios-X deixada para trás tem por volta de $80 \mathrm{kpc}$ de comprimento. A imagem é composta no óptico e em raiosX, por observações do HST e Chandra, respectivamente. Créditos: RaiosX: NASA/CXC/UAH/M.Sun et al; Optico: NASA, ESA, \& the Hubble Heritage Team $(\mathrm{STScI} / \mathrm{AURA}) \ldots \ldots \ldots \ldots$

1.6 Processos de pressão de arraste (a) e estrangulamento em galáxias (b). Em (a) a remoção do gás acontece de forma rápida, em $t=t_{q}$, resultando em uma evolução passiva. Em (b) a galáxia perde seu reservatório de gás ao ser adicionada em um ambiente de alta densidade em $t=t_{q}$. A formação estelar não é interrompida e se mantêm até que todo o conteúdo de gás presente na galáxia se esgote. Fonte Peng et al. (2015) . . . . . . . . . . .

1.7 Relação entre o tipo morfológico e a densidade local para galáxias em aglomerados. O histograma no alto de cada painel mostra o número de galáxias para cada intervalo de densidade superficial. Para os aglomerados do HST $(0.36<z<0.57)$, o histograma hachurado do alto mostra a distribuição de galáxias após correção da contaminação de galáxias na linha de visada. Os dois gráficos da esquerda são dados de Dressler (1980); Dressler et al. (1997) e da direita são de Smith et al. $(2005)$ com dados adicionais até $z \approx 1$.

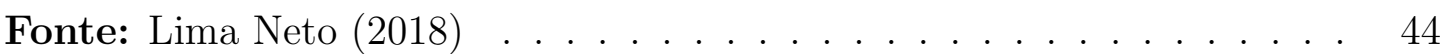

2.1 Satélite Chandra - Visão panorâmica dos instrumentos. Fonte: http:// chandra.harvard.edu/about/spacecraft.html ......... 47

2.2 Esquema do arranjo de detectores ACIS. O arranjo dos 4 chips de ACIS-I tem um campo de visão por volta de $16 \times 16 \operatorname{arcmin}^{2}$ e $1024^{2}$ pixeis. Os chips S1 e S3 usam o sistema chamado back-illuminated, o que os torna mais sensíveis; os outros chips são front-illuminated. Fonte: Andrade-Santos (2008) 48

2.3 Interface do CSCview . . . . . . . . . . . . . . . . 50 
2.4 Imagens simuladas em $1.49 \mathrm{keV}$ de detecções do Chandra-HRMA. As fontes são mostradas como apareceriam no céu, em três ângulos em relação ao eixo óptico $\left(5^{\prime}, 10^{\prime}\right.$ e $\left.15^{\prime}\right)$ e vários azimutes. Fonte:http://cxc.harvard.edu/ proposer/POG/html/chap4.html ................ 51

2.5 Distribuições de redshift, massa e temperatura. Nossa amostra conta com um total de 96 aglomerados, sendo composta pelos dados fotométricos dos SDSS e DES para $z<0.35$ (linha tracejada) e apenas dados do DES acima desse intervalo de redshift. . . . . . . . . . . . . . . . . .

3.1 O diagrama ilustra o fluxo do algorítimo Xpipe que é dividido em quatro módulos. A entrada do algorítimo é uma tabela com informações do número de identificação do Chandra, OBSID, o redshift e nome do objeto. A saída é um catálogo com as informações de entrada mais as seguintes propriedades físicas estimadas: posição do centro, erro da posição do centro, temperatura, massa, massa do gás e indicadores do estado dinâmico, i.é. $\langle w\rangle$ e $c_{S B}$. . .

3.2 Limpeza por contaminações de flares. O gráfico da esquerda se refere ao aglomerado RX J1525+0958, OBSID=1664 e da direita ao aglomerado CLJ1334+5031, OBSID=5772. Parte superior: curva de luz em contagens por segundo em função do tempo de exposição para duas observações com o detector ACIS-I. Parte inferior: distribuição de taxa de contagens. Os pontos verdes remetem aos períodos que estão dentro de $3 \sigma$ em torno da média, enquanto, as regiões vermelhas indicam os períodos de observações descartados.

3.3 Múltiplas observações em raios-X do Aglomerado da Bala (mais conhecido como Bullet Cluster). Esquerda: imagem suavizada por um filtro Gaussiano e corrigida pelo mapa de exposição, em branco são elipses em torno das fontes pontuais. Centro: sobreposição dos mapas de exposições. Direita: sobreposições das imagens de blank-sky - usada para a subtração da contaminação de emissão de fundo. 
3.4 Determinação do centro para o aglomerado do redMaPPer de MEM_MATCH_ID= $1997 \mathrm{em} z=0.3$. O circulo de linhas tracejadas verde tem 1 minuto de arco de abertura e centro na posição do centroide de luminosidade de emissão em raios-X. Esquerda: a imagem suavizada por um filtro Gaussiano corrigida por mapa de exposição. Centro: a imagem da direita com adição de um ruído Poissoniano, posteriormente suavizada num filtro Gaussiano. Direita: é a imagem residual. Nota-se que as estruturas mais fracas mudam a forma com a adição do ruído, mas o pico é pouco afetado. . . . . . . . . . . . .

3.5 Esquerda: área efetiva dos detectores ACIS-I e ACIS-S3 (chip back illuminated mais próximo do eixo do telescópio e com melhor foco) em função da energia para uma fonte no eixo do telescópio. Direita: exemplo de uma matriz de redistribuição, aqui a RMF do detector ACIS-I, para uma observação de uma fonte no eixo do telescópio. A abscissa é o canal de energia e a ordenada é a energia do fóton. A relação entre os dois é uma probabilidade representada pela cor da figura. Fonte: Lima Neto (2009). . . . . . . . . .

3.6 Relação coluna de hidrogênio $\left(n_{H}\right)$-hardness-ratio (HR). Assumimos um modelo de lei de potência mais um modelo de absorção, com ajuda do xspec simulamos observações de HR para o Chandra. Variamos a inclinação do espectro, $\Gamma$, entre 1 e 4 estes valores correspondem as curvas da direita pra esquerda. Quanto maior $\Gamma$ menor HR. As curvas são para uma galáxia hospedeira em $z=0.1$ e $z=0.5$. Não notamos grandes diferenças com o

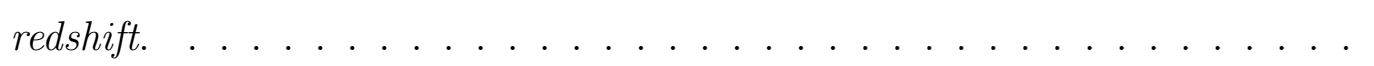

3.7 Comparação de duas estimativas de fluxo assumindo uma lei de potência mais um modelo de absorção para uma amostra seleta de fontes pontuais em raios-X. No eixo X temos o fluxo estimado pelo ajuste espectral ajustando a inclinação do espectro e a densidade de coluna de hidrogênio da galáxia hospedeira, $n_{H}$. No eixo Y temos o fluxo estimado a inclinação do espectro igual a 2 e a absorção da galáxias hospedeira não é considerada. A linha traceja em preto indica uma relação um para um e a linha vermelha é o melhor ajuste linear dos pontos. . . . . . . . . . . . . . . 
4.1 Modelo de magnitude observada ao longo do redshift para uma típica galáxia elíptica para três filtros, $r, i$ e $z \ldots \ldots \ldots \ldots$. . . . . . . . 74

4.2 Inclinação da sequência vermelha ao longo do redshift para três índices de cor. Em pontos vermelhos temos as medidas em intervalos de redshift de 0.075, e em preto as medidas dos aglomerados individuais. Usamos a amostra de 22 mil aglomerados do redMaPPer Y3 volume limitado com $\lambda>20$.

4.3 Ajuste da sequência vermelha para o aglomerado do redMaPPer de MEM_MATCH_ID= 56 em $z=0.282$. Observamos todas as galáxias dentro de $R_{500}$. O tamanho dos pontos é proporcional a $P_{m e m}$ da galáxia, escala na legenda. Os pontos em vermelho, azul e cinza são as galáxias da sequência vermelha, nuvem azul e de fundo ou fora do limite de magnitude, respectivamente. A linha tracejada vermelha é o melhor ajuste da SV. A linha tracejada azul é a magnitude limite para esse redshift. . . . . . . . . . . . . . .

4.4 Ajuste do modelo Gaussian Mixture Model (GMM) para o aglomerado do redMaPPer de MEM_MATCH_ID $=56$ em $z=0.282$, nas cores $(g-r)$ e $(r-i)$ e para galáxias dentro de $R_{500}$. Parte inferior: temos a distribuição de cor subtraída pela distribuição de galáxias de fundo, As curvas tracejadas vermelhas e azuis correspondem ao ajuste do método GMM para a sequência vermelha (SV) e a nuvem azul, respectivamente. A linha tracejada preta é $\pm 2 \sigma_{r}$ em torno da média da distribuição da SV e a linha azul claro é a cor média derivada da relação do aglomerados da amostra do redMaPPer. Parte superior: em cinza escuro temos a distribuição de galáxias ponderada por $P_{m e m}$, em vermelho claro temos as galáxias de fundo também ponderadas por $P_{\text {mem }}$ multiplicado por um termo de correção da área. A curva cinza é um ajuste gaussiano da galáxias de fundo meramente ilustrativo. . . . . . .

5.1 Perfil da fração de galaxias azuis e vermelhas em função do raio normalizado por $R_{500}$ para 14 aglomerados em redshift intermediário, $z \in(0.35,0.50)$, na amostra XMATCHA-DES. Os erros mostrados são Poissonianos. 
5.2 Comparação do perfil de fração de galaxias azuis e vermelhas com o tipo morfológico em função do raio em unidades de $M p c$. Usamos a mesma amostra da figura 5.1. As curvas com o tipo morfológico são dos 55 aglomerados e 6000 galáxias do universo local de Whitmore et al. (1993), utilizamos um sigma padrão de 0.05 a fim de comparação. O gráfico abaixo mostra a diferença da curvas em relação ao nosso trabalho. Os resultados concordam em $5 \%$ para quase todos os pontos

5.3 Evolução Fração de galaxias azuis selecionadas pela cores $(g-r)$ e $(r-i)$. Os pontos em cinza são as medidas para cada aglomerado, enquanto os quadrados azuis são as medidas em intervalos de redshift - com espessura de 0.075 .

5.4 Fração de galaxias azuis, $f_{b}$, em função das propriedades físicas do aglomerado, $M_{500}$ e $k T$, para a amostra em redshift intermediário, $0.35<z<0.65$. Os pontos em cinza são as medidas para cada aglomerado, enquanto os quadrados azuis são as medidas em intervalos de massa e temperatura. . .

5.5 Fração de galaxias azuis, $f_{b}$, em função $Y_{X}$, para a amostra em redshift intermediário, $0.35<z<0.65$. Os pontos em cinza são as medidas para cada aglomerado, enquanto os quadrados azuis são as medidas em intervalos. 84

5.6 Comparação da fração de galaxias azuis ao longo do redshift para diferentes seleções de cor. . . . . . . . . . . . . . . . . . . . 86

5.7 Comparação da relação morfologia(cor)-raio para diferentes seleções de cor. 87

5.8 Comparação da fração de galaxias azuis selecionadas pela cor $(g-r)$ para duas espessuras da sequência vermelha, fixa em 0.20 mag em preto, e ajustada em azul claro (veja seção 4.1). Parte superior: os pontos se referem aos valores de $f_{b}$ calculados para intervalos de redshift de 0.075. Parte inferior: temos a diferença entre $f_{b}$, em azul claro para cada aglomerado, em preto a diferença em intervalos de redshift. . . . . . . . . . . . . . 
6.1 Fração de AGNs ao longo do raio projetado do aglomerado em unidades de $R_{500}$. A amostra contém aglomerados um intervalo de redshift de 0.1 a 0.65 e possui 680 AGNs dividas em anéis $0.5 R_{500}$ de espessura e galáxias hospedeiras com magnitudes $M_{r} \leq-19.8$. A fração de AGNs é simplesmente o número desses objetos divido pela riqueza do aglomerado num dado anel, a riqueza é calculado assumindo o mesmo corte de luminosidade. Os erros mostrados são Poissonianos. . . . . . . . . . . . . . . . . . . . .

6.2 Fração de AGNs ao longo do raio projetado do aglomerado em unidades de $R_{500}$. A amostra é divido em dois intervalos de redshift, $0.1<z<0.45 \mathrm{e}$ $0.45<z<0.65$. Os erros mostrados são Poissonianos. . . . . . . . . . . .

6.3 Distribuição de AGNs normalizada em um ao longo do raio projetado do aglomerado em unidades de $R_{500}$. A amostra contém aglomerados um intervalo de redshift de 0.1 a 0.65 e possui 680 AGNs dividas em anéis $0.5 R_{500}$ de espessura. Os erros mostrados são Poissonianos. . . . . . . . . . . .

6.4 Perfil da fração de AGNs azuis e vermelhas em função do raio normalizado por $R_{500}$ para 468 objetos no intervalo $0.1<z<0.65$. As galáxias foram selecionadas nas cores $(g-r)$ e $(r-i)$ pelo método de contagens, com os mesmos cortes de magnitude da figura 6.5 . . . . . . . . . . . . . .

6.5 Evolução da fração de AGNs azuis dentro de $R_{200, c}$. São 262 AGNs com magnitudes $m<m^{*}+3$. As galáxias azuis foram selecionadas nas cores $(g-r)$ e $(r-i)$. Os erros mostrados são Poissonianos.

6.6 Fração de AGNs ao longo do redshift para toda galáxias hospedeiras dentro $4 R_{500}$ e com magnitudes $M_{r} \leq-19.8$. Os erros mostrados são Poissonianos.

6.7 Fração de AGNs ao longo do redshift dividas em 3 regiões do aglomerado, $R_{500}, R_{200, c}$ e $(1-4) R_{500}$. Temos as fontes pontuais em raios-X: soft e hard. As fontes hard(soft) são indicadores de AGNs (não)obscurecidas. Notamos que a evolução está associada com as AGNs mais obscurecidas e nas regiões periféricas do aglomerado. . . . . . . . . . . . . . .

6.8 Dependência da atividade nuclear com a massa do aglomerado. Fração de AGNs dentro de $R_{500}$ em função de $M_{500} \ldots \ldots$. . . . . . . . . . . . . 101

6.9 Fração de AGNs dentro de $R_{500}$ em função da concentração, $c_{S B}$ - indicador de sistemas cool-core. 
6.10 Fração de AGNs em função do desvio do centro $\langle w\rangle$ - indicador de pertubação do estado dinâmico. . . . . . . . . . . . . . . . . . . 103

6.11 Ilustração do cenário geral proposto nesse trabalho. . . . . . . . . . . 106 


\section{Lista de Tabelas}

1.1 Redshift aproximado de formação de estruturas no modelo hierárquico . . . 28

1.2 Resumo das propriedades das galáxias da sequência de Hubble. . . . . . . . 35

2.1 Amostra em raios-X e no óptico. . . . . . . . . . . . 54

5.1 Teste Estatístico de Correlação . . . . . . . . . . . . . . 85 



\section{Sumário}

1. Introdução . . . . . . . . . . . . . . . . . . . . . . 27

1.1 Formação de Estruturas em Larga Escala . . . . . . . . . . . . . . . . . . 27

1.2 Aglomerados de Galáxias . . . . . . . . . . . . . . . . . . . . . . . 30

1.2.1 Emissão em Raios-X: Meio Intra-aglomerado . . . . . . . . . . . . 32

1.2.2 Aglomerados cool-core: Cooling-Flow . . . . . . . . . . 33

1.3 Evolução de Galáxias . . . . . . . . . . . . . . . . . . . . . . 33

1.3.1 Morfologia . . . . . . . . . . . . . . . . 34

1.3.2 Formação Estelar . . . . . . . . . . . . . . . . . . . . 35

1.3.3 Processos internos . . . . . . . . . . . . . . . 36

1.3.4 Galáxias de Núcleo Ativo: AGNs . . . . . . . . . . . . 37

1.3.5 Interações Galácticas . . . . . . . . . . . . . . . . . . . 38

1.3.5.1 Fusão Galáctica . . . . . . . . . . . . . . . . . . 38

1.3.5.2 Assédio Galáctico . . . . . . . . . . . . . . . . . 39

1.4 Efeitos dos Aglomerados na Evolução de Galáxias . . . . . . . . . . . . . . 40

1.4.1 Pressão de Arraste . . . . . . . . . . . . . . . . . . . 40

1.4.1.1 Estrangulamento . . . . . . . . . . . . . . . . 42

1.4.2 Qual a influência do ambiente na evolução de galáxias? . . . . . . . 42

1.4.3 AGNs em Aglomerados . . . . . . . . . . . . . . . . 45

2. Base de dados . . . . . . . . . . . . . . . . . . . . . . 47

2.1 Astronomia em Raios-X: Chandra . . . . . . . . . . . . . . 47

2.2 Amostras . . . . . . . . . . . . . . . . . . 48

2.3 Chandra Source Catalog $(\mathrm{CSC}) \quad \ldots \ldots \ldots$. . . . . . . . . . 49 
2.4 Grandes Levantamentos Fotométricos: SDSS e DES . . . . . . . . . . . 52

2.4.1 Dark Energy Survey - DES . . . . . . . . . . . 52

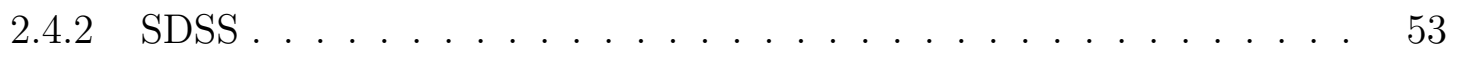

2.5 Propriedades da Amostra . . . . . . . . . . . . . . . 54

2.5.1 Catálogo de Aglomerados . . . . . . . . . . . . 554

3. Análises em raios-X: Chandra .................... . . 57

3.1 Módulo 1: redução de dados . . . . . . . . . . . . . . 58

3.2 Módulo 2: imageamento . . . . . . . . . . . . . 5 59

3.3 Módulo 3: pré-análise . . . . . . . . . . . . . . . . . . . . 61

3.3.1 Centro em Raios-X . . . . . . . . . . . . . . . 61

3.3 .2 Anéis ............................. 62

3.3.3 Arquivos Auxiliares: ARF e RMF . . . . . . . . . . . 63

3.4 Módulo 4: análise . . . . . . . . . . . . . . . . . . . . 64

3.4 .1 Ajuste Espectral . . . . . . . . . . . . . . . 64 64

3.4.2 Ajuste do Perfil de Brilho Superficial . . . . . . . . . 65 65

3.4.3 Determinação de $M_{500} \ldots \ldots \ldots \ldots$. . . . . . . . 65

3.4.4 Desvio do centro: $\langle w\rangle \ldots \ldots \ldots 6$

3.4 .5 Concentração: $c_{S B} \ldots \ldots \ldots \ldots \ldots$

3.5 Xpipe: pipeline em raios-X . . . . . . . . . . . . . . 67 67

3.6 Fontes Pontuais: AGNs . . . . . . . . . . . . . . . . . . . . . . . 68

3.6.1 AGNs Obscurecidas . . . . . . . . . . . . . . 68

3.6.2 Luminosidade em raios-X . . . . . . . . . . . . . 69

4. Análise no Óptico . . . . . . . . . . . . . . . . . . . . 771

4.1 Seleção de Galáxias Membro . . . . . . . . . . . . . . . . . . . . 71

4.1.1 Probabilidade de Redshift . . . . . . . . . . . . 77

4.1 .2 Probabilidade Radial . . . . . . . . . . . . . . 72

4.2 Distribuição de Cor . . . . . . . . . . . . . . . . . . . . . . 73

4.2 .1 Limite de magnitude . . . . . . . . . . . . 73

4.2 .2 Sequência Vermelha . . . . . . . . . . . . . . . . 73

4.2.3 Galáxias Azuis e Vermelhas . . . . . . . . . . . . . 75 
5. Evolução de Galáxias em Aglomerados . . . . . . . . . . . . . . . . . 79

5.1 Relação Cor-Raio . . . . . . . . . . . . . . . . . . . . . . . . 779

5.2 Evolução: Efeito Bucher-Oemler . . . . . . . . . . . . . . 82

5.3 Correlações: Estado Dinâmico . . . . . . . . . . . . . . . . . . 83

5.4 Efeito de Seleção: Escolha da Cor e Espessura da Sequência Vermelha . . . 86

6. AGNs em Aglomerados de Galáxias . . . . . . . . . . . . . . . . . . . . 91

6.1 A frequência de AGNs Dependem Do Meio . . . . . . . . . . . . . . 93

6.2 Efeito Butcher-Oemler das AGNs . . . . . . . . . . . . . . 96

6.3 Evolução . . . . . . . . . . . . . . . . . . . . . . . . . . . . . . . . . 9 98

6.4 O Papel Do Estado Dinâmico . . . . . . . . . . . . . . . . . . 100

6.5 Efeitos de Ambiente em AGNs . . . . . . . . . . . . . . . . . 104

7. Conclusões . . . . . . . . . . . . . . . . . . . . . . . . . 107

7.1 Perspectivas . . . . . . . . . . . . . . . . . . 110

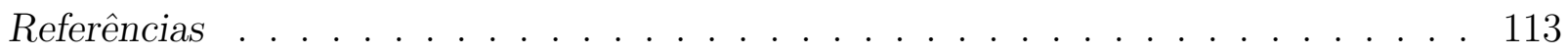

Apêndice $\quad 119$ 

Capítulo 1

\section{Introdução}

A busca por excelência da cosmologia é compreender a formação e a evolução do Universo como um todo. Muitos avanços teóricos e observacionais foram feitos desde o último século até se estabelecer o modelo $\Lambda$ CDM (CDM do inglês Cold Dark Matter e $\Lambda$ a constante cosmológica), tido como padrão atualmente.

Do ponto de vista observacional, debutamos com a descoberta de objetos celestes além da nossa galáxia, a Via Láctea, fundando assim o ramo da Astronomia Extragaláctica. Do ponto de vista teórico, com o advento da teoria da relatividade geral os primeiros modelos cosmológicos físicos se estabeleceram, regidos pela gravidade e exploraram aspectos do espaço e tempo. Em segundo momento, estudando campos de densidade não homogêneos, cosmólogos vislumbraram as sementes das estruturas em larga escala que observamos hoje.

Neste capítulo, introduzimos a formação de estruturas em larga escala e suas propriedades físicas. Fazemos uma breve revisão da evolução de galáxias. Por fim, revisamos os principais efeitos de ambiente sobre as propriedades fotométricas das galáxias.

\subsection{Formação de Estruturas em Larga Escala}

Nesta seção fazemos uma breve descrição qualitativa da formação de estruturas em larga escala no Universo.

No modelo de $\Lambda \mathrm{CDM}$, as grandes estruturas têm origem nas pertubações primordiais que estão impressas na Radiação Cósmica de Fundo em Microondas (CMB do inglês Cosmic Microwave Background). Por interagir fracamente com a radiação, a matéria escura se desacopla do plasma relativístico de matéria e radiação antes da época de recombinação. A formação de estruturas é causada pela instabilidade gravitacional, a partir de flutuações 
Tabela 1.1 - Redshift aproximado de formação de estruturas no modelo hierárquico

\begin{tabular}{|c|c|c|c|c|}
\hline $1+z$ & $M_{\text {halo }}\left[M_{\odot}\right]$ & $r_{v i r}[\mathrm{kpc}]$ & $\sqrt{\overline{v^{2}}}[\mathrm{~km} / \mathrm{s}]$ & \\
\hline$\sim 20$ & $10^{10}$ & 70 & 70 & \multirow{3}{*}{ (galáxias) } \\
\hline 16 & $10^{11}$ & 150 & 130 & \\
\hline 8 & $10^{12}$ & 300 & 200 & \\
\hline 4 & $10^{13}$ & 700 & 300 & \multirow{3}{*}{ (grupos/aglomerados) } \\
\hline 3 & $10^{14}$ & 1500 & 600 & \\
\hline 2 & $10^{15}$ & 3000 & 1000 & \\
\hline
\end{tabular}

Para um universo sem energia escura OCDM (open

CDM) $\Omega_{M}=0.3, \Omega_{\Lambda}=0$. Fonte: Lima Neto (2018)

quânticas primordiais amplificadas na época de Inflação. Na recombinação os bárions se destacam do campo de radiação e passam a se acumular nos halos de matéria escura. Por sua vez, os halos de matéria crescem cada vez mais até que se tornam tão densos que se destacam da expansão geral do Universo (fluxo de Hubble) e colapsam formando sistemas ligados. A formação de grandes estruturas ocorre da acreção desses pequenos halos de matéria escura, o cenário conhecido como Hierárquico. Em particular, assumindo um modelo de colapso esférico em Universo sem energia escura, podemos ter ideia quando esses sistemas atingem o equilíbrio gravitacional. Na tabela 1.1 temos o redshift aproximado de formação de estruturas no modelo hierárquico. Também apresentamos valores típicos da $M_{\text {halo }}$, raio do virial, $r_{v i r}$, e a velocidade quadrática média, $\sqrt{\overline{v^{2}}}$. Os resultados são para um modelo OCDM que é uma aproximação do modelo $\Lambda$ CDM para o intervalo de reshift em questão.

Nesse cenário os aglomerados são estruturas relativamente jovens- aglomerados se formam por volta de $z \sim 1$. As galáxias são estruturas mais velhas e se formam em redshifts mais altos.

Vale ressaltar que halos gravitacionalmente ligados podem estar contidos em halos maiores. Isto ocorre, por exemplo, em aglomerados de galáxias ou em galáxias individuais com galáxias satélites (como a Via Láctea). Com simulações cosmológicas podemos compreender melhor os sub-halos, por exemplo, em grandes escalas, podemos ver filamentos de matéria, e algumas regiões vazias. Na intersecção de filamentos vemos pontos mais densos que são os aglomerados ricos (como ilustrado pela Figura 1.1). Mais ainda, se reparamos podemos ver vários aglomerados ao longo de um filamento que são super-aglomerados. 


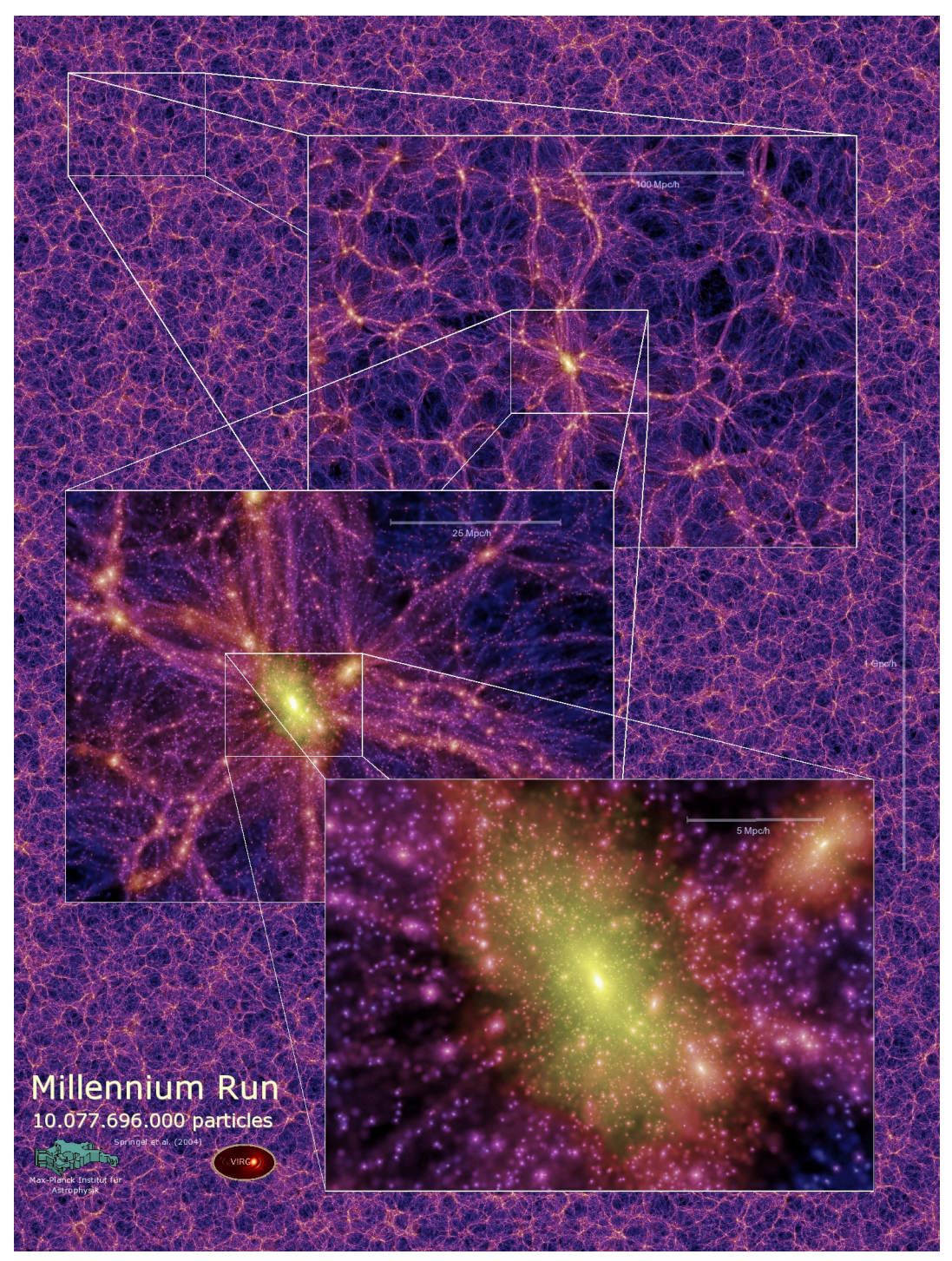

Figura 1.1: Simulação cosmológica do projeto Millennium. Vemos o campo de densidade projetada para uma fatia de $15 \mathrm{Mpc} / h$ de espessura da saída $z=0$. Os painéis sobrepostos aumentam o zoom por fatores de 4 em cada caso, ampliando as regiões indicadas pelos quadrados brancos. Fonte: Millennium home page: https://wwwmpa.mpa-garching.mpg.de/galform/virgo/millennium/.

Individualmente, aglomerados são testemunhas de uma história recente de fusões, como previsto pelo modelo de formação hierárquica de estruturas. Em um universo aberto, de baixa densidade, os aglomerados ricos não deveriam apresentar sinais de subestruturas atualmente (Lacey e Cole, 1993). Fusões com outros aglomerados e grupos são responsáveis pela formação e crescimento dos mesmos. Esses episódios perturbam o equilíbrio gravitacional do sistema, porém, o tempo em que o sistema leva até atingir novamente o equilibro (i.é. tempo de relaxação) é relativamente pequeno, é comparável a escala de tempo dinâmica que é da ordem de bilhões de anos.

Para uma introdução mais abrangente sobre o assunto e uma leitura em Português, 
recomendamos as Notas de Aula Em Astronomia Extragaláctica (Lima Neto, 2018), e para uma abordagem matemática completa veja o livro Structure Formation in the Universe (Padmanabhan, 1993).

A cosmologia assumida nesse trabalho é dada por: $\Omega_{M}=0,3, \Omega_{\lambda}=0,7$ e $H_{0}=70 \mathrm{~km}$ $\mathrm{s}^{-1} \mathrm{Mpc}^{-1}$.

\subsection{Aglomerados de Galáxias}

Na infinitude do universo, os aglomerados são as maiores estruturas que se formaram e alcançaram o equilíbrio dinâmico gravitacional. São milhares de galáxias espalhadas em uma região de dimensão da ordem de $2-3 h^{-1} \mathrm{Mpc}$ - unidas gravitacionalmente por um poço de potencial de massa efetiva tipicamente por volta de $1-10 \times 10^{14} \mathrm{M}_{\odot}$. Na Figura 1.2 temos um exemplo dessa estrutura.

Muito além de apenas um enxame de galáxias, os aglomerados de galáxias são constituídos também de gás e matéria escura. Grande parte da sua composição é matéria não bariônica (matéria escura), algo por volta de $80 \%$.

Por outro lado, a matéria bariônica surpreendentemente não está em grande parte na forma de estrelas, e sim, em um plasma difuso chamado meio intra-aglomerado, ou simplesmente ICM (da sigla em inglês intracluster medium). Sendo este observado pela sua emissão em raios-X. As galáxias contribuem bem pouco para massa do sistema, em aglomerados ricos por exemplo, apenas $\approx 2 \%$ da massa está na forma de estrelas Laganá et al., 2013).

Os aglomerados podem ser identificados no óptico, em raios-X e em Rádio. O primeiro grande catálogo desses objetos foi o de Abell contendo 2712 aglomerados ricos, selecionado opticamente utilizando placas fotográficas do Palomar Sky Survey, criado em sua primeira versão em 1958 (Abell, 1958). Recentemente com o advento dos grandes levantamentos fotométricos, a identificação é feita por algoritmos chamados de buscadores de aglomerados. Em particular, o redMaPPer na sua versão v6.4.14 encontrou 87.297 aglomerados com riqueza $\lambda>5$ (Rykoff et al., 2016). Esse buscador utiliza a relação conhecida como sequência vermelha na qual as galáxias apresentam semelhantes índices de cor. Porém, esses catálogos estão sujeitos a alguns vieses. Destaco efeitos de projeção que super estimam a riqueza do aglomerado. Além desse, a medida que o redshift aumenta, a quantidade de 


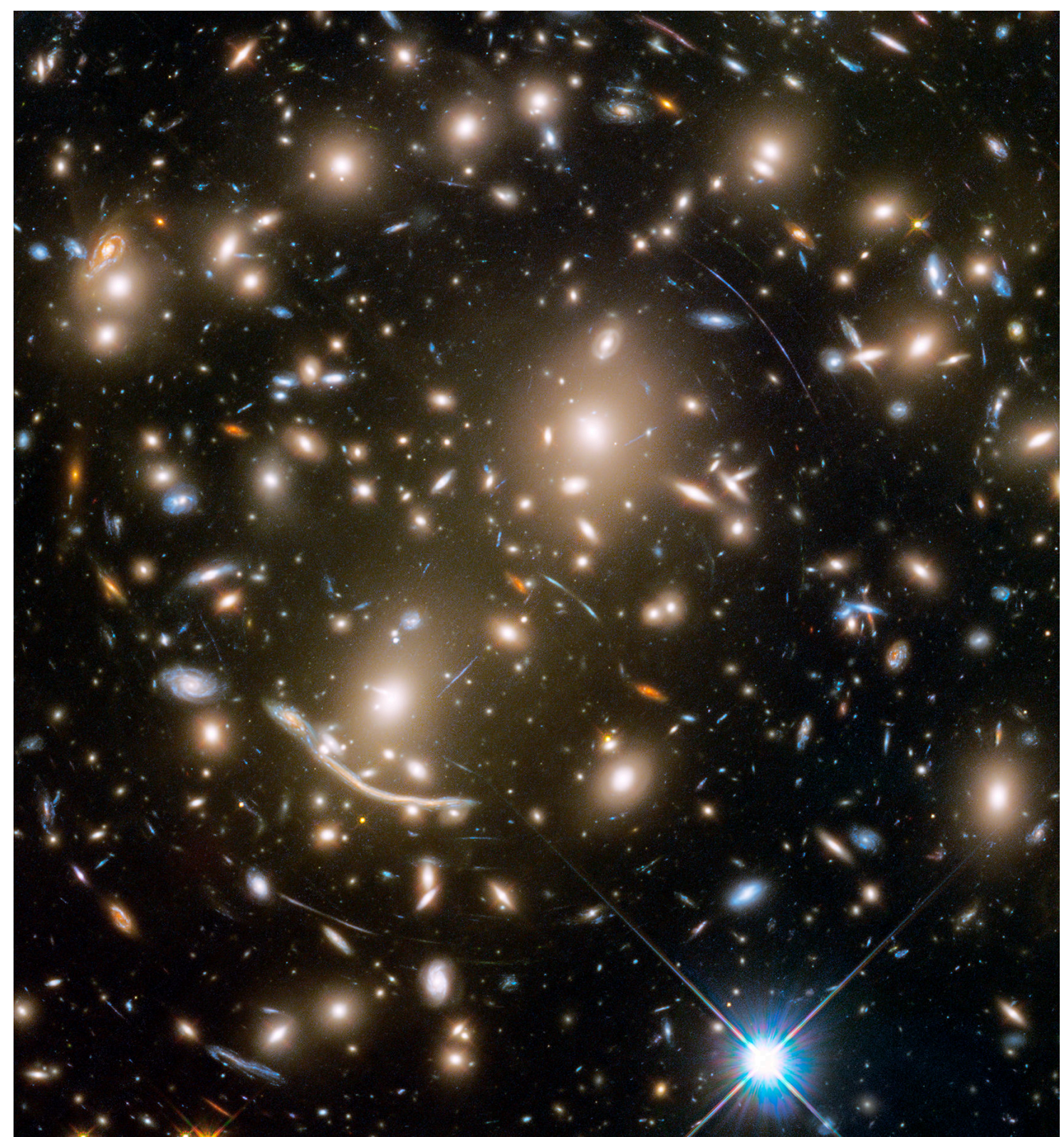

Figura 1.2: Aglomerado Abell 370. Fonte: NASA APOD 2017.

aglomerados pobres detectados diminui - consequência do viés de Malmquist.

A detecção de aglomerados em raios-X tem algumas vantagens em relação ao óptico, uma delas é de não estar sujeito a efeito de projeções. No entanto, a sua observação é muito mais cara, pois, não pode ser feita na superfície, devido à profundidade óptica elevada da atmosfera da Terra nesse comprimento de onda. Consequentemente, a primeira observação em raios-X de uma fonte extensa extragaláctica foi feita por detectores em balões (Boldt et al., 1966; Felten et al., 1966). Essa fonte foi identificada como o aglomerado de Coma e confirmada posteriormente com o lançamento do satélite Uhuru (Gursky et al., 
1971). Atualmente os dois principais observatórios em raios-X utilizados para observação de aglomerados são os satélites Chandra e XMM-Newton.

Em rádio os aglomerados são detectados pelo o efeito Sunayev-Zeldovich (SZE; veja Birkinshaw, 1999). O espalhamento Compton inverso dos fótons da radiação cósmica de fundo por elétrons térmicos do ICM gera esse efeito.

\subsubsection{Emissão em Raios-X: Meio Intra-aglomerado}

O ICM encontra-se no meio entre as galáxias, onde parece ser vazio quando olhamos uma imagem no visível. Surpreendentemente, a sua massa corresponde a cerca de $13 \%$ $16 \%$ da massa total, apesar de ser um plasmo difuso e rarefeito com temperaturas superiores a $2 \mathrm{keV}\left(T \geq 10^{7}-10^{8} \mathrm{~K}\right)$. Além do plasma quente, há também partículas relativísticas (raios cósmicos) e campos magnéticos no meio intra-aglomerado.

A origem da emissão do ICM é bremsstrahlung. Este por sua vez, pode ser tratado como um plasma ionizado em equilíbrio. Se considerarmos uma distribuição maxwelliana da velocidade dos elétrons é possível mostrar que a emissividade bremsstrahlung (Rybicki e Lightman, 1986), $\epsilon_{\nu}$, é dada por:

$$
\epsilon_{\nu} \equiv \frac{d E}{d V d t d \nu}=6.8 \times 10^{-38} Z^{2} n_{e} n_{i} T^{-1 / 2} e^{h \nu / k T} g(E, T) \operatorname{erg~s}^{-1} \mathrm{~cm}^{-3} \mathrm{~Hz}^{-1},
$$

onde $T$ é a temperatura do gás, $n_{e}$ é a densidade eletrônica e $g(E, T)$ é o fator de gaunt. O fator de gaunt leva em conta os efeitos quânticos e relativísticos do espalhamento bremsstrahlung. Em uma primeira aproximação, o fator de gaunt é por volta de 1.2 para a emissão de aglomerados de galáxias.

Integrando a emissão ao longo do espectro temos a emissão em raios-X, considerando $n_{e} n_{i} \approx n_{e}^{2}$ podemos aproximar a equação para:

$$
\epsilon=\frac{d E}{d V d t}=1.4261 \times 10^{-27} n_{e}^{2} T^{1 / 2} Z^{2} g(T) \mathrm{erg} \mathrm{s}^{-1} \mathrm{~cm}^{-3} .
$$

O número de fótons que chegam na Terra é bastante baixo (se comparado com as observações no óptico). Para um fluxo de $10^{11} \mathrm{erg} \mathrm{s}^{-1} \mathrm{~cm}^{-2}$, que corresponde a um aglomerado brilhante, isto se traduz em cerca de 1 fóton a cada 5 minutos por $\mathrm{cm}^{2}$

Diferentemente de outros tipo de emissão a intensidade da emissão em raios-X depende fortemente da densidade do plasma $\left(\epsilon \propto n_{e} n_{i} \approx n_{e}^{2}\right)$. Um fenômeno consequente dessa dependência é o cooling-flow(fluxo de resfriamento, explicado na seção seguinte). 


\subsubsection{Aglomerados cool-core: Cooling-Flow}

O cooling-flow ocorre devido ao tempo de resfriamento do gás para a região central ser menor que a idade do sistema em geral. Ou seja, o gás tem tempo suficiente para resfriar-se significativamente no centro criando assim um gradiente de temperatura. Com a região central fria e regiões externas quentes ocorre um fluxo de matéria em direção ao centro do poço potencial. Aumentando assim a densidade central do gás e por conseguinte um aumento da taxa de resfriamento (veja Fabian, 1994, para uma revisão)

O cenário clássico previa uma taxa de depósito de massa na região central de aproximadamente $530 \mathrm{M}_{\odot} /$ ano. O que suscita dois grandes problemas:

- o alto fluxo de gás frio implica numa alta taxa de formação estelar e/ou acúmulo de gás molecular frio na periferia da galáxia central;

- Linhas de emissão com energia $\leq 1 \mathrm{keV}$ são previstas nesse cenário, porém observações de alta resolução espectral não as identificaram (Peterson et al., 2001. 2003).

Os aglomerados cool-core possuem emissões em raios-X significativas no seu caroço em comparação com os não cool-core graças ao cooling-flow.

Um fato observacional importante dos aglomerados cool-core é que a temperatura central é aproximadamente 1/3 da temperatura média do aglomerado. Esta observação revela um mecanismo de feedback, que regula a temperatura central. A AGN central do aglomerado é um candidato forte para controlar o feedback.

No entanto, em casos extraordinários de aglomerados cool-core como por exemplo, M87 e Fênix, é observado também estruturas filamentares de gás frio ao redor da galáxia central Forman et al. (2007); McDonald et al. (2019).

\subsection{Evolução de Galáxias}

Nesta seção fazemos um breve introdução dos tipos de galáxias e suas propriedades. Descrevemos alguns processos importantes na evolução desses objetos celestes. 


\subsubsection{Morfologia}

A diversidade das formas em que as galáxias se apresentam no Universo é fruto da complexidade que tais sistemas possuem. Porém, numa infinidade de objetos, é muito provável encontrar características comuns que refletem propriedades físicas. Nesse sentido, a construção de um esquema de classificação é uma forma de agrupar essas semelhanças.

De fato, tomemos como exemplo o sistema de classificação morfológica de Hubble 1926 . 1936) que é representado pelo diagrama de diapasão (tunefork) - Figura 1.3. Por razões históricas, as galáxias a esquerda do diagrama são ditas "joviais" (do inglês early-type) e a direita "tardia" (do inglês late-type). As galáxias elípticas (E) possuem uma forma oval, enquanto as espirais (S) possuem uma forma de disco, com padrões espirais e as lenticulares (S0) são uma classificação entre esses dois tipos, introduzida posteriormente. As irregulares não possuem uma forma bem definida.

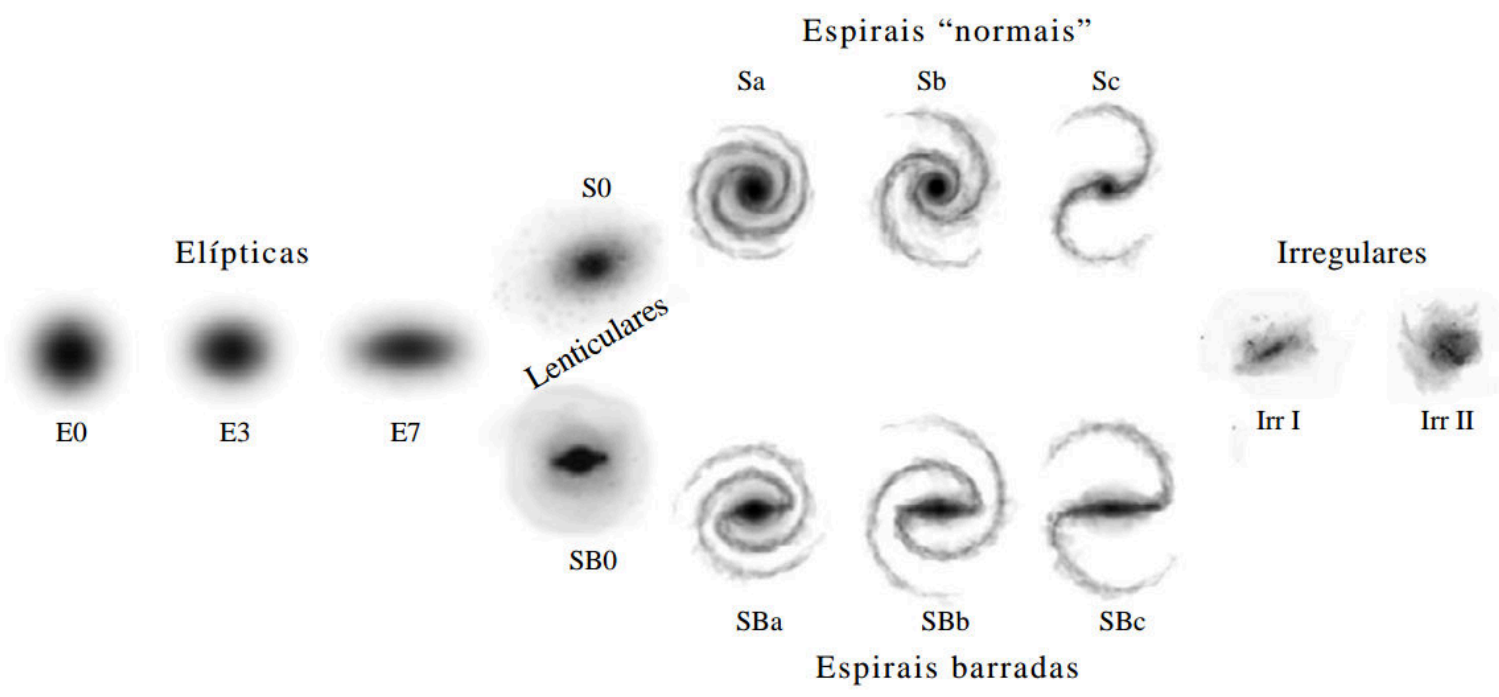

Figura 1.3: Classificação Morfológica de Hubble. As elípticas são subdividas de E0 a E7, em uma relação crescente de elipticidade. As galáxias S0, as lenticulares, apresentam características de ambas as classificações. As espirais S são subdividas em ordem decrescente do tamanho do bojo em relação ao seu tamanho. Além disso, são dividas em duas sequências, sem barras (parte superior) e barradas (parte inferior). Por fim, as irregulares Irr, não possuem bojo nem forma característica.

As espirais possuem um bojo no centro; tem uma estrutura de disco que é mantida pela rotação da galáxia; e podem possuir braços espirais bem definidos ou não. As galáxias E possuem basicamente uma estrutura esferoidal enquanto as Irr não possuem nenhuma estrutura marcante. O tamanho e a massa das galáxias varia com o tipo morfológico, galáxias tipo jovial podem ter tamanhos muito maiores que as tipo tardio. E as galáxias 
elípticas são as que tem maior massa enquanto as irregulares são as de menor massa.

Além das diferenças na forma, os tipos morfológicos também indicam propriedades físicas distintas que compõem esses sistemas. Nesse sentido, foram descobertas várias propriedades que se alteram sistematicamente nesse diagrama, veja a Tabela 1.2 para um resumo. Galáxias espirais possuem uma população de estrelas jovens no disco, geralmente são abundantes em gás frio e possuem bastante poeira criada pelas explosões de supernovas. As galáxias elípticas e a maioria das lenticulares não apresentam indícios de formação estelar recente e são compostas essencialmente por estrelas velhas.

Essas diferenças refletem na cor das galáxias, sendo esta um bom indicador morfológico. De fato, a cor amarela ou vermelha é típica de estrelas como o sol, tipo espectral G, enquanto a cor azul é tipica de estrelas tipo $\mathrm{O}$ e B. Apesar das espirais terem os dois tipos de população estelar, como as estrelas $\mathrm{O}$ e B são muito mais luminosas, estas ofuscam o brilho da população de estrelas mais velha. Outro fator importante é que as estrelas $\mathrm{O}$ e B vivem por um tempo muito menor que as tipos $G$, e geralmente morrem mais ou menos na região onde nascem, no caso das espirais, o disco.

Tabela 1.2 - Resumo das propriedades das galáxias da sequência de Hubble.

\begin{tabular}{l|lll}
\hline Propriedade & Espirais & Elípticas & Irregulares \\
\hline Massa $\left(M_{\odot}\right)$ & $10^{9}$ a $10^{12}$ & $10^{7}$ a $10^{13}$ & $10^{8}$ a $10^{11}$ \\
Diâmetro $(\mathrm{kpc})$ & $5-30$ & $1-100$ & $1-10$ \\
Luminosidade $\left(L_{\odot}\right)$ & $10^{8}$ a $10^{11}$ & $10^{6}$ a $10^{12}$ & $10^{7}$ a $10^{9}$ \\
População Estelar & Velha e Jovem & Velha & Velha e Jovem \\
Gás & Bastante & Muito pouco & Bastante \\
Poeira & Bastante & Muito pouca & Depende \\
Cor & Azulada nos braços & Amarelada/vermelha & Azulada \\
& Amarelada no bojo & & \\
\hline
\end{tabular}

Fonte: Bahcall (1999).

\subsubsection{Formação Estelar}

Como vimos na seção anterior podemos relacionar a cor de uma galáxia à população estelar presente. Vimos que em geral galáxias vermelhas são dominadas por estrelas velhas, pobres em gás frio e em poeira. Enquanto, as galáxias azuis possuem estrelas jovens, são ricas em gás frio e possuem poeira. Além disso, o bojo das espirais é composto por estrelas velhas. 


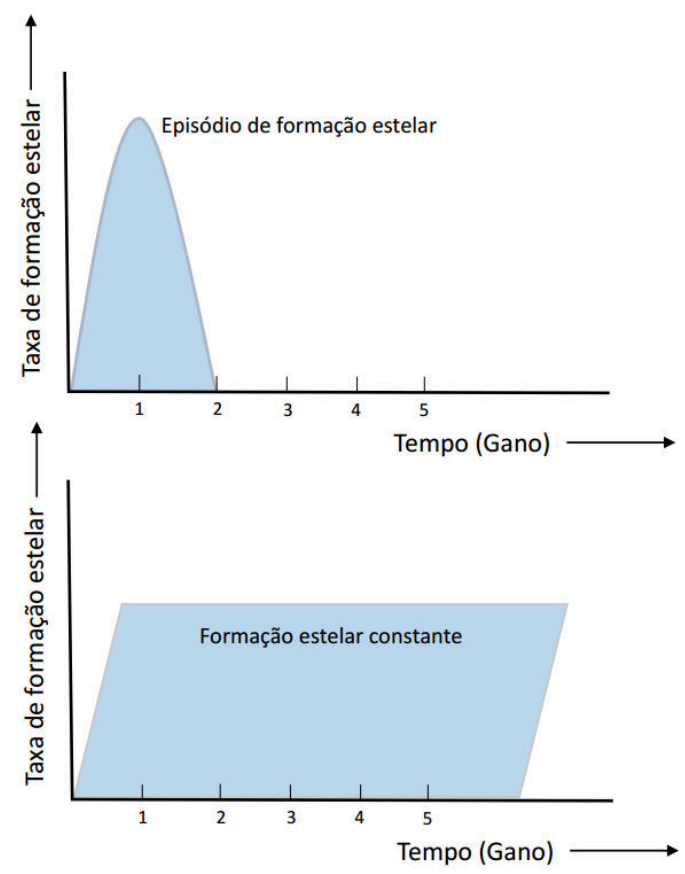

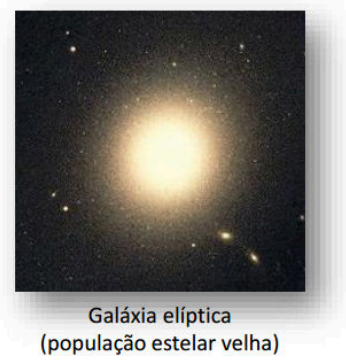

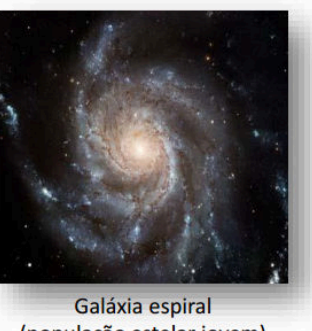

(população estelar jovem)

Figura 1.4: Histórico de formação estelar para galáxias elípticas e espirais. Fonte: Bastos $(2017)$

A diferença na população estelar indica cenários distintos de formação estelar para os dois principais tipos morfológicos, elípticas e espirais. A população mais velha presente em elípticas sugere que essas galáxias tiveram intenso episódio de formação estelar em altos redshifts $(z>2)$ sem uma subsequente formação de estrelas, de maneira que a galáxia apresenta uma evolução passiva. O domínio de população estelar jovem em galáxias espirais, indica que a formação estelar nessas galáxias ocorre de forma constante, ou em diversos episódios. Esse modelo de evolução de elípticas é conhecido como "Modelo de colapso monolítico". Outra forma possível de evolução, é o modelo hierárquico de formação de estruturas, conforme visto anteriormente, as galáxias crescem pela acreção de objetos vizinhos. Por exemplo, a fusão de duas galáxias espirais pode resultar em uma elíptica. Na Figura 1.4 temos um resumo desses cenários de formação estelar.

\subsubsection{Processos internos}

Como vimos anteriormente, a diversidade dos tipos morfológicos observados é fruto dos diferentes processos físicos que ocorrem ao longo da evolução das galáxias. De modo geral, podemos classificar esses processos em dois tipos, internos e externos. Nessa seção discutimos basicamente os processos internos aqueles que têm origem a partir da própria 
galáxia. A discussão dos processos externos é feito na seção 1.4.2, onde descrevemos algumas influências do ambiente sobre a evolução das galáxias.

Feedback é uma das questões ainda em aberto na teoria moderna de formação de galáxias e está ligado com o problema do "resfriamento excessivo". Esse problema foi observado ainda nas primeiras simulações que previam galáxias mais massivas do que as observadas (White e Rees, 1978, Balogh et al., 2001). A razão desse problema era devido ao gás resfriar-se rapidamente gerando uma alta taxa de formação estelar. Uma solução para o problema foi o aquecimento do gás através de explosões de supernovas ou feedback por AGN White e Frenk (1991); Bower et al. (2006). Estudos recentes tem mostrado que a energia liberada por explosões de supernovas não é suficiente para resolver o problema do resfriamento do gás, o que torna o processo de feedback de AGN importante. Quanto ao método de transferência de energia, os mais estudados são: (i) radiativo - o AGN aquece radiativamente o gás frio em torno e (ii) mecânico - jatos de partículas de alta energia removem efetivamente o gás frio da galáxia.

Evolução secular é um processo que ocorre em galáxias disco. A evolução secular pode gerar os chamados "pseudo-bojo", formados pelo rearranjo da estrutura de disco. Estas estruturas são compostos pelo gás do disco que devido a redistribuição do momento angular migra para regiões mais centrais da galáxia. Distinguem-se dos bojos ditos "clássicos" por preservarem características do disco como forma achatada.

\subsubsection{Galáxias de Núcleo Ativo: AGNs}

A maioria das galáxias apresentam um espectro dominado por linhas de absorção, resultado do espectro combinado das estrelas. Entretanto, existem galáxias com fortes linhas de emissão, e que emitem em raios-X. Esta são chamadas galáxias de núcleo ativo, (AGN, do inglês Active Galactic Nuclei). As AGNs possuem um buraco negro supermassivo (BNS) ativo em seu centro. As suas características únicas são devido a essa atividade nuclear.

No paradigma atual, todas as galáxias têm um buraco negro no seu centro Kormendy e Richstone, 1995), e em algum momento é esperado que estas galáxias se tornem ativas. De fato, os buracos negros parecem desempenhar um papel fundamental na formação e evolução de galáxias. Gebhardt et al. (2000); Ferrarese e Merritt (2000) observaram uma correlação forte entre a massa do BNS e a dispersão de velocidades do bojo da galáxia hospedeira. Essa correlação aponta para um cenário onde galáxia e o BNS crescem em 
massa de maneira conjunta.

\subsubsection{Interações Galácticas}

As interações galácticas são processos gravitacionais que acontecem geralmente em pares. Podemos citar por exemplo, interações de maré, fusão galáctica e assédio galáctico. Também existe a fricção dinâmica que é um processo de $\mathrm{N}$ corpos. Esses processos podem alterar significativamente a morfologia das galáxias. Especificamente a fusão galáctica podem gerar um surto de formação estelar, ou engatilhar a atividade nuclear.

\subsubsection{Fusão Galáctica}

Acontece quando duas galáxias se colidem e tornam-se uma só. Geralmente as galáxias que resultam desse encontro apresentam sinais como "caudas" de gás. Entretanto, o encontro de duas galáxias nem sempre gera uma fusão de galáxias. De modo grosseiro, a fusão depende da velocidade relativa dos objetos e da dispersão de velocidades interna da galáxias de maneira que $\sigma_{\text {gal }} \gtrsim v_{\text {colisao }}$. Por exemplo, fusões devem ser raras em aglomerados ricos, pois, a velocidade típica de uma galáxia nesse ambientes é maior que $600 \mathrm{~km} / \mathrm{s}$ enquanto $\sigma_{\text {gal }} \approx 100-300 \mathrm{~km} / \mathrm{s}$.

Quando a fusão galáctica acontece pode ser classificada em dois grande tipos, major merger e minor mergers. No primeiro caso acontece entre galáxias de mesma massa. Toomre (1977) em um trabalho pioneiro de simulações mostrou que a fusão de duas galáxias espirais produz uma galáxia elíptica. Porém, simulações mais recentes, como por exemplo Barnes (2002); Hopkins et al. (2009), mostram um cenário mais abrangente. O tipo morfológico do remanescente de uma fusão depende se as galáxias são ricas ou não em gás em inglês o termo usado é $d r y$ ou wet. No caso de fusão $d r y$ o disco permanece enquanto na fusão wet é produzida uma galáxia tipo jovial. Além do mais, na fusão wet o gás pode iniciar novos episódios de formação estelar e gerar surtos de formação estelar. Isso acontece quando o gás é canalizado na direção do centro da galáxia, onde se resfria e gera o evento. Esse mesmo processo também pode ser responsável por engatilhar a atividade nuclear de AGNs. Sendo assim, esse tipo de fusão pode ser responsável pela alta taxa de formação estelar observadas em galáxias em alto redshift, as famosas galáxias Starburst.

O segundo tipo de fusão, minor mergers, é o mais comum, e envolve uma galáxia até quatro vezes mais massiva do que a outra. Lotz et al. (2011) estima que a taxa de minor 
mergers é três vezes maior do que a taxa de major mergers em $z \sim 0.7$

As fusões afetam a evolução dos buracos negros supermassivos (BNS), pois, devido a fricção dinâmica, os dois BNS perdem energia orbital, se aproximam e eventualmente se coalescem e emitem ondas gravitacionais. Também pode acontecer de BNS escapar e parar no meio intergaláctico.

Em suma, fusões de galáxias podem em principio transformar a morfologia de galáxias disco em tipo joviais. Também são capazes de gerarem surtos de formação estelar e disparar a atividade de AGNs. Na formação hierárquica de estruturas, essas interações possuem um papel relevante na formação e evolução de galáxias.

\subsubsection{Assédio Galáctico}

Em aglomerados de galáxias, a velocidade típica de um membro é por volta da dispersão de velocidade do próprio aglomerado - na região em que há equilíbrio gravitacional do sistema. Esta dispersão de velocidades é muito maior que a dispersão de velocidade interna das galáxias, conforme explicado na seção anterior mesmo no encontro de duas galáxias não haverá fusão. De modo que, as interações galácticas em aglomerados são do tipo de alta velocidade. Com exceção das regiões mais externas, em que as galáxias não seguem necessariamente a distribuição de velocidades das regiões virializadas - i.é. não seguem o teorema do virial.

No encontro de alta velocidade não há coalescência, o que acontece é uma perturbação do potencial, podendo gerar mudanças estruturais. O efeito acumulativo de vários encontros à alta velocidade é denominado "Assédio Galáctico".

Simulações realizadas por Moore et al. (1996, 1998) mostram que galáxias disco que sofrem diversos encontros com membros massivos, podem perder grande parte de sua massa, pois, o aquecimento provocado pelo o aumento da energia interna pode levar as estrelas de órbitas não ligadas. As estrelas que permanecem no disco ganham energia cinética e geram uma transformação estrutural do disco. Esse processo deve ser efetivo em aglomerados. 


\subsection{Efeitos dos Aglomerados na Evolução de Galáxias}

Nessa seção fazemos uma breve introdução de efeitos ambientais que afetam a evolução de galáxias. Descrevemos alguns dos mecanismos e processos físicos que alteram significativamente o seu destino, e por último, introduzimos as principais evidências observacionais dessas relações em aglomerados.

As galáxias sofrem alterações em diferentes tipos de ambiente, nesse trabalho vamos falar principalmente de efeitos e processos que são comuns em aglomerados ou em pares de galáxias. Podemos resumir esses efeitos em: segregação morfológica, sequência vermelha, efeito Butcher-Oemler, perda de gás das espirais, interações entre galáxias e galáxias centrais gigantes $(\mathrm{cD})$. Vamos comentar sobre os efeitos que são relevantes a esse trabalho na seção 1.4.2. Mas antes disso, revisamos um mecanismo importante na evolução de galáxias em aglomerados, a pressão de arraste.

\subsubsection{Pressão de Arraste}

A pressão do gás intra-aglomerado (ICM) é geralmente maior que a pressão do gás neutro, HI, nas galáxias espirais. Quando essas galáxias caem no aglomerados, pode vir a acontecer de perderem o gás do meio interestelar, essencialmente HI, pelo efeito de pressão de arraste ou ram-pressure. Na Figura 1.5 temos uma ilustração desse efeito sobre uma galáxia espiral em queda no aglomerado.

De forma quantitativa, a pressão de arraste do ICM depende da sua densidade, $\rho_{I C M}$ e da velocidade relativa da galáxia, $v_{v}$, da seguinte forma:

$$
P_{\text {ram }}=\rho_{I C M} v^{2}
$$

A remoção do gás ocorre quando a pressão de arraste é maior que força gravitacional por unidade de área que mantém o gás do ISM. Sarazin (1986) considerando uma galáxia disco caindo no aglomerado de diâmetro, $2 r_{\text {disco }}$, massa, $M_{\text {disco }}$, densidade superficial uniforme e supondo que $v^{2}=3 \sigma_{\text {cls }}^{2}$ escreveu essa condição da seguinte forma:

$$
\left(\frac{n_{I C M}}{10^{-3} \mathrm{~cm}^{-3}}\right)\left(\frac{\sigma_{\text {cls }}}{10^{3} \mathrm{~km} / \mathrm{s}}\right)^{2} \gtrsim 3\left(\frac{M_{\text {disco }}}{10^{11} M_{\odot}}\right)^{2}\left(\frac{r_{\text {disco }}}{10 k p c}\right)^{-4}\left(\frac{M_{I S M}}{10^{-1} M_{\text {disco }}}\right)
$$

onde $n_{I C M}$ é a densidade numérica de átomos no ICM e $M_{I S M}$ é a massa do ISM. Por uma lado as galáxias de maior diâmetro ou de menor massa têm maior probabilidade de 


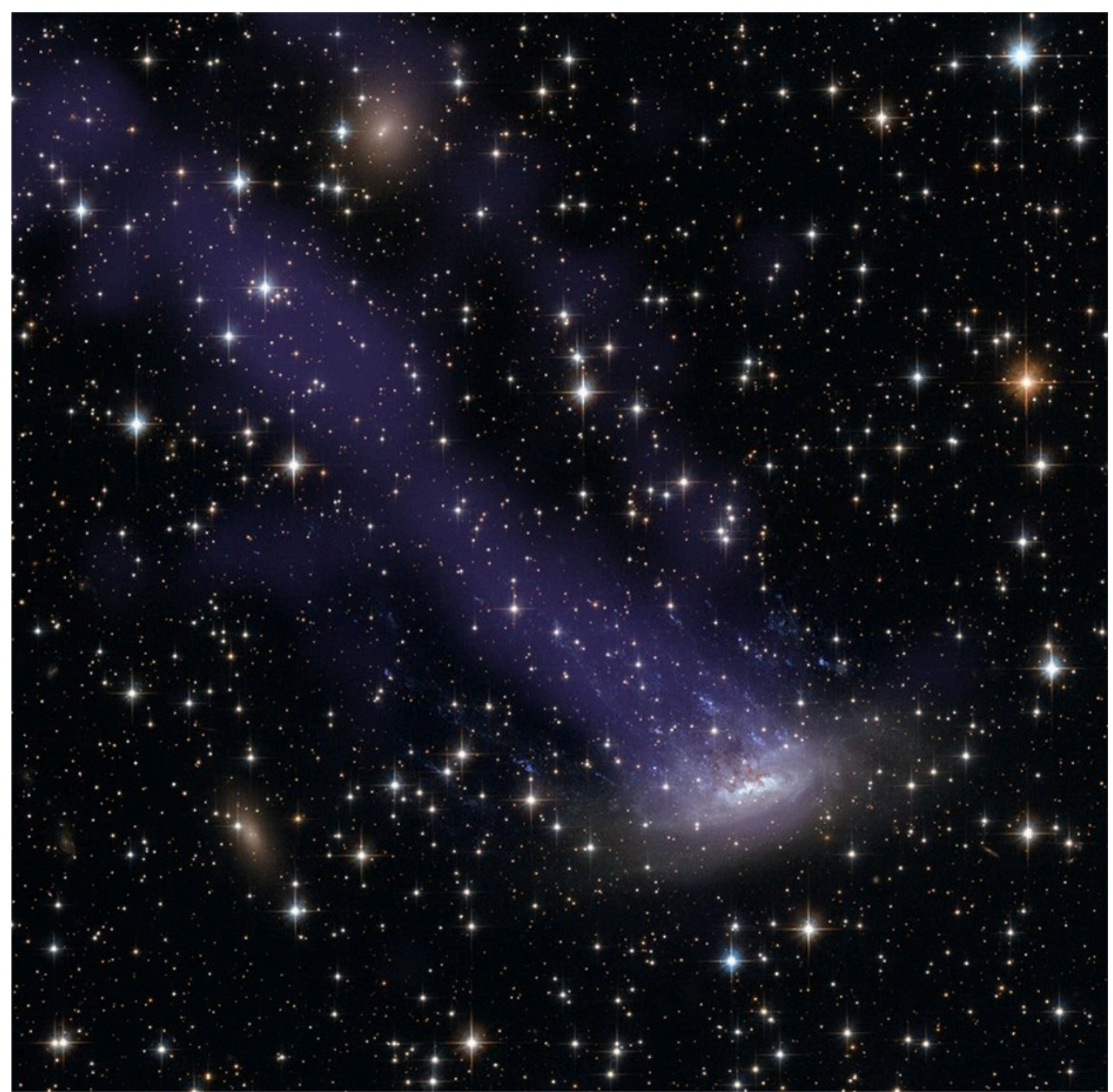

Figura 1.5: A galáxia ESO 137-001 do tipo tardio caindo no aglomerado de galáxias Abell 3627. O meio intraglomerado (ICM) atua como um vento no meio interestelar (ISM) da galáxia, e o arranca para fora através da remoção por pressão de arraste. A cauda de $\mathrm{H}-\alpha$ e raios- $\mathrm{X}$ deixada para trás tem por volta de $80 \mathrm{kpc}$ de comprimento. A imagem é composta no óptico e em raios-X, por observações do HST e Chandra, respectivamente. Créditos: Raios-X: NASA/CXC/UAH/M.Sun et al; Optico: NASA, ESA, \& the Hubble Heritage Team (STScI/AURA)

terem seu gás removido de um aglomerado massivo (maior $\sigma_{c l s}$ ). Por outro lado, esse processo deve ser eficiente em aglomerados mais massivos e em regiões próximas ao centro do aglomerado, pois, $n_{I C M}$ é tipicamente da ordem de $10^{-2}-10^{3} \mathrm{~cm}^{-3}$ no centro e decresce fortemente em regiões além do raio do virial.

Rugiero \& Lima Neto (2018) usando simulações hidrodinâmicas mostraram que uma galáxia espiral tipo Via Láctea caindo em um sistema cool-core (CC) perde todo seu gás ao alcançar o centro do aglomerado. O resultado é independente das formas de entrada da galáxia e da massa do aglomerado no regime entre $10^{14} M_{\odot}$ e $10^{15} M_{\odot}$. O mesmo não acontece para um sistema não CC, a galáxia perde apenas parte do seu gás nesse caso, 
e a quantidade de gás remanescente depende da massa do aglomerado. Antes da galáxia atingir o centro, constataram um aumento a taxa de formação estelar em torno de 1.5-3.

Do ponto de vista evolutivo, quando uma galáxia perde grande parte do gás interestelar, ela também perde o ingrediente fundamental para formação de novas estrelas. Desse modo, a formação estelar é extinta. O processo de pressão de arraste em si não é capaz de alterar a morfologia da galáxia porém, como sua principal consequência é reduzir o gás disponível, uma galáxia de disco pode se transformar em uma lenticular S0 ou uma espiral passiva dado o fim da formação estelar.

\subsubsection{Estrangulamento}

Quando uma galáxia que está sendo adicionada a um meio de alta densidade pode perder o seu reservatório de gás, processo chamado de Estrangulamento. Nesse caso não há interrupção abrupta da formação estelar. Ao perder o reservatório, a galáxia continua a formar estrelas no disco até exaurir o seu gás. Na Figura 1.6 apresentamos as principais diferenças entre pressão de arraste e estrangulamento.

\subsubsection{Qual a influência do ambiente na evolução de galáxias?}

Nas seções anteriores vimos os principais processos que são responsáveis pela transformação da morfologia de galáxias. De modo que cada processo necessita de condições ideais regidas pelo meio. Galáxias pertencentes a aglomerados são mais propícias a sofrer processos como Assédio Galáctico. Pressão de arraste e estrangulamento são processos que podem acontecer com galáxias que caem no aglomerado, e são mais eficientes perto do centro. Seriam as interações favorecidas em um dado ambiente o processo dominante na evolução de galáxias? Ou seriam as propriedades intrínsecas iniciais que determinam sua evolução? Essas questões são amplamente discutidas na literatura e são conhecidas como o debate Nature vs Nurture.

Um dos fenômenos de ambiente denso mais evidente é a segregação morfológica, notada desde os primórdios da Astronomia Extragaláctica por Hubble e Humason (1931). Dressler (1980) mostrou que os tipos morfológicos de galaxias tipo joviais, E+S0, constituem 90\% da população galáctica em ambientes mais densos enquanto em regiões de campo sua frequência é por volta de 40\%, usando uma amostra de 55 aglomerados do universo local. Estudos posteriores em redshifts mais altos, Dressler et al. (1997); Smith et al. (2005), 
(a)
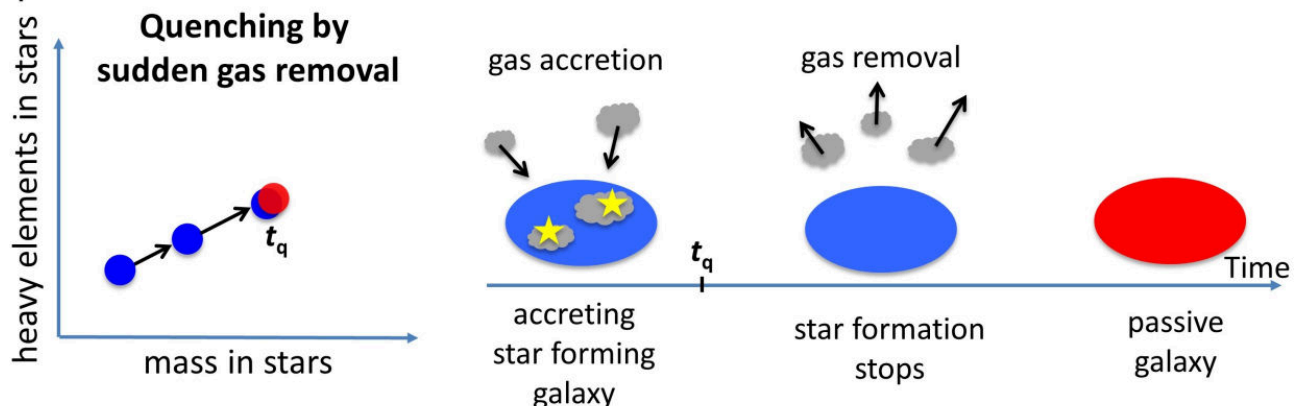

(b)
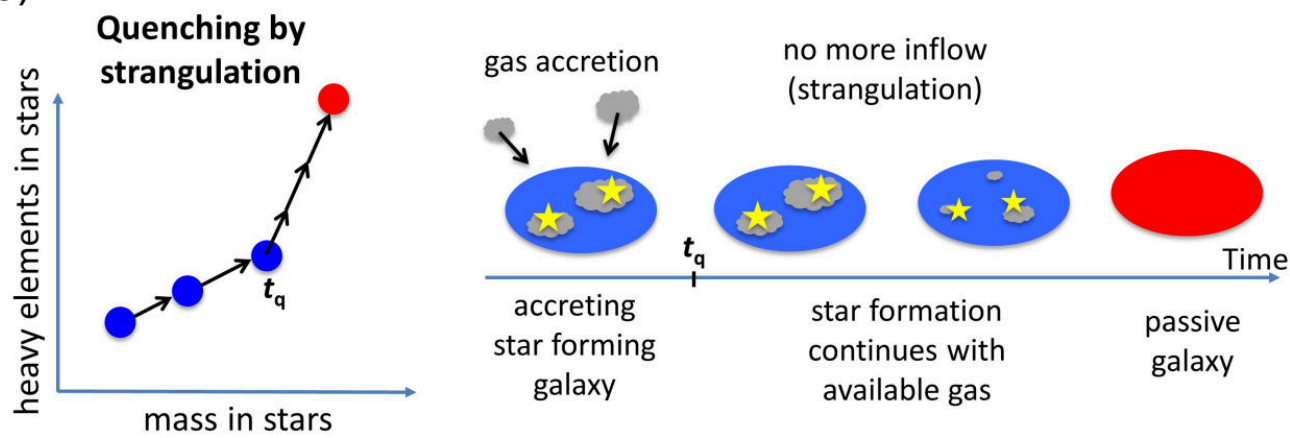

Figura 1.6: Processos de pressão de arraste (a) e estrangulamento em galáxias (b). Em (a) a remoção do gás acontece de forma rápida, em $t=t_{q}$, resultando em uma evolução passiva. Em (b) a galáxia perde seu reservatório de gás ao ser adicionada em um ambiente de alta densidade em $t=t_{q}$. A formação estelar não é interrompida e se mantêm até que todo o conteúdo de gás presente na galáxia se esgote. Fonte Peng et al. (2015)

mostraram que a relação ainda é presente. Porém, é menos intensa, a fração de E+S0 diminui, tomando valores em torno de $85 \%$ entre $0.35<z<0.57$ e $70 \%$ entre $0.75<z<$ 1.27, como mostra a Figura 1.7. A origem da segregação morfológica pode estar ligada ao chamado efeito Butcher \& Oemler.

Uma consequência do ambiente sobre a cor da galáxias é a sequência vermelha, que é uma relação cor-magnitude encontrada em aglomerados. Esta relação é composta majoritariamente por galáxias elípticas e S0. Do ponto de vista técnico, a sequência vermelha é usada para identificar aglomerados (Gladders e Yee, 2000; Rykoff et al., 2014). A natureza dessa relação pode estar ligada a um efeito de idade, ou um efeito da metalicidade. Sendo que as galáxias mais brilhantes são mais ricas em metais, pois, os sistemas mais massivos são capazes de reter de maneira mais eficaz os metais produzidos e ejetados por estrelas de maior massa, de maneira que os ventos galácticos produzidos por supernovas não são fortes o bastantes para expulsar os metais do meio intergaláctico. Esta hipótese é mais provável e suportada por uma série de trabalhos que indicam a existência de uma relação 

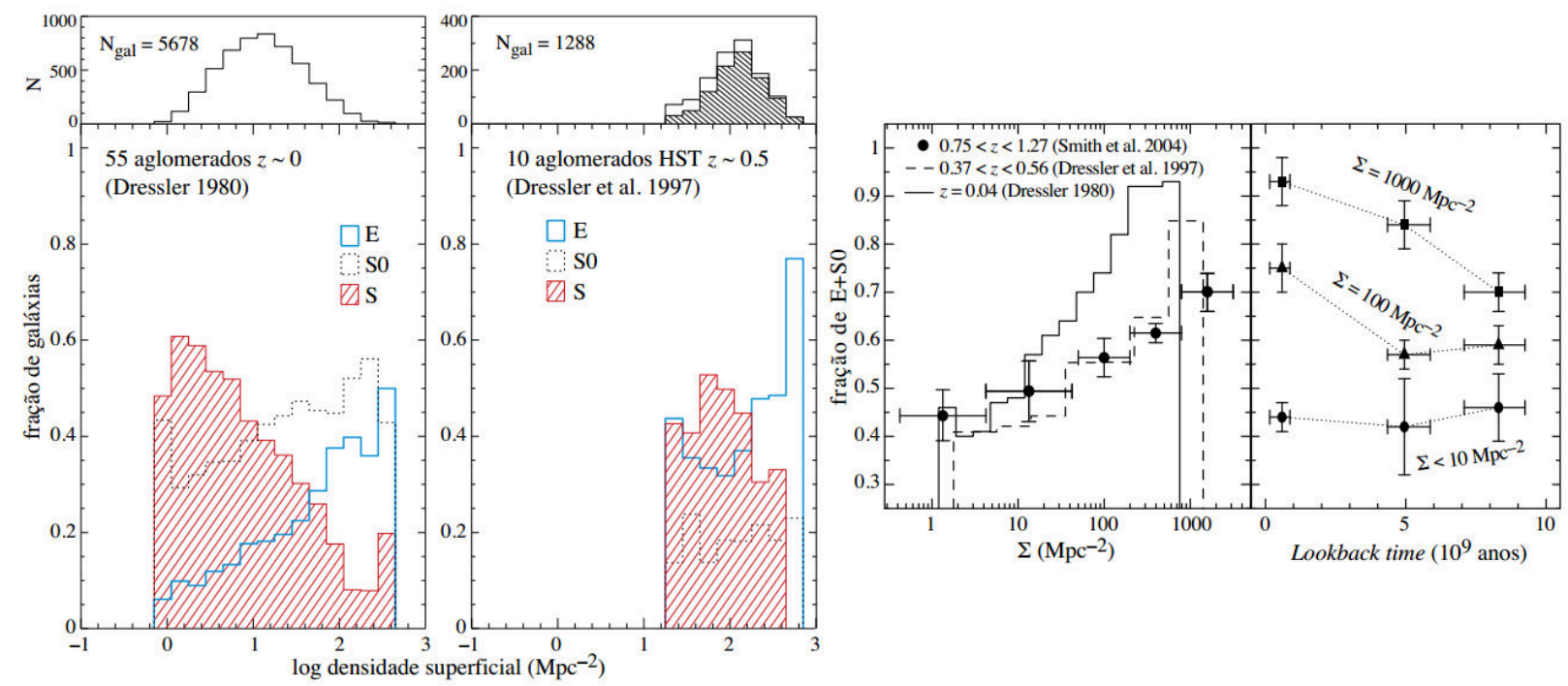

Figura 1.7: Relação entre o tipo morfológico e a densidade local para galáxias em aglomerados. O histograma no alto de cada painel mostra o número de galáxias para cada intervalo de densidade superficial. Para os aglomerados do HST $(0.36<z<0.57)$, o histograma hachurado do alto mostra a distribuição de galáxias após correção da contaminação de galáxias na linha de visada. Os dois gráficos da esquerda são dados de Dressler (1980); Dressler et al. (1997) e da direita são de Smith et al. (2005) com dados adicionais até $z \approx 1$. Fonte: Lima Neto $(2018)$

luminosidade-metalicidade (Ellis et al., 1997; Ferreras et al., 1999; Terlevich et al., 1999). Entretanto, alguns modelos semi-analíticos de formação e evolução apontam para uma explicação mista, a relação cor-magnitude seria devido a um efeito de idade e metalicidade, ou seja, as galáxias mais massivas são também as mais antigas.

A primeira evidência de evolução de galáxias em ambiente densos foi encontrada por Butcher e Oemler (1978, 1984) quando observou um aumento gradual da fração de galáxias azuis no intervalo $0.1<z<0.4$. Posteriormente foi confirmado fotometricamente por uma série de estudos (p. ex.: Rakos e Schombert, 1995; Margoniner e de Carvalho, 2000; Goto et al., 2003). Como vimos na seção 1.3.2, galáxias azuis são relacionadas a formação estelar recente pois as estrelas mais jovens são quentes e azuis. Os estudos utilizando a base de dados 2dF Galaxy Redshift Survey (Lewis et al., 2002) e SDSS (Gómez et al., 2003) mostram que a taxa de formação estelar decresce para galáxias em ambientes de alta densidade e que tal fenômeno ocorre em todos os regimes de densidade e redshift. A pressão de arraste juntamente com estrangulamento são mecanismos importantes responsáveis por esse decréscimo. Interações galácticas também podem ter um papel importante, em particular o assédio galáctico. 


\subsubsection{AGNs em Aglomerados}

O meio ambiente não afeta apenas a morfologia e taxa de formação estelar, mas também afeta a atividade nuclear em galáxias, ou seja, tem um impacto no fenômeno de núcleos ativos de galáxias, AGNs. O estudo de AGNs é crucial para entendermos os mecanismos que disparam esta atividade.

A ligação entre a atividade de AGNs e o meio ambiente foi revelado há 4 décadas (Gisler, 1978; Dressler et al., 1985), quando notaram a ausência de galáxias com linhas de emissão em aglomerados, em relação ao campo. Com efeito, AGNs em aglomerados são mais raras em comparação com o campo. Apesar da dependência de AGNs ser afetada pelo ambiente ainda ser debatida, vários autores defendem essa tese (p. ex.: Kauffmann et al., 2004; Gilmour et al., 2007; Ehlert et al., 2015; Lopes et al., 2017; Gordon et al., 2018), alguns com menos afinco, pois possuem evidências marginais para tal conexão (Man et al., 2019), enquanto outros estudos que fizeram a seleção de AGNs em raios-X discordam (Haggard et al., 2010; Koulouridis et al., 2014).

O mecanismo por trás que dispara a atividade de buracos negros supermassivos (BNS), consequentemente AGNs, ainda não está satisfatoriamente entendido. O abastecimento da atividade nuclear depende do reservatório de gás frio da galáxia e da eficiência do mecanismo de transporte do gás até a região central, onde o BNS se localiza. Qualquer mecanismo que exerça um torque sobre o gás em direção central pode em principio gerar a atividade nuclear. Acredita-se que instabilidades de barra (Hopkins et al., 2008) e interações de galáxias são mecanismos dominantes, e recentemente, a pressão de arraste (Marshall et al., 2018) no contexto de aglomerados também foi discutida como um possível mecanismo.

Nesse sentido, esta dissertação tem por objetivo estudar a dependência de AGNs com o meio de aglomerados. E explorar as causas possíveis para dessa possível dependência. Para tal investigação, utilizamos observações em raios-X e no óptico em um largo intervalo de redshift $0.1<z<0.65$. Contamos com propriedades importantes do meio, como massa, temperatura e estado dinâmico. Buscamos correlacionar essas informações com a demografia e fração das AGNs e também estudá-las ao longo do redshift. 


\section{Base de dados}

Nesta seção descrevemos o conjunto de dados adotados nesse estudo, que usa a força da Astronomia Multibandas. Utilizamos dados no óptico dos dois maiores levantamentos fotométricos existentes, SDSS e DES. Em raios-X, usamos a PSF 1 única do telescópio Chandra - propriedade imprescindível na identificação das AGNs.

\subsection{Astronomia em Raios-X: Chandra}

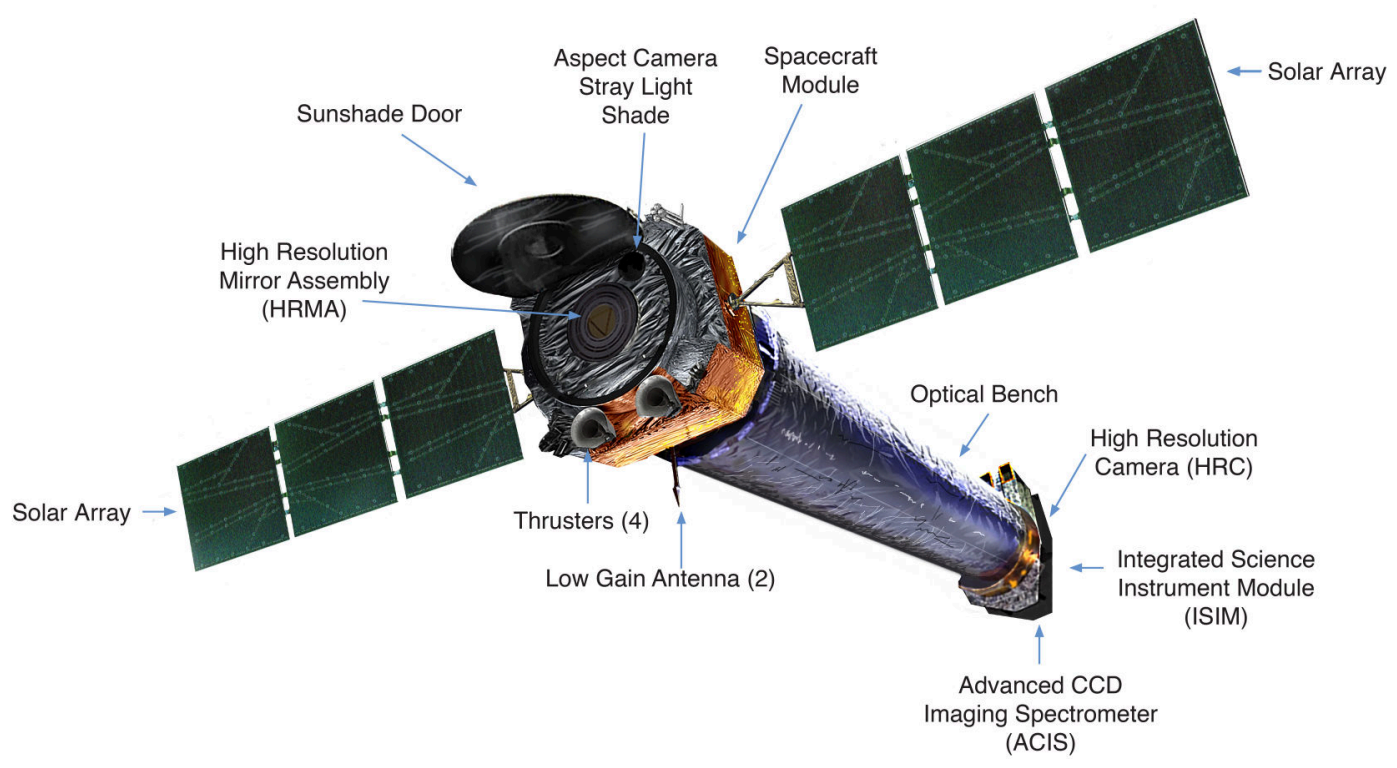

Figura 2.1: Satélite Chandra - Visão panorâmica dos instrumentos. Fonte: http://chandra.harvard. edu/about/spacecraft.html

O telescópio espacial Chandra lançado em julho de 1999, carrega esse nome em homenagem ao astrofísico Subrahmanyan Chandrasekhar. Na Figura 2.1 temos uma ilustração

\footnotetext{
${ }^{1}$ (do inglês, Point Spread Function)
} 
da espaçonave. Esse satélite está em orbita até os dias de hoje, e possuí uma orbita elíptica com um apogeu de $133.000 \mathrm{~km}$ e perigeu de $16.000 \mathrm{~km}$. O seu período orbital é cerca de 2 dias e 16 horas, permitindo assim observações de aproximadamente $190 \mathrm{ks}$ sem intervalos. Se sua orbita é vantajosa por um lado, tem sua desvantagem por outro, o satélite por mais de $80 \%$ do tempo está acima do cinturão de Van Allen, i.e., está desprotegido do bombardeio de partículas energéticas emitidas pelo Sol. Explosões solares que aumentam a taxa de raios cósmicos no vento solar (os flares) atingem o satélite e formam o chamado fundo de partículas (particle background). Observações são por vezes perdidas devido aos flares.

Do ponto de vista técnico, o Chandra possuí um dos detectores mais sensíveis em raios$\mathrm{X}$ e sua característica preciosa é sua resolução espacial da ordem de um segundo de arco comparável a resolução no óptico em telescópios clássicos na Terra. O seu campo de visão é de $16 \times 16 \operatorname{arcmin}^{2}$. Nesse trabalho, usamos o arranjo de detectores ACIS-I (Advanced CCD Imaging Spectrometer), conforme ilustrado na Figura 2.2 .
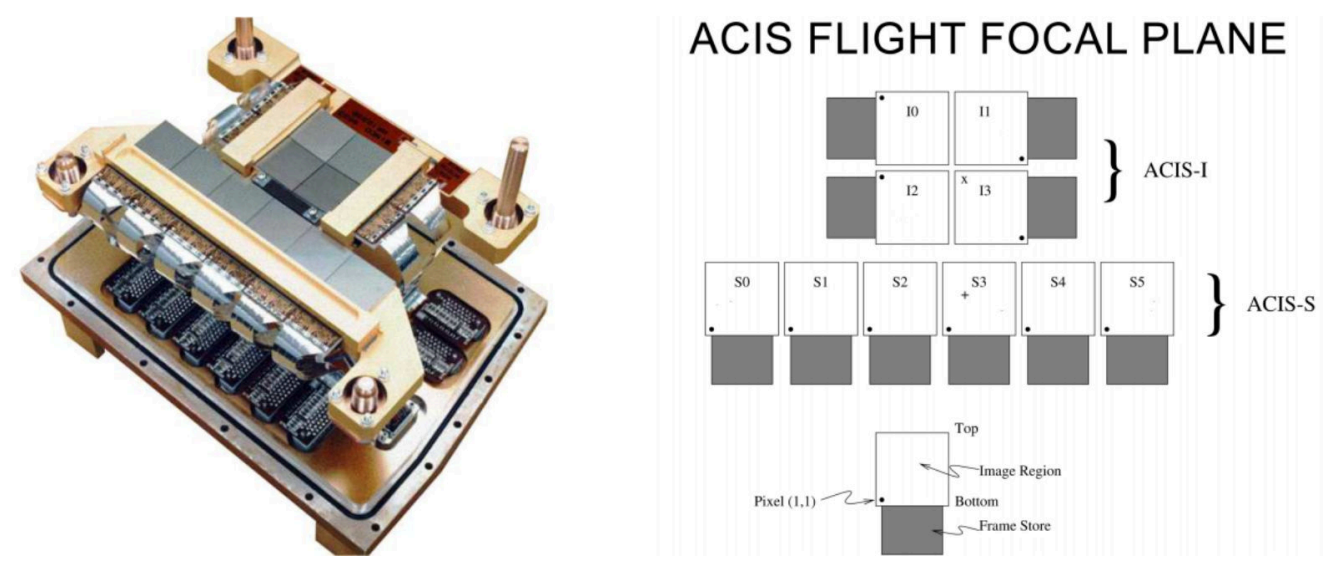

Figura 2.2: Esquema do arranjo de detectores ACIS. O arranjo dos 4 chips de ACIS-I tem um campo de visão por volta de $16 \times 16 \operatorname{arcmin}^{2}$ e $1024^{2}$ pixeis. Os chips S1 e S3 usam o sistema chamado backilluminated, o que os torna mais sensíveis; os outros chips são front-illuminated. Fonte: Andrade-Santos (2008)

\subsection{Amostras}

Nesta seção descrevemos o conjunto de observações em raios-X que compõe esse trabalho.

Maughan et al. (2008) estimou os parâmetros físicos de um conjunto de aglomerados e também caracterizou as propriedades do estado dinâmico desses objetos. O catálogo foi 
produzido a partir de observações do arquivo público com alta resolução (até novembro de 2006) do satélite Chandra e contém 115 aglomerados de galáxias observados com o detector ACIS-I no intervalo de redshift $0.1<z<1.3$. O limite inferior em redshift, assegura que toda a emissão dentro do raio $R_{500}$ do aglomerado pode ser observada no campo de visão do satélite Chandra (raio onde a densidade é 500 vezes a densidade crítica do universo).

Os aglomerados da amostra são de uma coletânea heterogênea, i.e. foram observados para diferentes propósitos. Quase a metade dos objetos (55) foram observados pelo tempo destinado a Leon Van Speybroeck como parte de um programa para combinar observações em raios-X e em SZE para colocar vínculos nos parâmetros cosmológicos $H_{0}$ e $\Omega_{M}$ (Bonamente et al., 2006). Apesar de ter mais de uma década, não há ainda outro catálogo disponível com essas características.

Em 2012, a amostra teve suas medidas refeitas e aprimoradas (Maughan et al., 2012, M12, daqui em diante). A propagação de erro foi aprimorada nas medidas de $R_{500}$ e de desvio do centro $\langle w\rangle$. Houve uma mudança nos valores estimados de temperatura de alguns aglomerados, essa diferença foi devido a limpeza de flares. Por fim, o redshift do aglomerado AS1063 foi corrigido. Utilizamos essa versão em nosso trabalho.

XMATCHA é uma compilação de todos aglomerados do catálogo do redMaPPer v6.4 que possuem observações em raios-X feitas pelo Chandra (Hollowood et al., 2018). Esse catálogo tem dados em raios-X com uma taxa de contagens miníma para fazer um ajuste de temperatura em uma abertura de $500 \mathrm{kpc}$. No entanto, esse catálogo possuí apenas medidas de temperatura.

Nesse trabalho utilizamos a amostra de objetos que está no campo de observação do DES Y1 (Palmese et al., 2019). O intervalo de redshift é de 0.1 até 0.7. Aplicamos a nossa própria metodologia para estimar as propriedades físicas em raios-X - i.e. centro, temperatura, massa, massa do gás e parâmetros do estado dinâmico.

\subsection{Chandra Source Catalog (CSC)}

As fontes pontuais em raios-X tem duas origens basicamente, AGNs e binárias cataclísmicas.

O catálogo de fontes pontuais do Chandra (CSC, do inglês, Chandra Source Catalog ${ }^{2}$ )

\footnotetext{
${ }^{2}$ CSC homepage - http://cxc.cfa.harvard.edu/csc1/
} 
tem como objetivo fornecer acesso às melhores estimativas das propriedades da fonte de raios-X para fontes detectadas e facilitar a análise de uma ampla gama de propriedades estatísticas para classes de fontes de raios-X.

O catálogo inclui fontes de raios-X detectadas com estimativas de fluxo que são pelo menos 3 vezes suas incertezas $(\sigma)$ em pelo menos uma banda de energia. A primeira versão CSC 1.0 inclui informações sobre 94.676 fontes de raios-X detectadas com observações feitas pelo instrumento ACIS no período do início das das observações até 2009 (Evans et al. 2010). O conteúdo da versão 2.0 foi parcialmente disponibilizada, com cerca de $99 \%$ das observações analisadas e 315.880 fontes confirmadas.

O acesso ao catálogo é feito pelo software CSCview. Este programa tem opções de fazer buscas por nome, por coordenadas e por uma lista de objetos. Os dados de saída são inúmeros, desde informações da observação até propriedades espectrais da fonte. Para o nosso trabalho, as propriedades mais relevantes são a posição, o fluxo de energia na banda broad (0.5-7.0 keV), e o hardness-ratio entre as bandas hard (2.0-7.0 keV) e soft (0.5-2.0 keV). Na Figura 2.3 temos um exemplo de busca.

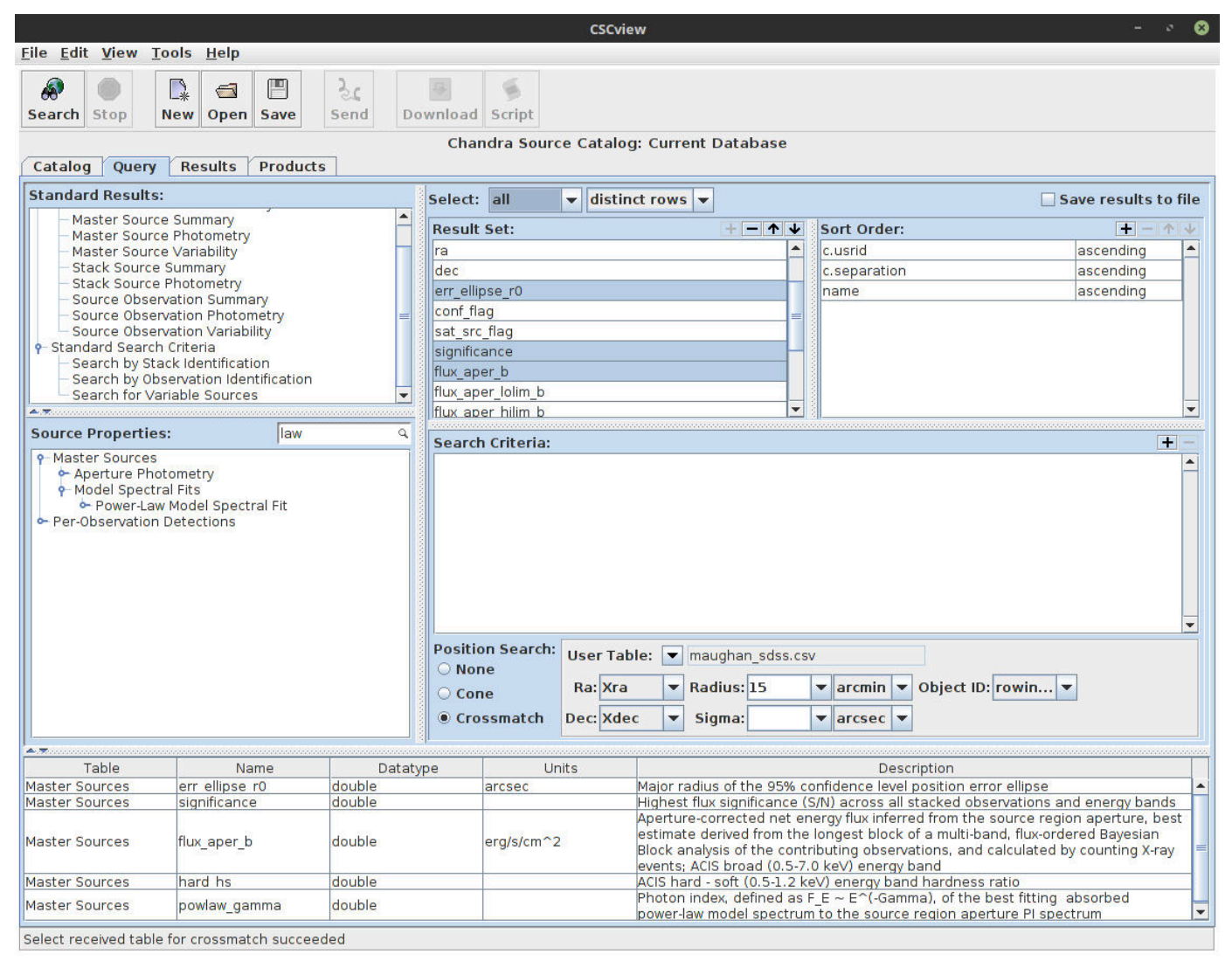

Figura 2.3: Interface do CSCview 
Fontes pontuais são por definição objetos com tamanho angular menor que a precisão do detector, ou seja, a PSF. Geralmente os detectores ópticos possuem PSFs bem conhecidas e precisas, porém o mesmo não acontece em raios-X em geral, salvo os detectores do Chandra que tem precisão média de $0.5^{\prime \prime}$ a $1^{\prime \prime}$. No entanto, essa varia com a energia e com o eixo óptico do telescópio. Por exemplo, para uma detecção perto do eixo, a PSF é de apenas $0.3^{\prime \prime}$, para deteç̧ões a $15^{\prime}$ do eixo, a escala é da ordem de $13^{\prime}$, isso na banda de energia de $1.5 \mathrm{keV}$. Porém, essas deteç̧ões fora do eixo óptico $\left(>15^{\prime}\right)$ não são típicas (Evans et al., 2010). Na Figura 2.4 mostramos a imagem de um fonte pontual simulada para detecções do Chandra em 3 ângulos em relação ao eixo óptico.

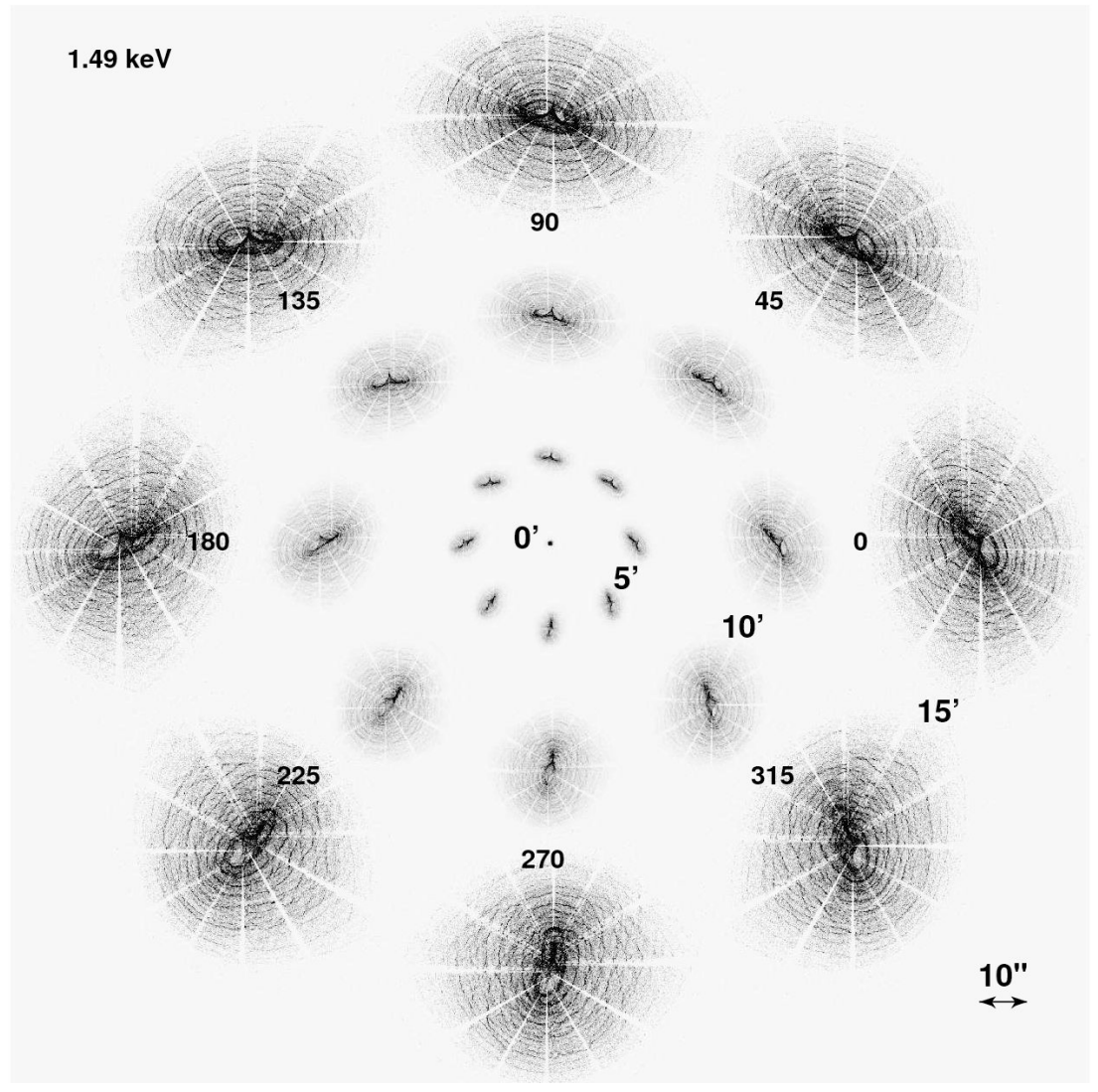

Figura 2.4: Imagens simuladas em $1.49 \mathrm{keV}$ de detecções do Chandra-HRMA. As fontes são mostradas como apareceriam no céu, em três ângulos em relação ao eixo óptico $\left(5^{\prime}, 10^{\prime}\right.$ e $\left.15^{\prime}\right)$ e vários azimutes. Fonte:http://cxc.harvard.edu/proposer/POG/html/chap4.html

Um ponto importante é a precisão da posição, para a fazer a correlação das fontes pontuais em raios-X com as galáxias é necessário uma precisão de no máximo alguns segundos de arco. Nesse sentido para garantir essa precisão fizemos dois cortes: fontes pontuais com erro na posição menor que 4"; e detecções que estejam abaixo $10^{\prime}$ em relação ao eixo do telescópio. Cerca de $95 \%$ das fontes do nosso catálogo cumprem esses requisitos. 


\subsection{Grandes Levantamentos Fotométricos: SDSS e DES}

Nesta seção introduzimos a base de dados fotométricas e sus características.

\subsubsection{Dark Energy Survey - DES}

O Dark Energy Survey (DES ${ }^{3}$ é uma colaboração internacional, um esforço colaborativo para mapear milhões de galáxias, detectar milhares de supernova e encontrar padrões da estrutura cósmica que buscam revelar a natureza da misteriosa energia escura que está acelerando a expansão do Universo.

Ao longo de 6 anos, foram 575 noites de observação para fazer esse levantamento profundo de uma grande área do céu do hemisfério Sul, cerca de 5000 graus quadrados, que catalogou informações de 300 milhões de galáxias, em 5 bandas fotométricas grizY. Foi utilizada a camera CCD DECam (veja Flaugher et al. 2015) montada no telescópio de 4 metros Blanco localizado no CTIO (do inglês, Cerro Tololo Inter-American Observatory) no Chile. A DECam cobre 3 graus quadrados e tem 570 Megapixels distribuídos em 74 CCDs arranjados em um formato hexagonal.

Usamos neste trabalho os dados do primeiro ano de observações (Setembro de 2013 Fevereiro de 2014, The Dark Energy Survey Collaboration 2014) que cobriu uma região de 1,839 $\mathrm{deg}^{2}$ e com até 4 passagens por filtro. O acesso dos dados é livre e está disponível no endereço http://des.ncsa.illinois.edu/releases/y1a1. Embora usamos a região do DES Y1 (do inglês, year one), a fotometria é provinda de dados mais profundos, o catálogo DES Y3, do ano 3 - tendo uma profundidade de $10 \sigma$ mediana(o)s no catálogo co-adicionado para uma abertura de diâmetro 1.95": $g=24.33, r=24.08, i=23.44$, $z=22.69$, e $Y=21.44$ mag; The Dark Energy Survey Collaboration 2018. Essa fotometria é o resultado do algorítimo Multi-Object Fitting (MOF) que usa o código ngmix $4^{4}$. Os photo$z$ 's foram calculados usando os templates modelo do algorítimo BPZ (do inglês, Bayesian Photometric Redshifts) Benítez (2000).

O catálogo de galáxias usados aqui foi o mesmo usado em (Palmese et al., 2019). Extraímos todas galáxias dentro de um raio de $12 \mathrm{Mpc}$ do centro do aglomerado. E evitamos objetos com cores sem sentido, $(g-r)>4$ e $(g-r)<-1$. Foi usado a magnitude limite na banda $r$ de 24.08 mag do DES.

\footnotetext{
3 www . darkenergysurvey . org

4 https://github.com/esheldon/ngmix
} 


\subsubsection{SDSS}

O Sloan Digital Sky Survey (York e SDSS Collaboration, 2000) é um dos maiores surveys ópticos feitos na superfície da Terra. O levantamento produziu um mapa detalhado de aproximadamente um quarto do céu, contendo imagens de milhões de objetos e quase um milhão de espectros de galáxias. O telescópio usado pelo SDSS, de $2.5 \mathrm{~m}$ de diâmetro, se encontra a $2788 \mathrm{~m}$ sobre o nível do mar, no Apache Point Observatory (APO) em Sunspot, New Mexico, EUA. Possui uma câmera imageadora de campo amplo, abarcando o óptico e o infra-vermelho próximo com cinco bandas fotométricas, $u, g, r, i, z$.

O acesso aos dados do SDSS foi feito pela plataforma CasJobs. Abaixo descrevemos como feito foi seu acceso. A verificação dos objetos que estão no campo do SDSS é feita pela seguinte query:

SELECT dbo.fInFootprintEq(t.RA, t.DEC, 0.1)

into mydb.MyTable from MyDB.MyTable_Chandra AS $t$

A função $f$ InFootprintEq (ra [degrees], dec [degrees], radius [arcmin]) tem como entrada a coordenada do objeto e o raio de verificação.

Para cada aglomerado foi criado uma amostra com galáxias dentro de um círculo de $12 \mathrm{Mpc}$ ao redor do seu centro. A query utilizado é mostrado abaixo.

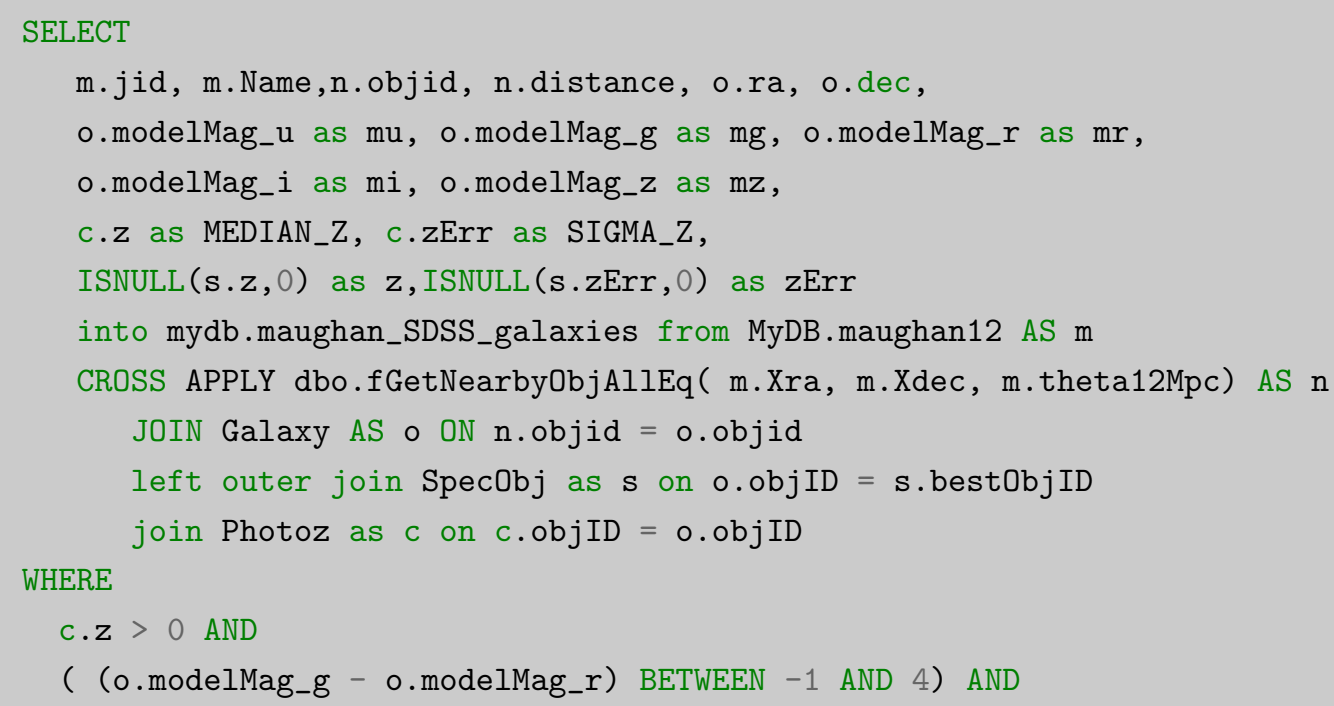

Os dados de saída são as coordenadas (ra,dec), as magnitudes nas 5 bandas, o redshift fotométrico, e quando disponível o redshift. Foi usado a magnitude limite na banda $r$ 
de 22.2 mag do SDSS para garantir a completeza de $95 \%$ das fontes pontuais. Também evitamos objetos com cores sem sentido, $(g-r)>4$ e $(g-r)<-1$.

\subsection{Propriedades da Amostra}

Nesta seção apresentamos as propriedades da amostra de aglomerados usadas nesse trabalho.

\subsubsection{Catálogo de Aglomerados}

Usamos as duas amostras em raios-X com dados fotométricos distintos, isto é, foram usados os dados do SDSS para a amostra M12 e os dados do DES para a amostra XMATCHA. Sendo que a amostra M12 contém 72 aglomerados no campo do SDSS porém, restringimos a amostra para $z<0.35$ devido à pouca profundidade do levantamento. Por fim, utilizamos um total de 36 objetos de M12(SDSS). Em relação à XMATCHA, dos 69 objetos utilizamos as observações que possuíam contagens em raios-X suficiente para ajustar o perfil radial de brilho superficial. Na Tabela 2.1 resumimos as amostras usadas nesse trabalho.

Tabela 2.1 - Amostra em raios-X e no óptico.

\begin{tabular}{l|c|c}
\hline & SDSS & DES \\
\hline Maughan et al. 2012 & $72(36)$ & - \\
XMATCHA & - & $69(61)$ \\
\hline \multicolumn{2}{c}{ entre parênteses temos o número de aglomerados usado } \\
nesse trabalho e sem parênteses temos o número total de \\
aglomerados da amostra em raios-X no campo do levan- \\
tamento fotométrico.
\end{tabular}

Em suma, a nossa amostra final conta com 96 aglomerados distribuídos entre $0.093<$ $z<0.657$. A distribuição de redshift, massa e temperatura dos objetos é mostrada na Figura 2.5. Sendo que estimamos as propriedades física do ICM para a amostra XMATCHA e para a amostra M12 utilizamos os dados já estimados anteriormente. Em particular, o estado dinâmico dos aglomerados foram caracterizados utilizando dois indicadores $c_{S B} \mathrm{e}$ $\langle w\rangle$. O primeiro de sistemas cool-core (CC) e o outro de assimetrias morfológicas da emissão em raios-X. 
Catalogo de Aglomerados - Distribuições

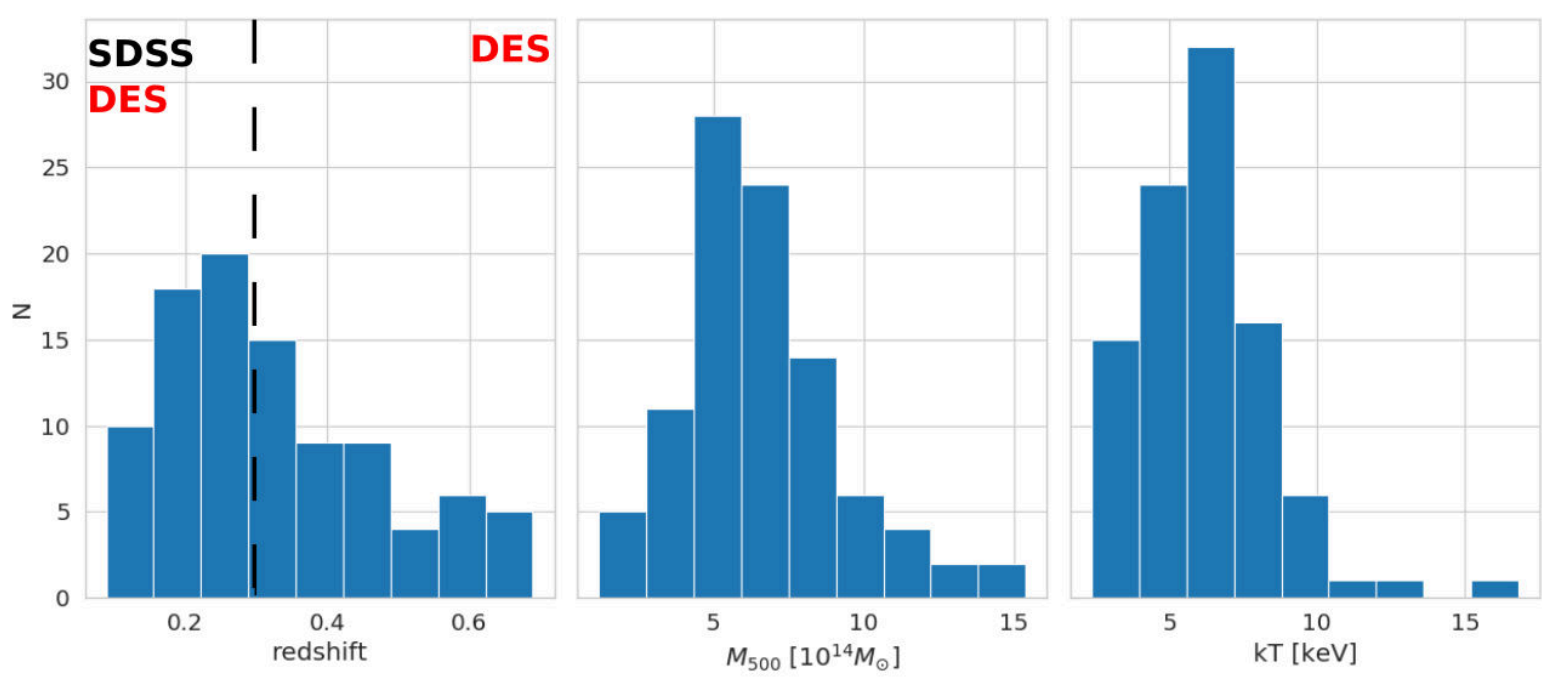

Figura 2.5: Distribuições de redshift, massa e temperatura. Nossa amostra conta com um total de 96 aglomerados, sendo composta pelos dados fotométricos dos SDSS e DES para $z<0.35$ (linha tracejada) e apenas dados do DES acima desse intervalo de redshift. 
Capítulo 3

\section{Análises em raios-X: Chandra}

O objetivo dessa seção é descrever os passos técnicos desenvolvidos até a obtenção das propriedades físicas dos aglomerados de galáxias usados neste trabalho, sendo tais propriedades: massa total, massa do gás, temperatura e os parâmetros de estado dinâmico.

As observações do Chandra têm suas informações sintetizadas em arquivo de eventos (em inglês event files). A cada fóton detectado é registrado um evento que tem informação do momento da detecção, posição no detector, nível do pulso gerado e a graduação ou padrão do evento. O nível do pulso gerado é convertido depois em energia do fóton incidente, e a graduação é uma nota ligada a probabilidade do evento ser de fato um fóton e não uma partícula energética. Essas informações são armazenadas em um arquivo com extensão fits, seguindo uma estrutura hierárquica de dados, da qual é possível gerar imagens, espectros e curvas de luz (variabilidade da emissão no tempo). A fim de facilitar a extração desses dados, existe o software CIAO ${ }^{1}$ (Fruscione et al., 2006) que possui uma vasta gama de funções desenvolvidas especialmente para lidar com produtos do telescópio espacial Chandra.

Neste trabalho utilizamos o sofwtare Chandra Source Catalog para acessar as propriedades das fontes pontuais em raios-X das observações. Para análise de fontes extensas, especificamente para aglomerados de galáxias, durante o mestrado foi desenvolvido o Xpipe, um algoritmo em Python que automatiza os processos de redução e análise apresentados nesta seção - disponível no githul². Para fins didáticos, as seções a seguir foram divididas de acordo com os 4 módulos do código assim como na estrutura do código. O Diagrama 3.1 ilustra essa estrutura. Durante essa análise foi usada a versão 4.7.9 CALDB dos ar-

\footnotetext{
1 http://cxc.harvard.edu/ciao4.10/

2 Xpipe: https://github.com/esteves jh/Xpipe
} 


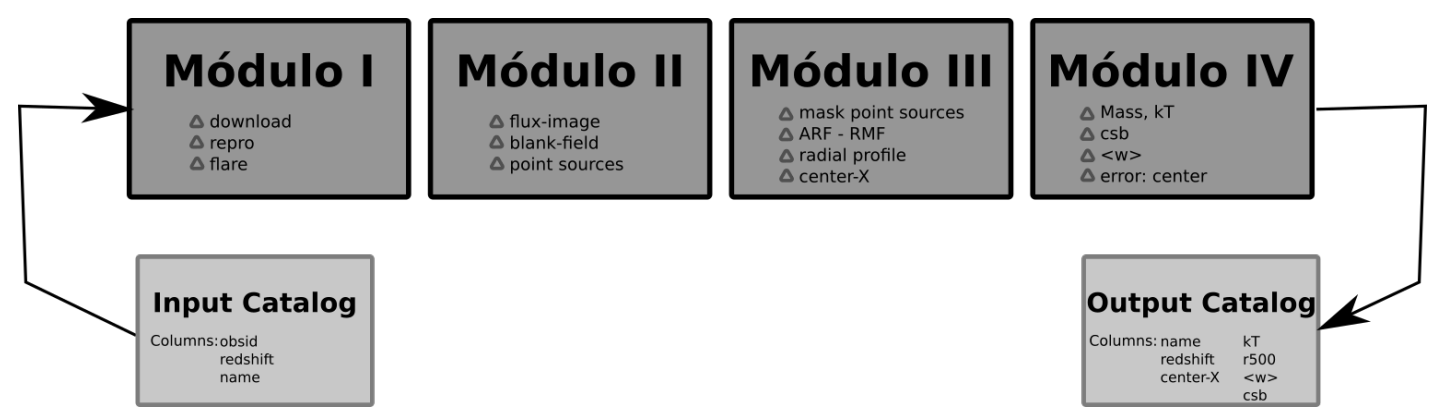

Figura 3.1: O diagrama ilustra o fluxo do algorítimo Xpipe que é dividido em quatro módulos. A entrada do algorítimo é uma tabela com informações do número de identificação do Chandra, OBSID, o redshift e nome do objeto. A saída é um catálogo com as informações de entrada mais as seguintes propriedades físicas estimadas: posição do centro, erro da posição do centro, temperatura, massa, massa do gás e indicadores do estado dinâmico, i.é. $\langle w\rangle$ e $c_{S B}$.

quivos de calibração. Também, em cada processo realizado foi explicitada a função CIAO utilizada.

\subsection{Módulo 1: redução de dados}

Este módulo realiza o pré-processamento que basicamente é a recalibração dos arquivos de eventos e a limpeza das observações por contaminações de flares que são surtos de partículas de fundo.

As observações são baixadas pela tarefa download_chandra_obsid. Depois, os arquivos são reprocessados com a tarefa chadra_repro. Essa etapa é importante para recalibrar os arquivos de eventos de acordo com a versão CALDB utilizada.

Uma parte importante da redução dos dados é a limpeza por contaminações de flares. Aglomerados de galáxias não têm variação de brilho em raios-X, portanto, se existe variações na curva de luz isto é um indicador de que houve algum surto de atividade solar ou um fluxo de partículas energéticas atingiu o detector. Para esse fim, filtra-se a observação deixando apenas os intervalos de observação onde a taxa de contagens não difere muito da média. Esses intervalos são definidos usando a função lc_clean que basicamente exclui todos períodos acima de $3 \sigma$ e/ou que estejam acima de $20 \%$ da média de contagens. É importante ressaltar que em casos extremos essa definição não é efetiva e pode levar a um erro considerável na estimativa da temperatura.

Na Figura 3.2 temos o exemplo do gráfico de saída da função lc_clean para dois aglomerados. No primeiro caso, notamos três períodos de flares. Enquanto no segundo caso, 

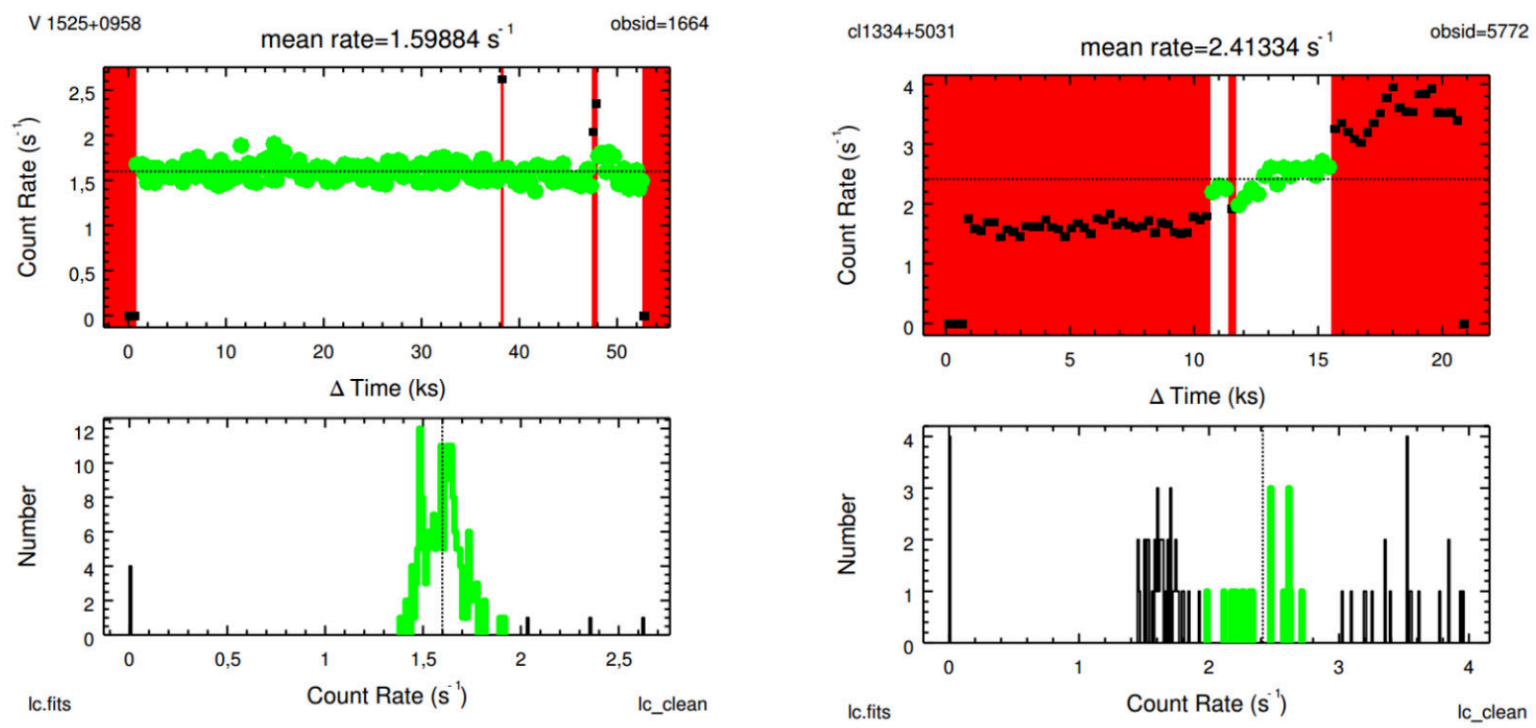

Figura 3.2: Limpeza por contaminações de flares. O gráfico da esquerda se refere ao aglomerado RX $\mathrm{J} 1525+0958, \mathrm{OBSID}=1664$ e da direita ao aglomerado CLJ1334+5031, OBSID=5772. Parte superior: curva de luz em contagens por segundo em função do tempo de exposição para duas observações com o detector ACIS-I. Parte inferior: distribuição de taxa de contagens. Os pontos verdes remetem aos períodos que estão dentro de $3 \sigma$ em torno da média, enquanto, as regiões vermelhas indicam os períodos de observações descartados.

temos uma situação excepcional, o método automático de limpeza dessa contaminação não estima corretamente o período de observação do aglomerado, pois, depois de $10 \mathrm{ks}$ de observação o detector é atingindo por um surto de partículas que cresce interruptamente até o fim da observação. Em particular, a temperatura estimada desse aglomerado, CLJ1334+5031, é $16 \mathrm{keV}$ considerando o método automático de limpeza e de 6 keV após a limpeza realizada manualmente (Maughan et al., 2012).

\subsection{Módulo 2: imageamento}

Nesta seção descrevemos como criamos a imagem de fluxo, a de background e encontramos as fontes pontuais.

No escopo deste trabalho as imagens em raios-X são importantes para o ajuste do perfil radial de fontes extensas. A fim de evidenciar a emissão da fonte, as imagem foram criadas no intervalo de energia entre $0.7-2.0 \mathrm{keV}$. Do ponto de vista técnico, antes da criação da imagem em raios-X existem uma série de passos. Após a limpeza de flares do arquivo de eventos se realiza a escolha campo de observação (i.e., o instrumento e os chips, conforme descritos na Figura 2.2), o tamanho do bin (tamanho do pixel) e do intervalo de energia. 
Como a observação é feita no espaço, é preciso se levar em conta o movimento orbital do telescópio, pois o detector capta a mesma região no céu em diferentes posições de sua órbita. Consequentemente, a área efetiva e o tempo de observação ao longo da imagem não são uniformes, o que implica na existência de pequenas variações entre as imagens. Além disso, a área efetiva do detector também depende da energia da partícula incidente.

O mapa de exposição é uma imagem que leva em conta essas variações - análogo ao flat-field comumente utilizado em imagens ópticas - e possui unidades $\left[\mathrm{s} \mathrm{cm}^{2}\right]$. Para sua criação é necessário o valor do pico de energia da observação, que é por volta de $1.7 \mathrm{keV}$. Além de levar em conta a variação da área efetiva do detector, o mapa de exposição também tem informações do detector (p.ex., pixeis ou colunas de pixeis que não funcionam). Por fim, a imagem corrigida é a divisão da imagem criada numa banda de energia pelo mapa de exposição, assim, temos uma imagem calibrada em fluxo, com unidades de fótons $\mathrm{s}^{-1} \mathrm{~cm}^{-2}$.

Na prática, a tarefa fluximage resume todos os passos descritos acima para observações únicas. Por outro lado, para observações múltiplas temos um mosaico de imagens com a função merge_obs. Como no caso do mosaico existem várias observações, é necessário definir um ponto em comum de projeção das imagens. Geralmente, se escolhe a coordenada do aglomerado. Na Figura 3.3 temos um exemplo de um mosaico de imagens corrigidas por mapa de exposição.

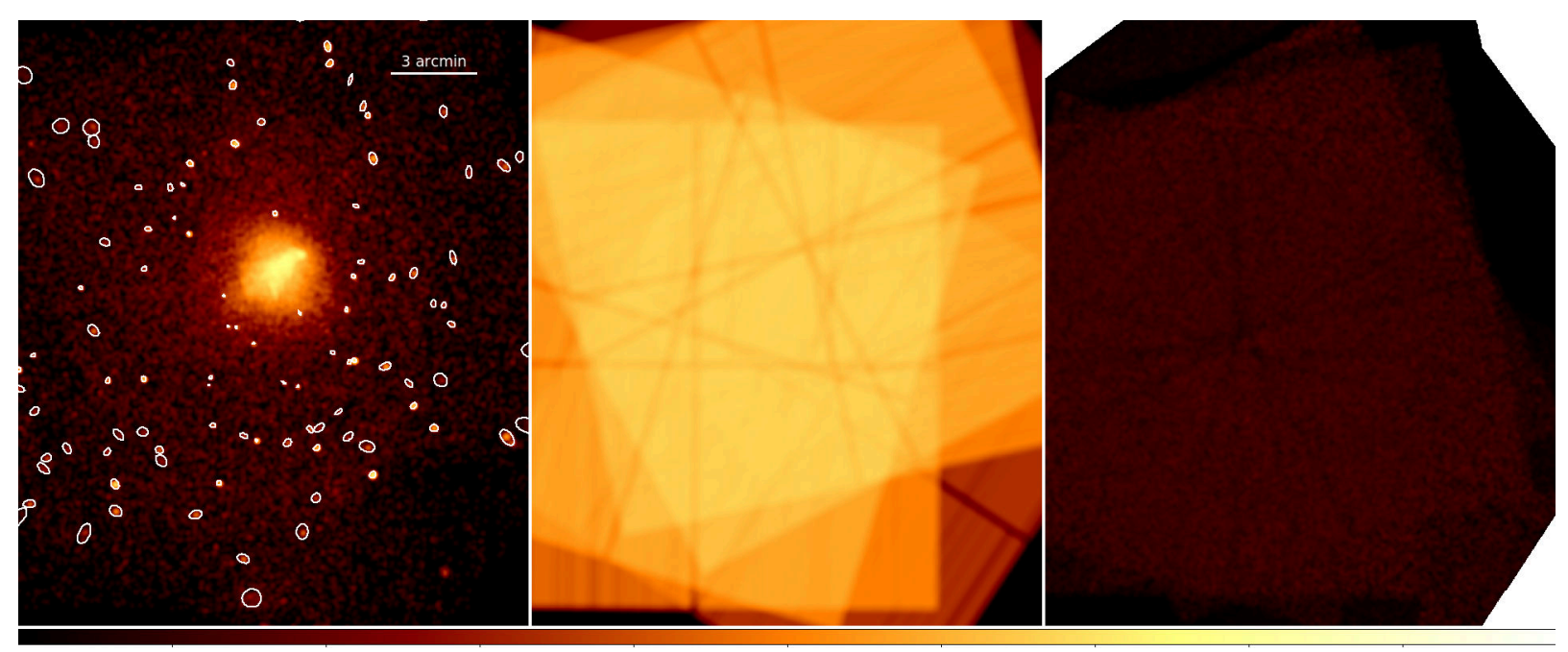

Figura 3.3: Múltiplas observações em raios-X do Aglomerado da Bala (mais conhecido como Bullet Cluster). Esquerda: imagem suavizada por um filtro Gaussiano e corrigida pelo mapa de exposição, em branco são elipses em torno das fontes pontuais. Centro: sobreposição dos mapas de exposições. Direita: sobreposições das imagens de blank-sky - usada para a subtração da contaminação de emissão de fundo.

O background é uma parte importante da análise de imagens e também do ajuste 
espectral que pode ser feito de algumas maneiras distintas. Nesse trabalho usamos blanksky que são observações profundas de regiões aleatórias do céu onde não se tem nenhuma emissão extensa detectável em raios-X. Esses dados são calibrados com os mesmos arquivos de calibração do arquivo de eventos e suas imagens são projetadas na mesma direção da imagem original. Além disso, também é feita na imagem uma normalização da taxa de contagens na parte mais energética do espectro, na banda 9.5-12 keV. Esse intervalo é interessante para este fim porque não há praticamente emissão do aglomerado, salvo para aglomerados com uma altíssima temperatura. Esses tipos de observações são obtidos com as funções blanksky e blanksky_image. Na Figura 3.3, em particular, na imagem da direita temos um exemplo de blank-sky.

As fontes pontuais são detectadas em imagens corrigidas pelo mapa de exposição, usando a função wavdetect. No nosso trabalho usamos um filtro gaussiano com escalas 2, 2.828, 4, 5.657, 8, 11.314 e 16 pixeis. Após a identificação, verificamos as detecções em imagens suavizadas, similar a imagem da esquerda na Figura 3.3. Posteriormente, excluímos as fontes pontuais que foram verificadas.

\subsection{Módulo 3: pré-análise}

Nesta seção descrevemos os passos que precedem a análise, ou seja, o ajuste espectral e o ajuste de brilho superficial.

\subsubsection{Centro em Raios- $X$}

O centro em raios-X é determinado como o centroide de luminosidade de emissão em raios-X. O procedimento é feito iterativamente em um círculo de raio igual a $500 \mathrm{kpc}$ primeiramente, no fim, e refeito em uma abertura de raio $R_{500}$. O centroide é encontrado em uma imagem suavizadas por um filtro gaussiano de $5 \sigma$, se repete o procedimento até atingir uma convergência menor que um pixel. Lembrando que todas fontes pontuais são mascaradas. Outra medida de centro é o pico em raios-X que é utilizado no caculo do parâmetro desvio do centro, a sua posição é computada como o pixel mais brilhante da imagem suavizada em uma abertura de $500 \mathrm{kpc}$.

Em geral, a acurácia das medidas do centro é proporcional ao tamanho da PSF do instrumento, cerca de $0.5 \%$. No entanto, para imagens com pouca contagem, o ruído 
Poissoniano pode ser um efeito dominante. Afim de quantificar essa sistemática, calculamos a incerteza na posição por um método de Monte Carlo das imagens em raios-X. Maughan et al. (2012) utilizou esse técnica para estimar erro das medidas de desvio do centro $\langle w\rangle$. Esse método consiste em gerar um ruído aleatório de distribuição Poissoniana em cada pixel da imagem, e realizar a medida do centro em raios-X. Se repete o procedimento 100 vezes, por fim, a incerteza é definida como o desvio padrão da distribuição de medidas do centro. Na Figura 3.4 mostramos uma imagem sem o ruído, uma com ruído e depois a subtração das imagens. Podemos perceber que as estruturas mais fracas mudam a forma com a adição do ruído, mais o pico é pouco afetado.

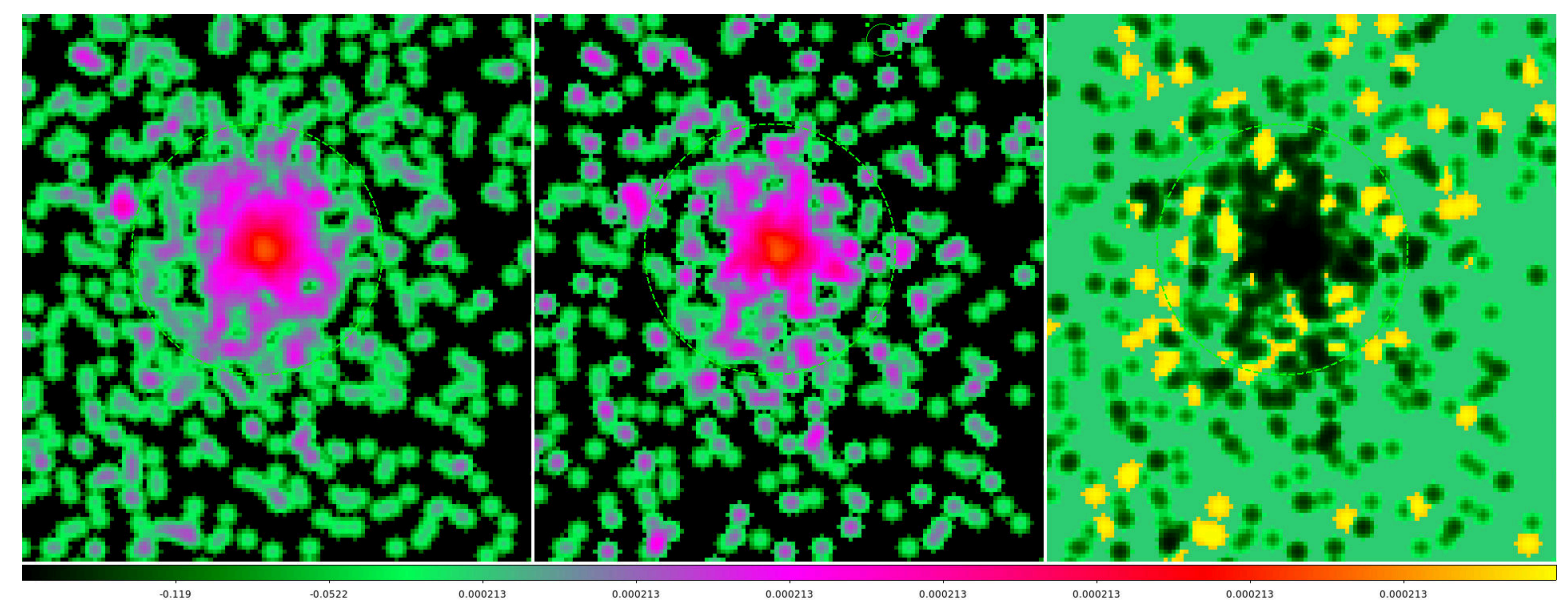

Figura 3.4: Determinação do centro para o aglomerado do redMaPPer de MEM_MATCH_ID= 1997 em $z=0.3$. O circulo de linhas tracejadas verde tem 1 minuto de arco de abertura e centro na posição do centroide de luminosidade de emissão em raios-X. Esquerda: a imagem suavizada por um filtro Gaussiano corrigida por mapa de exposição. Centro: a imagem da direita com adição de um ruído Poissoniano, posteriormente suavizada num filtro Gaussiano. Direita: é a imagem residual. Nota-se que as estruturas mais fracas mudam a forma com a adição do ruído, mas o pico é pouco afetado.

\subsubsection{Anéis}

O perfil radial de brilho superficial é medido em anéis concêntricos centrado no pico em raios-X, porém, também pode ser feito a partir do centroide de luminosidade. O ajuste deste perfil pode depender da forma como se define a espessura dos anéis.

Em nosso trabalho, criamos perfis com um número fixo de pontos, algo por volta de 40. Para tal, os anéis criados tem o mesmo número de contagens. Os anéis concêntricos se estendem até a borda do detector. Em particular, encontramos o raio máximo para cada observação. Onde este raio é a menor distância do centro do aglomerado até a borda. 
Por fim, o perfil é extraído da imagem corrigida por mapa de exposição e com as fontes pontuais mascaradas. A função dmextract é usada para criar esses perfis.

\subsubsection{Arquivos Auxiliares: ARF e RMF}

A sensibilidade do detector é dada em termos de área efetiva, medida em centímetros quadrado. O arquivo, ARF (do inglês, "Ancillary Response File") contém a informação relativa à eficiência quântica, o filtro e a área do detector combinadas no termo genérico de área efetiva. A Figura 3.5 ilustra a dependência da área efetiva em relação à energia, assim como, a diferença para dois detectores. É importante lembrar que a dependência também é espacial, pois, quanto mais perto da borda uma fonte é observada - i.e., mais distante do eixo do telescópio - menor é a sensibilidade do detector. Para levar em conta esse efeito, a função é calculada para cada ponto do detector.

O caminho de um fóton de raios-X até a sua detecção como um evento é permeado por uma série de efeitos devidos à PSF, eletrônica, espelhos do telescópio e detector. Considerando um dado número de fótons de energia $E$ que foram emitidos por uma aglomerado, a detecção dessas partículas é um outro número de contagens para uma dada unidade de área e uma dada unidade de tempo em um canal de energia $i$. Essa relação entre a energia do fóton originalmente emitido e o canal de energia detectado se refere pela chamada matriz de redistribuição.
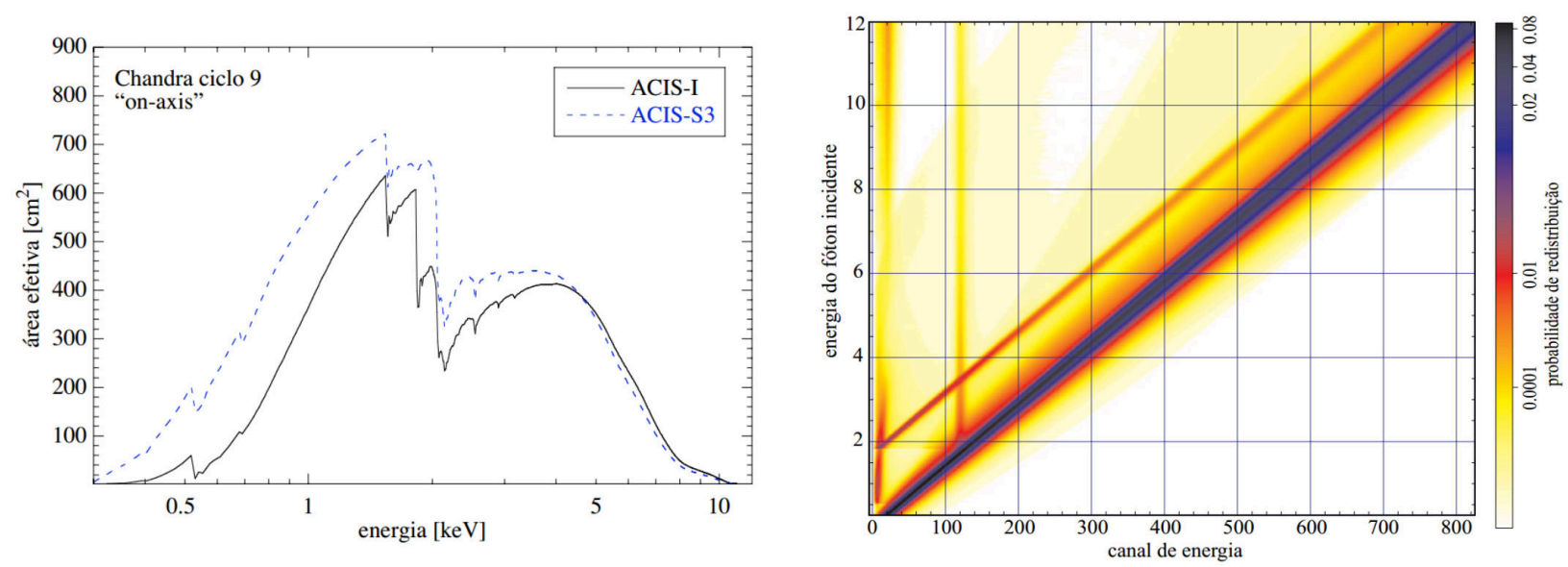

Figura 3.5: Esquerda: área efetiva dos detectores ACIS-I e ACIS-S3 (chip back illuminated mais próximo do eixo do telescópio e com melhor foco) em função da energia para uma fonte no eixo do telescópio. Direita: exemplo de uma matriz de redistribuição, aqui a RMF do detector ACIS-I, para uma observação de uma fonte no eixo do telescópio. A abscissa é o canal de energia e a ordenada é a energia do fóton. A relação entre os dois é uma probabilidade representada pela cor da figura. Fonte: Lima Neto (2009). 
Por convenção, a matriz é armazenada em um arquivo fits e a RMF (do inglês, Redistribution Matrix File) é interpretada como a probabilidade do fóton de energia $E$ ser detectado no canal $i$. Em um detector perfeito, a matriz de redistribuição deveria ser uma relação biunívoca entre energia e canal, porém, detectores como ACIS são casos intermediários, a sua relação é quase diagonal, e possui picos de probabilidade para canais de baixa energia, onde mesmo fótons energéticos são falsamente detectados. A Figura 3.5 ilustra um exemplo dessa relação.

Multiplicando um espectro teórico pela ARF obtemos o espectro observado pelo telescópio, sem levar em conta a resolução espectral finita. Para leva-la em conta, utilizamos a RMF. Com estas duas funções da energia, se produz o espectro ponderado pela área efetiva do telescópio e pela eficiência do detector. Sendo assim, a função specextract produz os arquivos auxiliares ARF e RMF juntamente com o espectro.

\subsection{Módulo 4: análise}

Nesta seção descrevemos os passos da análise. Mostramos primeiramente, como é feito o ajuste espectral e o ajuste do brilho superficial. Por fim, descrevemos determinação as propriedades do aglomerado como temperatura, massa, massa do gás e parâmetros do estado dinâmico.

\subsubsection{Ajuste Espectral}

Foi utilizado o pacote Sherpa que é o algoritmo de ajuste do CIAO (Freeman et al. 2001). Este pacote utiliza as bibliotecas do xsped ${ }^{3}$ para o ajuste espectral. O principal método de optimização utilizado é o Levenberg-Marquardt (Moré, 1978).

O ajuste espectral foi realizado por um modelo MEKAL no intervalo de energia 0.3$8.0 \mathrm{keV}$. A temperatura e a normalização do modelo são livres pra variar, enquanto a abundância de metais é fixa em $0.3 Z_{\odot}$ e a coluna de hidrogênio fixa no valor galáctico inferido pelas observações da linha $21 \mathrm{~cm}$ de hidrogênio (Dickey e Lockman, 1990). O background é subtraído utilizando o blank-sky. Embora a metalicidade poderia ser ajustada em observações com boa qualidade, decidimos fixá-la devido ao grande números de casos de observações pobres em contagens.

\footnotetext{
${ }^{3}$ home page.https://heasarc.gsfc.nasa.gov/xanadu/xspec/
} 
O fator de normalização do espectro é dado pela seguinte expressão:

$$
n o r m=\frac{10^{-14}}{4 \pi\left[D_{A}(1+z)\right]^{2}} \int n_{e} n_{p} d V,
$$

onde $n_{e}$ e $n_{p}$ são as densidades numéricas de elétrons e prótons, respectivamente. Sendo $D_{A}$ a distância angular. O fator norm é utilizado para transformar as unidades de contagens para fluxo.

\subsubsection{Ajuste do Perfil de Brilho Superficial}

O perfil de brilho superficial é ajustado por um modelo $\beta$-modificado (Maughan et al. 2008):

$$
n_{p} n_{e}=\left(n_{0}\right)^{2} \frac{\left(r / r_{c}\right)^{-\alpha}}{\left(1+r^{2} / r_{c}^{2}\right)^{3 \beta-\alpha / 2}} \times\left(1+\left(r^{\gamma} / r_{s}^{\gamma}\right)\right)^{-\epsilon / \gamma}
$$

O modelo tem ao todo seis parâmetros, dois radiais, $r_{s}, r_{c}$, e quatro parâmetros expoentes positivos, $\alpha, \beta, \gamma, \epsilon$. Todos são livres para variar, com exceção de $\gamma$ que é fixo em 3 como sugerido por Vikhlinin et al. (2006). Esse modelo consiste em um modelo beta mais a adição de uma lei de potência no centro e de um fator que regula a queda do brilho em regiões mais externas. Essas alterações são interessantes pois, propiciam maior flexibilidade ao ajuste de sistemas cool-core.

A equação 3.2 é projetada ao longo da linha de visada e posteriormente ajustada ao brilho superficial. A projeção ao longo da linha de visada é feita pela clássica transformada de Abel. Assumindo simetria esférica o brilho superficial é dado por:

$$
S_{X}(R) \propto \int_{R}^{\infty} n_{p} n_{e}(r) \times \frac{2 r^{2}}{\sqrt{r^{2}-R^{2}}} d r
$$

No fim, apenas ajustamos a forma do perfil. O fator de normalização $n_{0}$ é calculado depois do ajuste. De fato, com os valores do ajuste do modelo se estima a normalização, norm, segundo a equação 3.1. E tomando a razão com o valor de normalização encontrado no ajuste espectral se tem $n_{0}^{2}$. Por fim, temos o perfil em unidades de fluxo.

\subsubsection{Determinação de $M_{500}$}

A massa do aglomerado em raios-X pode ser estimada de algumas maneiras. Uma estimativa simples é utilizar a relação massa-temperatura, $k T-M$, porém temos que a relação não é acurada, possui um espalhamento por volta de 30\% (Arnaud et al., 2005). 
Em nosso trabalho utilizamos a relação de escala $Y_{X}-M$ para estimar a massa do aglomerado Vikhlinin et al. (2009), onde $Y_{X}=M_{g a s, 500} \times k T_{\text {spec }}$. A escolha é motivada pelo fato de a relação possuir uma baixa espalhamento (cerca de 7\%) e ser insesível a sistemas em colisões Kravtsov et al. (2006) - o que é uma vantagem para uma amostra heterogênea como a utilizada aqui.

A temperatura é estimada conforme descrito na seção anterior, considerando a banda entre $0.3-8 \mathrm{keV}$ e em um anel de abertura $(0.15-1) \times R_{500}$.

A massa do gás é estimada com o melhor ajuste do perfil $\beta$-modificado, e se assumindo uma relação $n_{e} / n_{p}=1.1195$ para uma metalicidade típica de $0.3 Z_{\odot}$ (Anders \& Grevesse 1989). Com simetria esférica, temos:

$$
M_{g a s, 500}(<R)=\mu_{e} m_{p} \int n_{e}\left(r^{\prime}\right) d V=1.151 m_{p} \int_{0}^{R} 4 \pi n_{e}\left(r^{\prime}\right) r^{\prime 2} d r^{\prime},
$$

lembrando que $\mu_{e}$ é o peso molecular médio.

Sendo assim, podemos calcular a massa pela seguinte relação:

$$
M_{500, Y_{X}}=E^{-2 / 5}(z) A_{Y M}\left(\frac{Y_{X}}{3 \times 10^{14} M_{\odot} \mathrm{keV}}\right)^{B_{Y M}},
$$

onde $A_{Y M}=5.77 \times 10^{14} h^{1 / 2} M_{\odot}$ and $B_{Y M}=0.57$ (Maughan et al. 2012). Sendo $E(z)$ a razão da constante de Hubble no redshift $z$ divida pelo seu valor presente.

Finalmente, $R_{500}$ é computado:

$$
M_{500, Y_{X}} \equiv 500 \rho_{c}(4 \pi / 3) R_{500}^{3}
$$

O procedimento é repetido iterativamente até se atingir uma diferença fracionaria menor de $1 \%$ no raio.

\subsubsection{Desvio do centro: $\langle w\rangle$}

O desvio do centro é definido como o desvio padrão da diferença do pico em raios-X e o centroide, sendo este último calculado em uma serie de aberturas circulares centradas no pico em raios-X e um raio diminuindo em $5 \%$ de $R_{500}$ até $0.05 \times R_{500}$.

$$
\langle w\rangle=\frac{1}{R_{500}} \sqrt{\frac{1}{N-1} \sum_{i}\left(\Delta_{i}-\bar{\Delta}\right)^{2}}
$$


Seguindo o método de Maughan et al. (2012), estimamos $\langle w\rangle$ segunda a equação 3.6. A medida foi em 100 repetições adicionando uma distribuição aleatória de Poisson de pixeis em cada imagem em raios-X.

\subsubsection{Concentração: $c_{S B}$}

O parâmetro concentração indica o quanto a emissão em raios-X é concentrada no centro. Foi usado recentemente por Andrade-Santos et al. (2017) por fim de estimar a fração de aglomerados de núcleo frio. Sua medida é simples, a razão de perfil de brilho superficial conforme a equação:

$$
c_{S B}=\frac{\Sigma\left(<0.15 R_{500}\right)}{\Sigma\left(<R_{500}\right)}
$$

\subsection{Xpipe: pipeline em raios- $X$}

Nesta seção descrevemos o código Xpipe desenvolvido para automatizar os procedimentos de análise de fontes extensas em raios-X até a obtenção das propriedades físicas.

Para facilitar o uso, criamos um algorítimo em módulos e com arquivo de configuração. Além disso, tem um modo paralelizado para o ajuste de uma grande quantidade de objetos.

Os módulos do código são divididos em quatro conforme descrito no diagrama 3.1 . Descrevemos os passos realizados em cada módulo nas seções acima desse capítulo.

O arquivo de configuração Xpipe_config. ini facilita o uso do código. Nesse documento de texto, o usuário tem a opção de inserir os dados de entrada e quais módulos do código ira processar. No Apêndice 7.1 temos um exemplo desse arquivo. O catálogo de entrada é inserido na seção Files, na variável catInputFile e pode ter os formatos csv ou fits. Os diretórios de saída estão na seção Paths. Foi divido em dois, outputPath e dataDownPath. O primeiro é o diretório que se armazenam os arquivos criados nos módulos do código. O segundo é o diretório onde os dados são baixados da base de dados do Chandra. Os subdiretórios dessas pastas tem nomenclaturas diferentes. Em outputPath para cada aglomerado do catálogo de entrada é criado uma pasta como o nome da coluna ID - nome do aglomerado. Em dataDownPath temos que o nome de cada subdiretório é o número de identificação da observação, obsid. 


\subsection{Fontes Pontuais: AGNS}

Nesta seção descrevemos o modelo de emissão em raios-X de AGNs e também a relação do hardness-ratio, cor em raios-X, com as galáxias obscurecidas.

Observações em raios-X fornecem um dos métodos mais confiáveis para identificar AGN. Isso é devido a emissão de raios $\mathrm{X}$ de outros processos astrofísicos ser tipicamente mais fraca em comparação a de AGNs (Hickox e Alexander, 2018). Os outros processos físicos emissores de raios-X, além das AGNs e o gás quente $T>10^{6} \mathrm{~K}$, são estrelas de nêutrons em processo de acreção e buracos negros de massa estelar, comumente chamados de binárias em raios-X (Fabbiano, 2006). Esses sistemas têm uma correlação da luminosidade em raios-X com a taxa de formação estelar (p. ex.: Lehmer et al., 2016). Geralmente, um corte de $L_{X}>10^{42} \mathrm{erg} / \mathrm{s}$ garante uma pureza da amostra de AGNs, pois apenas galáxias com fortíssimas taxas de formação estelar produzirão uma emissão em raios-X maior do que esse limite (Alexander et al., 2005).

Sendo assim, a identificação de AGNs em nosso trabalho é feita por fontes pontuais em raios-X com contrapartida óptica. Realizamos primeiramente a busca no Chandra Source Catalog de todas as fontes pontuais em raios-X da nossa amostra de aglomerado com um distância de até $4 \times R_{500}$. Aplicamos os seguintes cortes para garantir a precisão das fontes: detecções com erro na posição menor que 4"; e detecções que estejam abaixo de $10^{\prime}$ em relação ao eixo do telescópio. Depois, cruzamos esse catálogo com o catálogo de galáxias no óptico. Além disso, a fim de separar as galáxias do aglomerado dos objetos de fundo, utilizamos um método probabilístico para identificar as galáxias membros. Identificamos como AGNs apenas objetos que tem um probabilidade de redshift maior que 0.1. Esse método probabilístico será apresentado no próximo capitulo.

\subsubsection{AGNs Obscurecidas}

A observação em raios-X também é um método muito confiável para identificar AGNs obscurecidas, isto é, galáxias hospedeiras com alta absorção na linha de visada. A absorção é causada por poeira nos comprimentos de onda do ultravioleta ao infravermelho e pelo gás em raios-X. Esse obscurecimento pode vir da regiões próximas ao disco de acresção ou outras regiões da galáxia hospedeira, por exemplo, regiões de formação estelar obscurecidas por poeira. Pode ser mais significativa para galáxias em processo de fusão ou em galáxias 
edge-on (Hickox e Alexander, 2018).

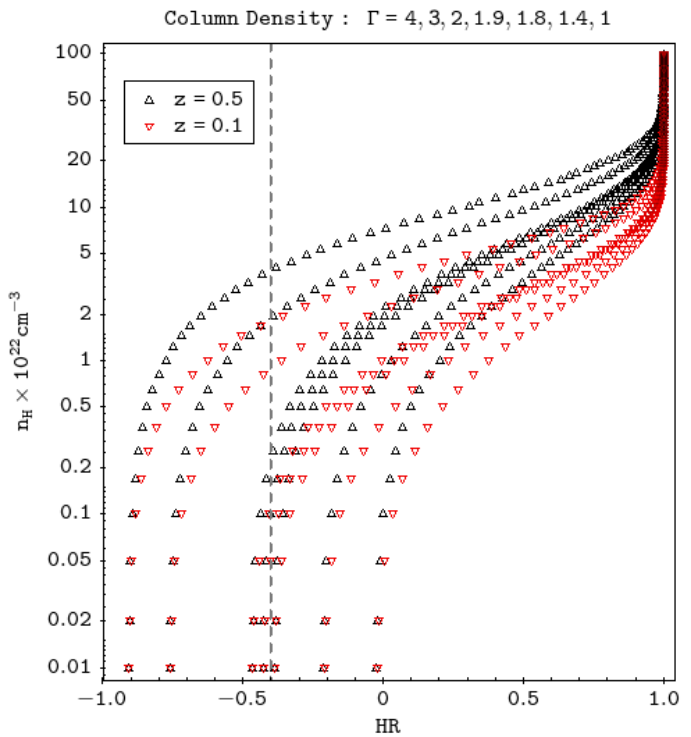

Figura 3.6: Relação coluna de hidrogênio $\left(n_{H}\right)$-hardness-ratio (HR). Assumimos um modelo de lei de potência mais um modelo de absorção, com ajuda do xspec simulamos observações de HR para o Chandra. Variamos a inclinação do espectro, $\Gamma$, entre 1 e 4 estes valores correspondem as curvas da direita pra esquerda. Quanto maior $\Gamma$ menor HR. As curvas são para uma galáxia hospedeira em $z=0.1 \mathrm{e}$ $z=0.5$. Não notamos grandes diferenças com o redshift.

A "cor" em raios-X, hadness-ratio(HR), é um bom método para encontrar AGNs obscurecidas em observações com pouca contagem. Existe uma relação bem conhecida entre a coluna de hidrogênio, $n_{H}$ e o HR. De fato, geramos curvas dessa relação $n_{H}$-HR assumindo modelos de leis de potência mais um modelo de absorção, típicos para a modelagem da emissão de AGNs em raios-X. Os valores de HR foram obtidos usando o xspec para ARF e RMF padrões do Chandra. A Figura 3.6 mostra essa relação para dois casos, uma galáxia hospedeira em $z=0.1$ e outra em $z=0.5$, em ambos o casos variamos a inclinação do espectro entre 1 e 4 . É evidente que para as inclinações típicas de AGN, $1<\Gamma<2$, valores de HR maior que 0 assumem $n_{H}>10^{22} \mathrm{~cm}^{-3}$. Um intervalo padrão adotado é $H S>-0.25$ para classificar objetos obscurecidos (Hickox e Alexander, 2018).

\subsubsection{Luminosidade em raios- $X$}

Calculamos a luminosidade das fontes pontuais em raios- $\mathrm{X}$ no referencial de repouso na banda broad, 0.5-7.0 keV. Assumimos um modelo de lei de potência e inclinação do espectro, $\Gamma=2$.

$$
L_{0.5-7 k e V}=4 \pi D_{L}^{2} F_{0.5-7 k e V}(1+z)^{\Gamma-2}
$$




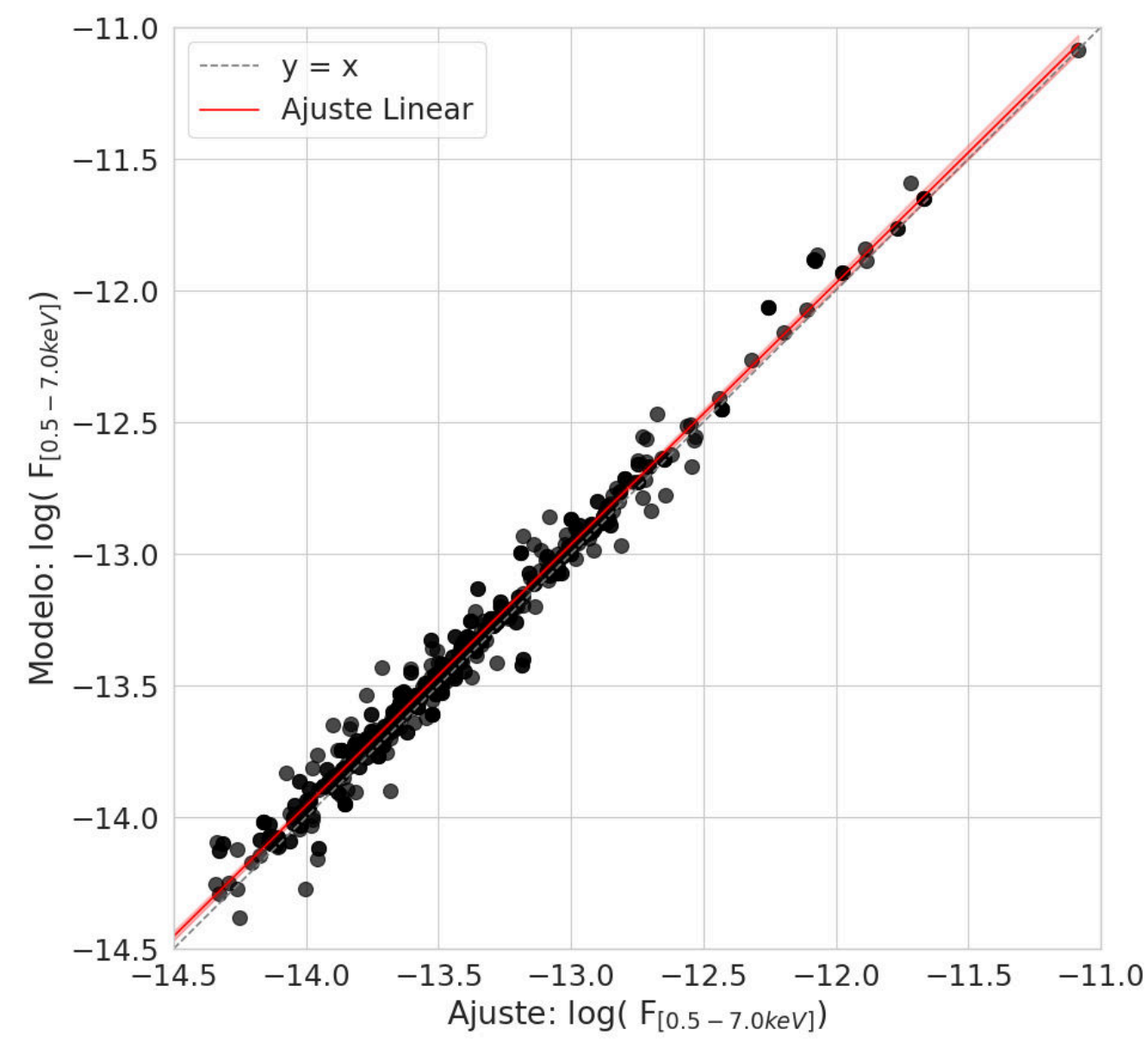

Figura 3.7: Comparação de duas estimativas de fluxo assumindo uma lei de potência mais um modelo de absorção para uma amostra seleta de fontes pontuais em raios-X. No eixo $\mathrm{X}$ temos o fluxo estimado pelo ajuste espectral ajustando a inclinação do espectro e a densidade de coluna de hidrogênio da galáxia hospedeira, $n_{H}$. No eixo Y temos o fluxo estimado a inclinação do espectro igual a 2 e a absorção da galáxias hospedeira não é considerada. A linha traceja em preto indica uma relação um para um e a linha vermelha é o melhor ajuste linear dos pontos.

Onde $D_{L}$ é a distância de luminosidade. O redshift usado foi o do aglomerado.

Utilizamos os fluxos, $F_{0.5-7 \mathrm{keV}}$, estimados pelo Chandra Source Catalog(CSC) que assume um modelo com $\Gamma$ fixo em 2 e uma absorção intrínseca da via-láctea, sendo assim desconsideram a absorção da galáxia hospedeira. Apesar de trabalhos dessa natureza utilizarem $\Gamma=1.8$ (Alexander et al., 2005), decidimos manter o padrão do CSC por simplicidade. Fizemos um teste, para uma amostra seleta de fontes com contagens suficientes para um ajuste espectral, comparamos os fluxos estimados com parâmetros fixos e com valores ajustados, ambas estimativas providas pelo CSC. Não encontramos mudanças significativas que possam comprometer o nosso trabalho, veja Figura 3.7. 
Capítulo 4

\section{Análise no Óptico}

\subsection{Seleção de Galáxias Membro}

Utilizamos um método probabilístico para selecionar as galáxias membro de aglomerados de galáxias. Essa metodologia foi desenvolvida e usada nos trabalhos Pereira et al. (2018) e Palmese et al. (2019).

Basicamente o método consiste em atribuir duas probabilidades para cada galáxia na região do aglomerado que são relativas ao redshift e a posição radial. Então, a probabilidade de uma galáxia ser membro do aglomerado é definido como o produto dessas probabilidades,

$$
P_{m}=P(z) \times P(R) .
$$

Nas próximas seções definimos e comentamos brevemente sobre cada probabilidade.

\subsubsection{Probabilidade de Redshift}

Afim de distinguir galáxias de fundo ou pertencentes ao aglomerado, a probabilidade de redshift é calculada integrando uma PDF (do inglês, Probabilistic Distribution Function) Gaussiana em um intervalo ao redor do redshift do aglomerado, $z_{0}$. O valor médio da PDF é o redshift fotométrico da galáxia $z$ e o desvio padrão é a incerteza do redshift fotométrico $\delta z$, ou seja,

$$
P(z)=\int_{z_{\min }}^{z_{\max }} \frac{1}{\sqrt{2 \pi}\left(\delta z^{\prime}\right)} e^{-f\left(z^{\prime}\right)} d z^{\prime}
$$

onde a função $f\left(z^{\prime}\right)$ é dada por :

$$
f\left(z^{\prime}\right)=\frac{\left(z^{\prime}-z_{0}\right)^{2}}{2\left(\delta z^{\prime}\right)^{2}}
$$

Para levar em conta as mudanças da incerteza no redshift fotométrico para diferentes intervalos de redshift, o tamanho do intervalo de integração muda baseado na mediana 
$\langle\delta z\rangle$ das galáxias em nossa amostra conforme a equação abaixo:

$$
\begin{gathered}
z_{\min }=z_{0}-n(\langle\delta z\rangle) \times(1+z) \\
z_{\max }=z_{0}+n(\langle\delta z\rangle) \times(1+z) .
\end{gathered}
$$

Os valores típicos de $\langle\delta z\rangle$ são entre $0.09--0.11$. Em nosso trabalho tomamos o valor de $n=0.5$, e afim de evitar galáxias muito fora do intervalo de redshift do aglomerado, cortamos todas as galáxias fora do intervalo de redshift de $2\langle\delta z\rangle$. Essa probabilidade de redshift dá um peso maior as galáxias com redshift aproximadamente ao do aglomerado do que para galáxias com um redshift muito diferente. Além de tender a reduzir o peso para galaxias com grande incerteza no redshift fotométrico.

\subsubsection{Probabilidade Radial}

Podemos atribuir a cada galáxia uma probabilidade de pertencer ao aglomerado baseado na sua distância projetada $R$ e em relação a $R_{200}$. Para calcular a probabilidade radial, assumimos um perfil NFW projetado, no qual foi mostrado em Wright e Brainerd (2000) ser:

$$
\Sigma(R)= \begin{cases}\frac{2 \rho_{s} R_{s}}{r^{2}-1}\left[1-\frac{2}{\sqrt{r^{2}-1}} \arctan \sqrt{\frac{r-1}{r+1}}\right] & r>1 \\ \frac{2 \rho_{s} R_{s}}{3} & r=1 \\ \frac{2 \rho_{s} R_{s}}{r^{2}-1}\left[1-\frac{2}{\sqrt{1-r^{2}}} \operatorname{arctanh} \sqrt{\frac{1-r}{r+1}}\right] & r<1,\end{cases}
$$

onde $r=R / R_{s}$. Sendo $R_{s}$ é o raio característico projetado definido como

$$
R_{s}=\frac{R_{200}}{c}
$$

e $c$ é chamado de parâmetro de concentração. Afim de evitar a singularidade em $R=R_{s}$ definimos um raio do caroço $R_{\text {core }}=R_{s}$ e aproximamos a densidade central para uma constante.

Esta probabilidade é calculada em termos de $R_{200}$, porém, o ajuste em raios-X nos fornece $R_{500}$. Sendo assim, definimos o raio duzentos com o parâmetro de concentração fixo, $R_{200, c}$. Em nosso trabalho, assumimos $c=3$ e temos que $R_{200, c} \approx R_{500} / 0.65$.

A equação 4.5 na forma que está definida tem unidade de massa superficial, porém, estamos trabalhando com densidade numérica de galáxias em nossos cálculos. Portanto, é necessário um fator de conversão de unidades de massa por área para unidades de galáxias por unidade de área. Para tal, definimos a seguinte constante: 


$$
A \equiv\left(n_{t o t}-n_{b g}\right) / \int_{0}^{R_{200}} 2 \pi R^{\prime} \Sigma\left(R^{\prime}\right) d R^{\prime},
$$

onde $n_{t o t}$ é o número total de galáxias e $n_{b g}$ é o número de galáxias de fundo. A estimativa do número de galáxias de fundo é feito em um anel de $(4-6) \times R_{500}$.

Por fim, temos que a probabilidade radial é

$$
P(R)=\frac{A \Sigma(R)}{A \Sigma(R)+n_{b g}} .
$$

Em essência, isso nos dá a probabilidade de que uma galáxia pertença a um aglomerado em específico, dado a distância angular da galáxia no centro do aglomerado. Nota-se que assumimos uma simetria esférica.

\subsection{Distribuição de Cor}

Nesta seção descrevemos o ajuste da sequência vermelha e a definição de galáxias azuis e vermelhas aplicadas nesse trabalho.

\subsubsection{Limite de magnitude}

O limite de magnitude dependente do redshift é essencial para se comparar a evolução de um mesmo conjunto de galáxias. Sendo assim para definir a magnitude dependente do redshift, utilizamos o modelo de evolução de uma típica galáxia elíptica usando o algoritmo LePhare 1 .

Na Figura 4.1 temos a magnitude observada para um típica galáxia elíptica nos filtros $r, i$ e $z$ ao longo do redshift. Adotamos o corte como sendo $m_{\text {modelo }}+3$, sendo esse limite abaixo da magnitude limite do SDSS e do DES em todo o intervalo de redshift do nosso estudo.

\subsubsection{Sequência Vermelha}

A sequência vermelha (SV) é uma relação cor-magnitude encontrada em aglomerados. É composta majoritariamente por galáxias elípticas e S0. Além disso, a sua evolução ao longo do redshift pode ser predita em modelos de formação e evolução estelar. De forma

\footnotetext{
${ }^{1}$ home page:http://www.cfht.hawaii.edu/ arnouts/LEPHARE/lephare.html
} 


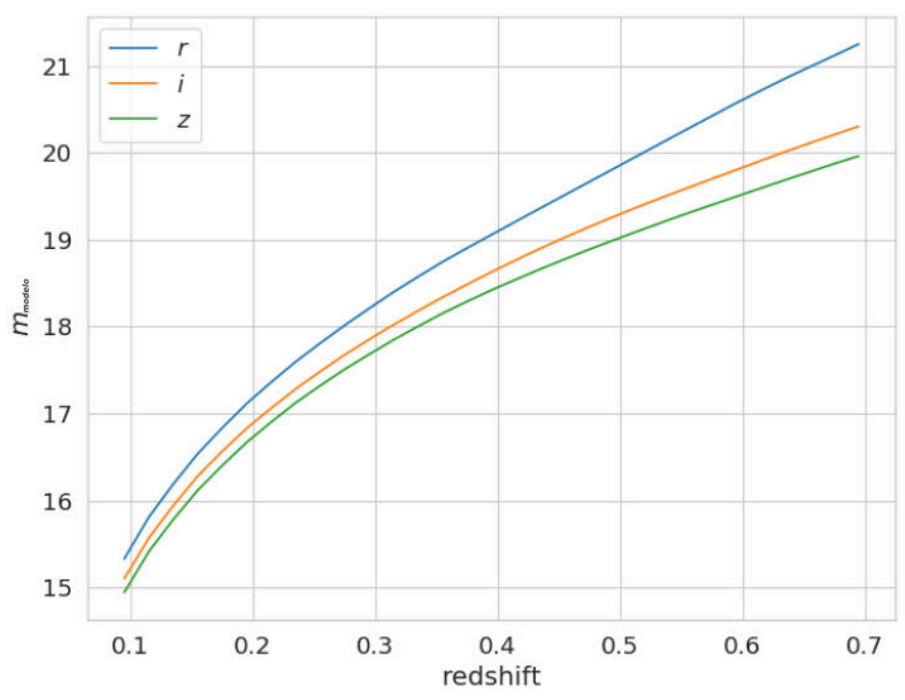

Figura 4.1: Modelo de magnitude observada ao longo do redshift para uma típica galáxia elíptica para três filtros, $r, i$ e $z$.

empírica, é válida para aglomerados até $z \approx 1$, como alguns estudos sugerem (Mei et al. 2009; Hennig et al., 2017; Nishizawa et al., 2018).

Nesse sentido, utilizamos o maior catálogo de aglomerados atuais baseado na SV, o redMaPPer Y3, para estimar a evolução dos seus parâmetros. Em específico, usamos a amostra com $\lambda>20$ e limitado por volume que contêm 22 mil aglomerados no intervalo até $z=0.95$. Medimos a inclinação da SV em intervalos de redshift de 0.075 para as três cores usadas nesse trabalho.

Os resultados são mostrados na Figura 4.2, Além disso podemos ver a medida para aglomerados individuais em ponto pretos. A evolução da inclinação da SV acontece principalmente devido ao fato das galáxias ricas em metais se tonarem mais vermelhas mais rápido do que as galáxias pobres em metais ao longo da idade (Gladders et al., 1998). Por outro lado, percebemos que esse efeito depende do índice de cor, em especifico para $(r-i)$ e $(i-z)$ a evolução é menos notável do que em $(g-r)$. Essa diferença está relacionada com o fato de que os filtros medem diferentes partes do espectro.

Nesse trabalho o ajuste dessa relação é de grande importância para se definir o conjunto de galáxias azuis e vermelhas. Sendo assim, ajustamos a sequência vermelha com a inclinação fixa e aplicando um corte de magnitude, $m_{\text {modelo }}+3$. No ajuste temos apenas galáxias dentro de $R_{500}$. Os valores da inclinação são os valores da relação em vermelho do Gráfico 4.2. Fixando a inclinação, evitamos que aglomerados de baixa riqueza ou com problemas de fotometria tivesse valores sem sentido físico da SV, por exemplo inclinação 

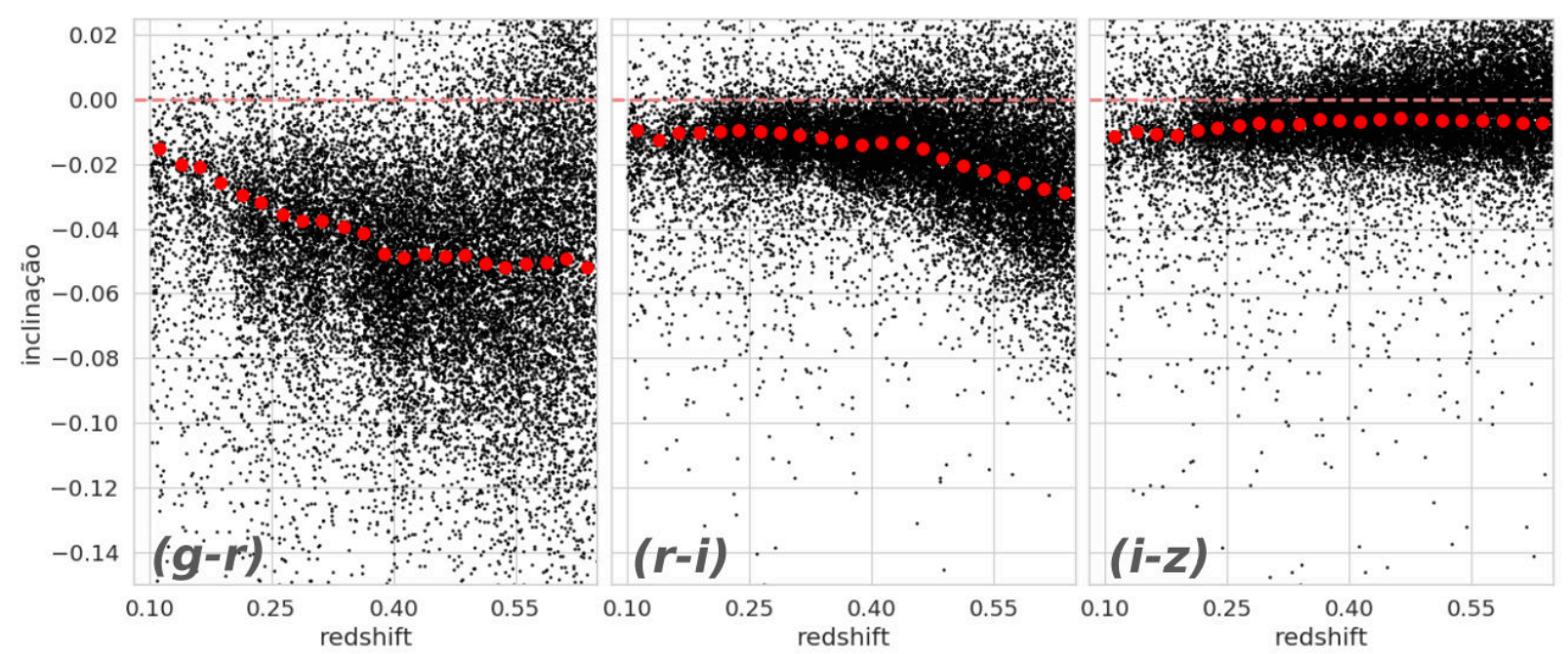

Figura 4.2: Inclinação da sequência vermelha ao longo do redshift para três índices de cor. Em pontos vermelhos temos as medidas em intervalos de redshift de 0.075, e em preto as medidas dos aglomerados individuais. Usamos a amostra de 22 mil aglomerados do redMaPPer Y3 volume limitado com $\lambda>20$.

positiva. Na Figura 4.3 temos um exemplo do ajuste da sequência vermelha para o aglomerado do redMaPPer de MEM_MATCH_ID $=56$ em $z=0.282$.

\subsubsection{Galáxias Azuis e Vermelhas}

Testamos uma modelagem das cores que não leva em conta apenas a sequência vermelhas, mas também a nuvem azul. Para tal usamos método GMM (do inglês, Gaussian Mixture Model), de maneira similar a Hao et al. (2009). O GMM consiste em várias PDFs Gaussianas, em nosso caso, usamos apenas duas distribuições com cor média $\mu_{r, b}$ e variância $\sigma_{r, b}^{2}$, onde os sub-índices $r$ e $b$ referem-se às distribuições das populações vermelha e azul, respectivamente. A distribuição de cor $\Pi(x)$ é dada pela soma das duas distribuições ponderadas por um fator $w_{r, b}$, conforme a equação abaixo:

$$
\Pi(x)=\left(w_{r} p\left(x \mid \mu_{r}, \sigma_{r}^{2}\right)+w_{b} p\left(x \mid \mu_{b}, \sigma_{b}^{2}\right)\right)
$$

As medidas foram feitas para as cores $g-r, r-i, i-z$ dentro de $R_{500}$ e com galáxias de magnitude menor que $m_{\text {modelo }}+3$. Na Figura 4.4 temos uma representação visual do modelo. Nota-se o bom ajuste para a SV e a nuvem azul na cor $(g-r)$, no entanto, para o outro índice cor é evidente que a distribuição da nuvem azul é menos significativa, esse efeito é devido ao fato que diferentes filtros medem diferentes partes do espectro. Nesse sentido o filtro $g$ está mais próximo da região do espectro que predomina a emissão da 


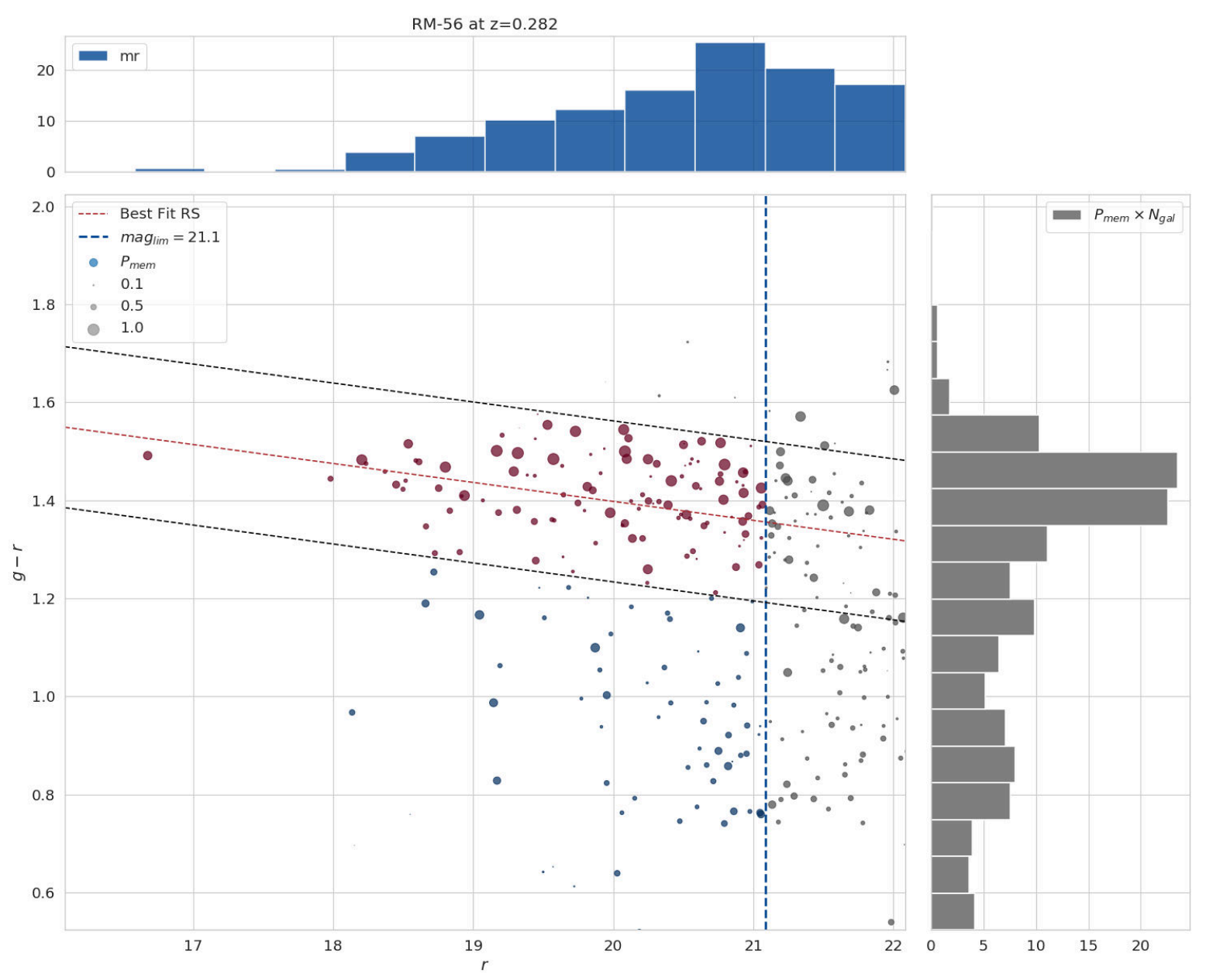

Figura 4.3: Ajuste da sequência vermelha para o aglomerado do redMaPPer de MEM_MATCH_ID $=56$ em $z=0.282$. Observamos todas as galáxias dentro de $R_{500}$. O tamanho dos pontos é proporcional a $P_{m e m}$ da galáxia, escala na legenda. Os pontos em vermelho, azul e cinza são as galáxias da sequência vermelha, nuvem azul e de fundo ou fora do limite de magnitude, respectivamente. A linha tracejada vermelha é o melhor ajuste da SV. A linha tracejada azul é a magnitude limite para esse redshift.

população de estrelas jovens do que da população de estrelas velhas.

De modo similar não conseguimos ajustar a nuvem azul para a maioria dos aglomerados por três grandes fatores. Primeiro, o contraste entre as galáxias azuis e de fundo é baixo. Segundo, a nuvem azul é uma distribuição dispersa. Terceiro, a distribuição de galáxias azuis depende do índice de cor, uma galáxia azul em um índice de cor pode estar na sequência vermelha para um outro índice de cor. Por fim, percebemos que os índices de cor mais próximas do azul são melhores para identificar as galáxias azuis.

Utilizamos o critério usado em outros trabalhos para definir os dois conjuntos de galáxias (Margoniner e de Carvalho, 2000; Loh et al., 2008; Holland et al., 2015; Nishizawa et al., 2018). De maneira que nossa sub-classificação é: 

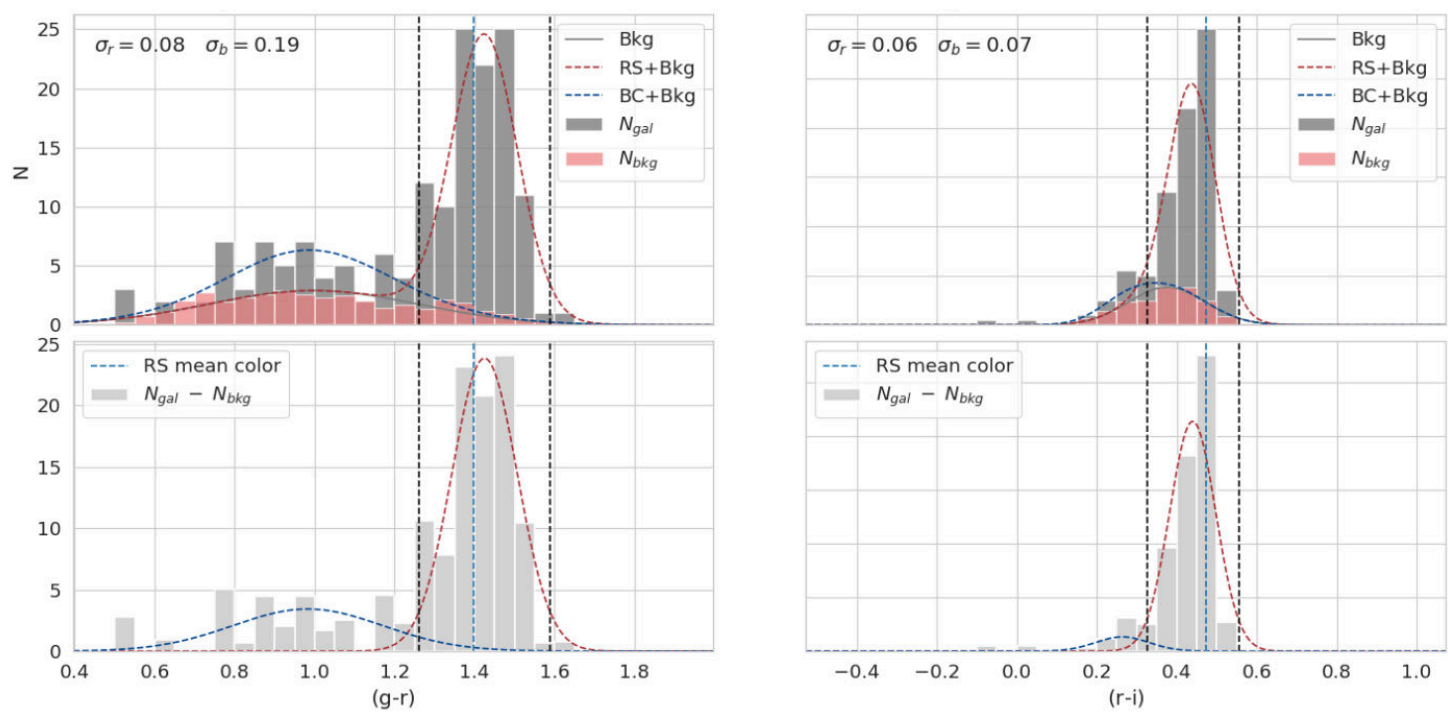

Figura 4.4: Ajuste do modelo Gaussian Mixture Model (GMM) para o aglomerado do redMaPPer de MEM_MATCH_ID $=56$ em $z=0.282$, nas cores $(g-r)$ e $(r-i)$ e para galáxias dentro de $R_{500}$. Parte inferior: temos a distribuição de cor subtraída pela distribuição de galáxias de fundo, As curvas tracejadas vermelhas e azuis correspondem ao ajuste do método GMM para a sequência vermelha (SV) e a nuvem azul, respectivamente. A linha tracejada preta é $\pm 2 \sigma_{r}$ em torno da média da distribuição da SV e a linha azul claro é a cor média derivada da relação do aglomerados da amostra do redMaPPer. Parte superior: em cinza escuro temos a distribuição de galáxias ponderada por $P_{m e m}$, em vermelho claro temos as galáxias de fundo também ponderadas por $P_{m e m}$ multiplicado por um termo de correção da área. A curva cinza é um ajuste gaussiano da galáxias de fundo meramente ilustrativo.

- galáxias vermelhas: objetos dentro $\pm 2 \sigma_{r}$ do ajuste da sequência vermelha.

- galáxias azuis: objetos abaixo de $-2 \sigma_{r}$ do ajuste da sequência vermelha.

- galáxias de fundo: objetos acima de $2 \sigma_{r}$ do ajuste da sequência vermelha.

Na contagem de galáxias vermelhas e azuis subtraímos a contaminação de galáxias de campo ponderada pela razão das áreas.

$$
\eta_{\text {cor }}=\eta_{\text {mem,cor }}-\gamma \times \eta_{\text {bkg,cor }}, \quad \text { cor }=\text { azul, vermelha } .
$$

Onde $\eta_{\text {cor }}$ é a soma das galáxias vermelhas ou azuis ponderadas pela probabilidade de ser membro abaixo do corte de magnitude, $m_{\text {modelo }}+3$. Os sub-índices mem e bkg denotam galáxias na região do aglomerado e no anel de fundo, onde $\gamma$ é a razão das áreas. A contribuição das galáxias de campo foi feita no anel de $(4-6) \times R_{500}$. 
Capítulo 5

\section{Evolução de Galáxias em Aglomerados}

O ambiente denso de aglomerados de galáxias influencia a evolução das galáxias de diversas maneiras. Um dos fenômenos mais evidente é a segregação morfológica, notada desde os primórdios da Astronomia Extragaláctica por Hubble e Humason (1931) . Os tipos morfológicos de galáxias tipo joviais, E+S0, constituem $90 \%$ da população galáctica em ambientes mais densos enquanto em regiões de campo sua frequência é por volta de 40\%, mostrado por Dressler (1980) usando uma amostra de 55 aglomerados do universo local. Além do mais, Butcher e Oemler (1984) usando uma amostra de aglomerados até $z<0.5$ mostrou uma evolução na fração de galáxias azuis. Indicando, assim, um fator evolutivo na população galáctica do aglomerados.

Nesta dissertação revisitamos esses dois últimos trabalhos, com métodos distintos, em amostras representativas e num intervalo de redshift mais amplo. Exploramos possíveis dependências desses resultados com o estado dinâmico do aglomerado. Para isso utilizamos dois grandes levantamentos fotométricos profundos. A nossa amostra consistem em 97 aglomerados observados em raios-X pelo Chandra e com fotometria do SDSS e do DES.

\subsection{Relação Cor-Raio}

A relação morfologia densidade pode ser traduzida pela cor da galáxia, pois a maioria das galáxias que compõem a sequência vermelha dos aglomerados são elípticas ou lenticulares. Nesse sentido, definimos a nossa população galáctica em dois conjuntos, vermelhas e azuis, onde as vermelhas (azuis) refletem de certa maneira a morfologia das galáxias tipo joviais (tardios). Além disso, a seleção da cor pode influenciar na população escolhida, sendo assim discutimos o método que melhor traça o tipo morfológico em nossa amostra. 


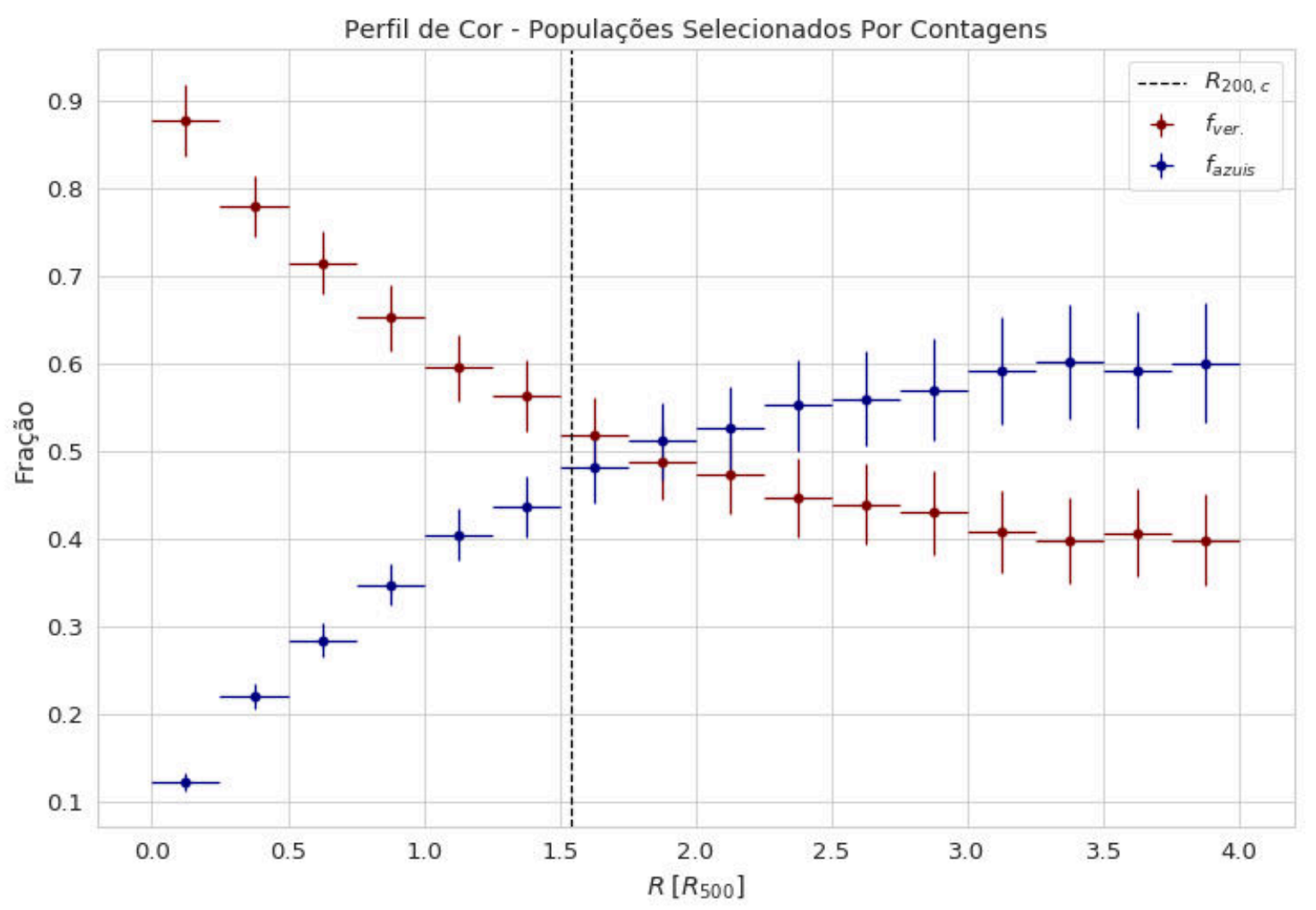

Figura 5.1: Perfil da fração de galaxias azuis e vermelhas em função do raio normalizado por $R_{500}$ para 14 aglomerados em redshift intermediário, $z \in(0.35,0.50)$, na amostra XMATCHA-DES. Os erros mostrados são Poissonianos.

No Gráfico 5.1 temos que a fração de galáxias vermelhas é $87 \%$ na região central, a fração de galáxias azuis se iguala por volta de $2 \times R_{500}$, e chega a valores típico de campo nas regiões mais externas. Em plena concordância com os valores de maior densidade e menor densidade de galáxias encontrados em Dressler et al. (1997) que estudaram a relação nesse mesmo intervalo de redshift.

Os trabalhos de Dressler (1980); Dressler et al. (1997) não utilizaram o raio projetado, utilizaram a densidade local, $\Sigma$, argumentaram que a segregação morfológica seria um efeito local. Por outro lado, com o advento de amostras em raios-X, Whitmore et al. (1993) defenderam a relação morfologia-raio, ou seja, um efeito global. Para isso, esses autores revisitaram o trabalho de Dressler et al. (1985) com os mesmos 55 aglomerados, e as mais ou menos as mesmas 6.000 galáxias. E mostraram que a relação existia e era robusta variando propriedades como riqueza, luminosidade em raios-X, $L_{X}$, ou dispersão de velocidade, $\sigma_{c l s}$. Neste trabalho nos atemos ao cenário em que a segregação morfológica é 
resultado de um efeito global, dependente do raio, ou seja, as galáxias devem "conhecer"sua distância do centro do aglomerado.

Comparamos nossos resultados com os dados de Whitmore et al. (1993), apesar da diferença de redshift das duas amostras, a evolução na população de galáxias não é tão distinta dado que o aumento da fração de E+S0 é menor que a incerteza da medida em seu valor mais central (veja a Figura 1.7).

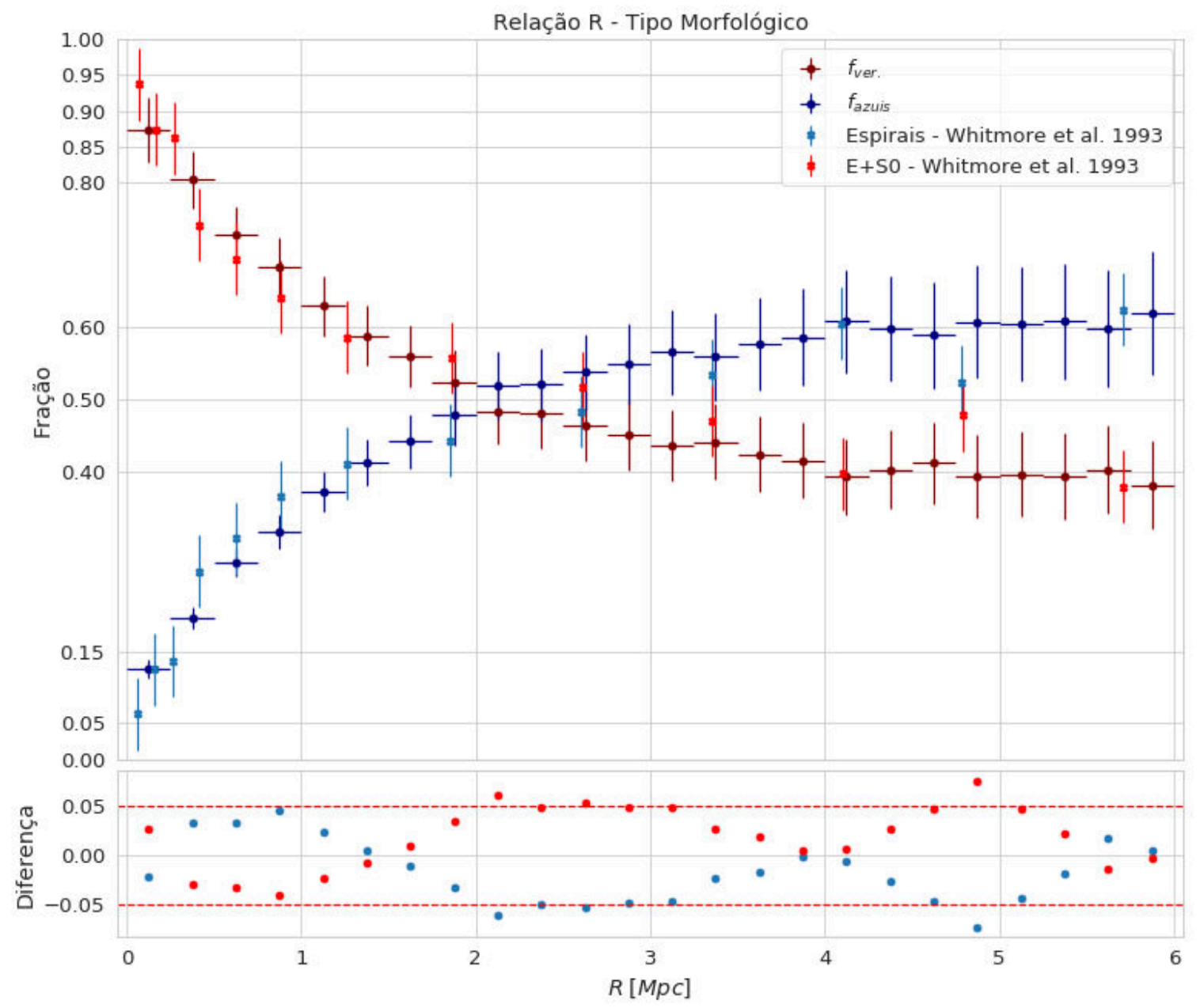

Figura 5.2: Comparação do perfil de fração de galaxias azuis e vermelhas com o tipo morfológico em função do raio em unidades de $M p c$. Usamos a mesma amostra da figura 5.1. As curvas com o tipo morfológico são dos 55 aglomerados e 6000 galáxias do universo local de Whitmore et al. (1993), utilizamos um sigma padrão de 0.05 a fim de comparação. O gráfico abaixo mostra a diferença da curvas em relação ao nosso trabalho. Os resultados concordam em $5 \%$ para quase todos os pontos.

Na figura 5.2 temos uma boa concordância, as duas curvas são compatíveis dentro de $5 \%$, um valor médio de $\sigma$. Temos uma diferença de $5 \%$ a mais de $\mathrm{E}+\mathrm{S} 0$ no valor mais central em concordância com Dressler et al. (1997) que aponta que a fração de E+S0 é apro- 
ximadamente $5 \%$ menor na região mais densa para aglomerados em redshift intermediário. Também podemos perceber uma queda mais abrupta da fração de galáxias espirais do que a fração de galáxias azuis, mais nada além de $1 \sigma$.

Lembrando que em ambos os casos aplicamos um corte de magnitude dependente do redshift. Essa escolha é justificada pelo fato de uma dada uma galáxia com $m_{r}=24.08$ no universo atual - i.e. no limite do corte de magnitude do DES - ter sido menos brilhante no passado devido à formação estelar. Então, aplicando um corte de magnitude constante, não estaríamos comparando essa galáxia com sua equivalente no passado Andreon e Quintana 2006). A magnitude limite utilizada foi $m_{\text {modelo }}(z)+3$ conforme mostrada na seção 4.2.1.

No geral, concluímos que as cores das galáxias são um bom indicador da morfologia. No entanto, durante o nosso estudo percebemos que a seleção das galáxias na sequência vermelha depende da cor utilizada. Nesse sentido, fazemos uma discussão dos efeitos de seleção na seção 5.4 .

\subsection{Evolução: Efeito Bucher-Oemler}

A distribuição e propriedades de Aglomerados de Galáxias ao longo do redshift é uma ferramenta importante para impor vínculos nas teorias de formação de estrutura e modelos cosmológicos. Um aumento gradual da fração de galáxias azuis no intervalo $0.1<z<0.4$, encontrado por Butcher e Oemler (1978, 1984), foi a primeira evidência de evolução de galáxias em ambiente densos. Posteriormente foi confirmado fotometricamente por uma série de estudos (p. ex.: Rakos e Schombert, 1995; Margoniner e de Carvalho, 2000; Goto et al. 2003). Mas este fenômeno foi debatido desde o começo, e com o aumento de estudos sobre a questão se tornou claro que o efeito não é somente uma tendência evolutiva, pois, há um viés de seleção (Andreon et al., 2004). Esse viés pode ser da amostra, ou dos critérios adotados ao se definir a população de galáxias azuis.

Em nosso trabalho, estudamos o efeito Butcher-Oemler ao longo de um largo intervalo de redshift, $0.1 \leq z \leq 0.65$, veja Figura 5.3 . Encontramos o efeito exatamente no intervalo de redshift do estudo clássico, porém, após $z>0.4$ a tendência permanece constante e depois um crescimento não significativo em $z=0.6$.

Esse evolução pode ser real, ou pode ser simplesmente um efeito de seleção. Destacamos dois possíveis vieses de seleção: a amostra de aglomerados selecionados pelo redMaPPer; 


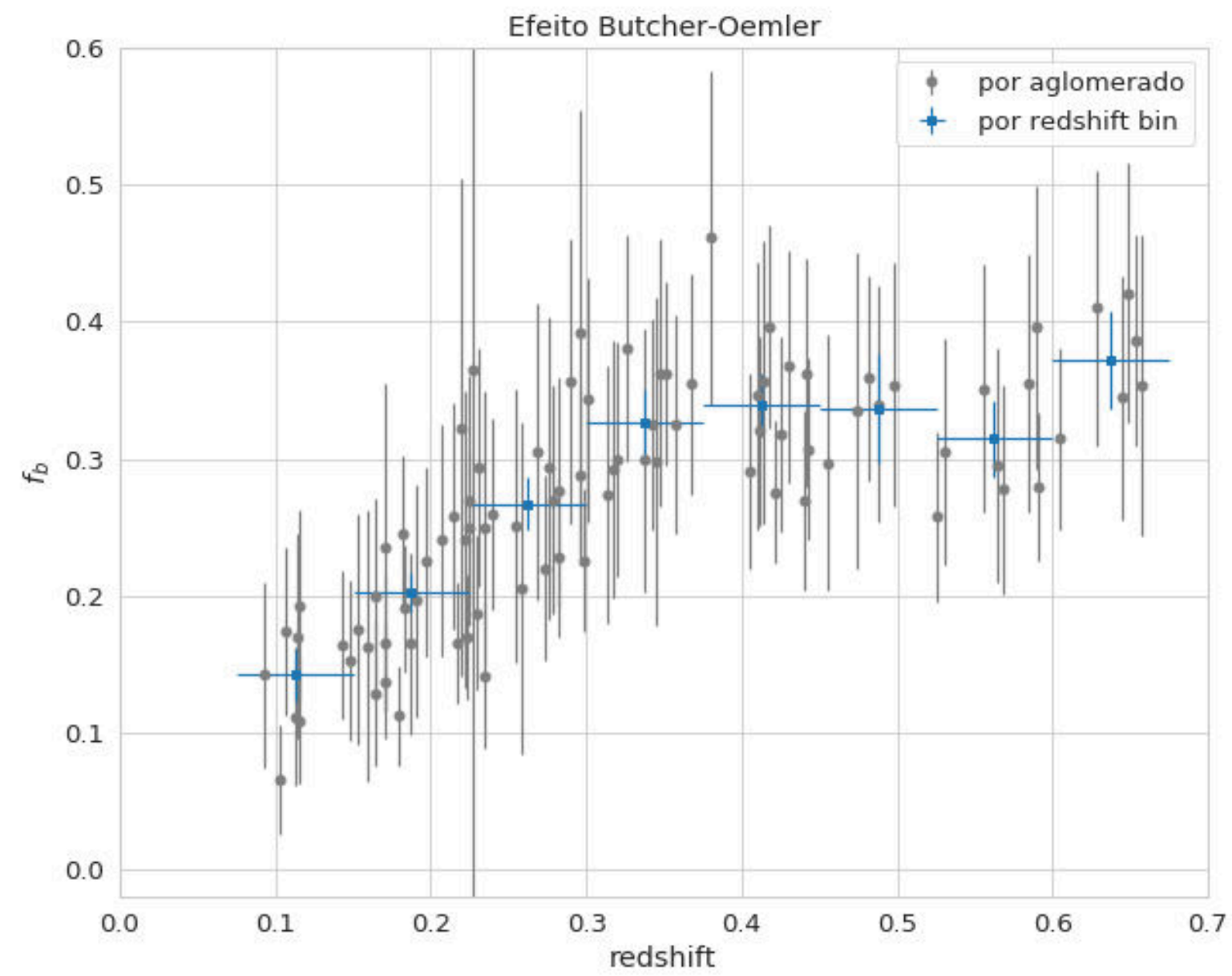

Figura 5.3: Evolução Fração de galaxias azuis selecionadas pela cores $(g-r)$ e $(r-i)$. Os pontos em cinza são as medidas para cada aglomerado, enquanto os quadrados azuis são as medidas em intervalos de redshift - com espessura de 0.075 .

um viés das estimativas de redshift fotométrico do DES por volta de $z \approx 0.4$ (Palmese et al., 2016). Esse viés de redshift está relacionado com a falta de filtros no ultravioleta sendo que após esse redshift o filtro $g$ deixa de ser sensível a quebra de $4000 \AA$ do espectro galáctico.

\subsection{Correlações: Estado Dinâmico}

Escolhemos uma amostra de aglomerados relaxados, vide 2.5.1, no intervalo de redshift, $0.35<z<0.65$, em que não há evolução da fração de galáxias azuis, $f_{b}$. Buscamos estudar as correlações de $f_{b}$ com as propriedades do meio, como massa, temperatura, $Y_{X}$ (indicador de pressão) e estado dinâmico.

Na Figura 5.4 encontramos uma tendência da massa e da temperatura com a fração de 

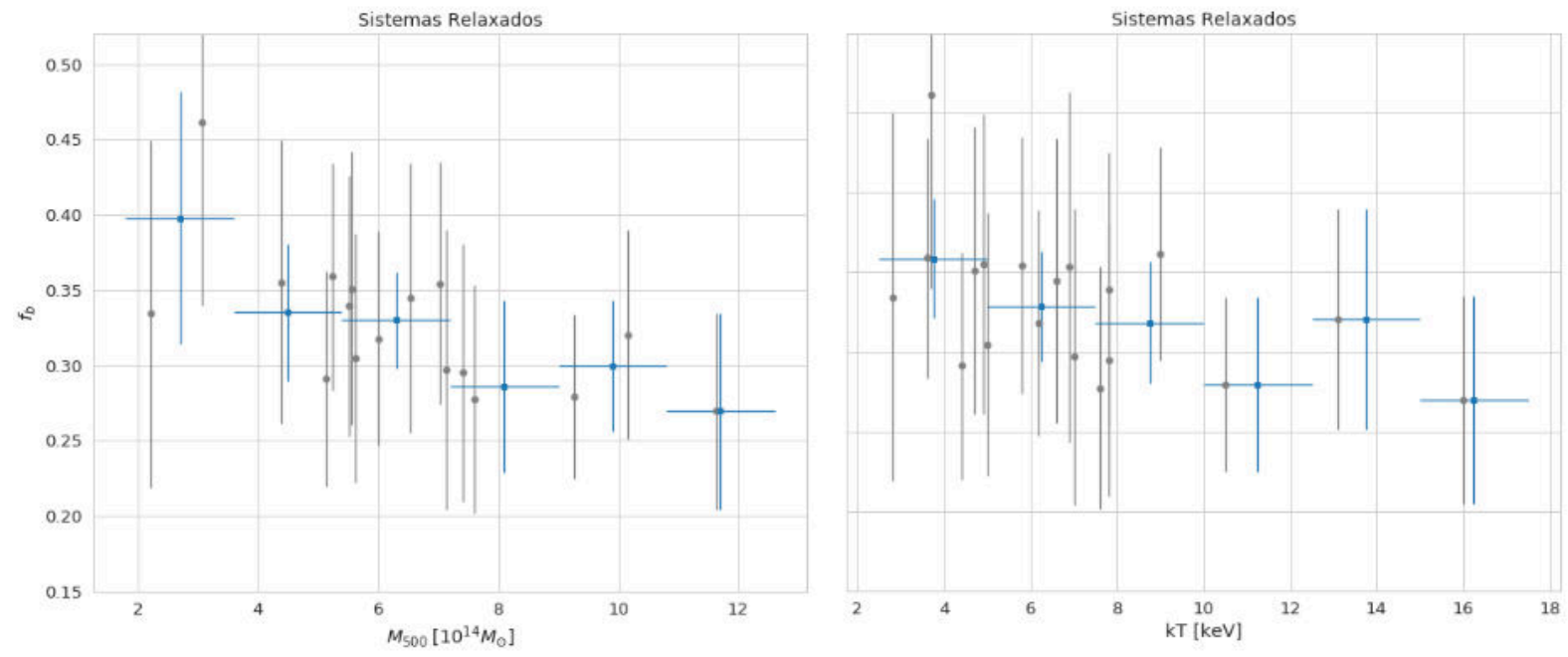

Figura 5.4: Fração de galaxias azuis, $f_{b}$, em função das propriedades físicas do aglomerado, $M_{500}$ e $k T$, para a amostra em redshift intermediário, $0.35<z<0.65$. Os pontos em cinza são as medidas para cada aglomerado, enquanto os quadrados azuis são as medidas em intervalos de massa e temperatura.

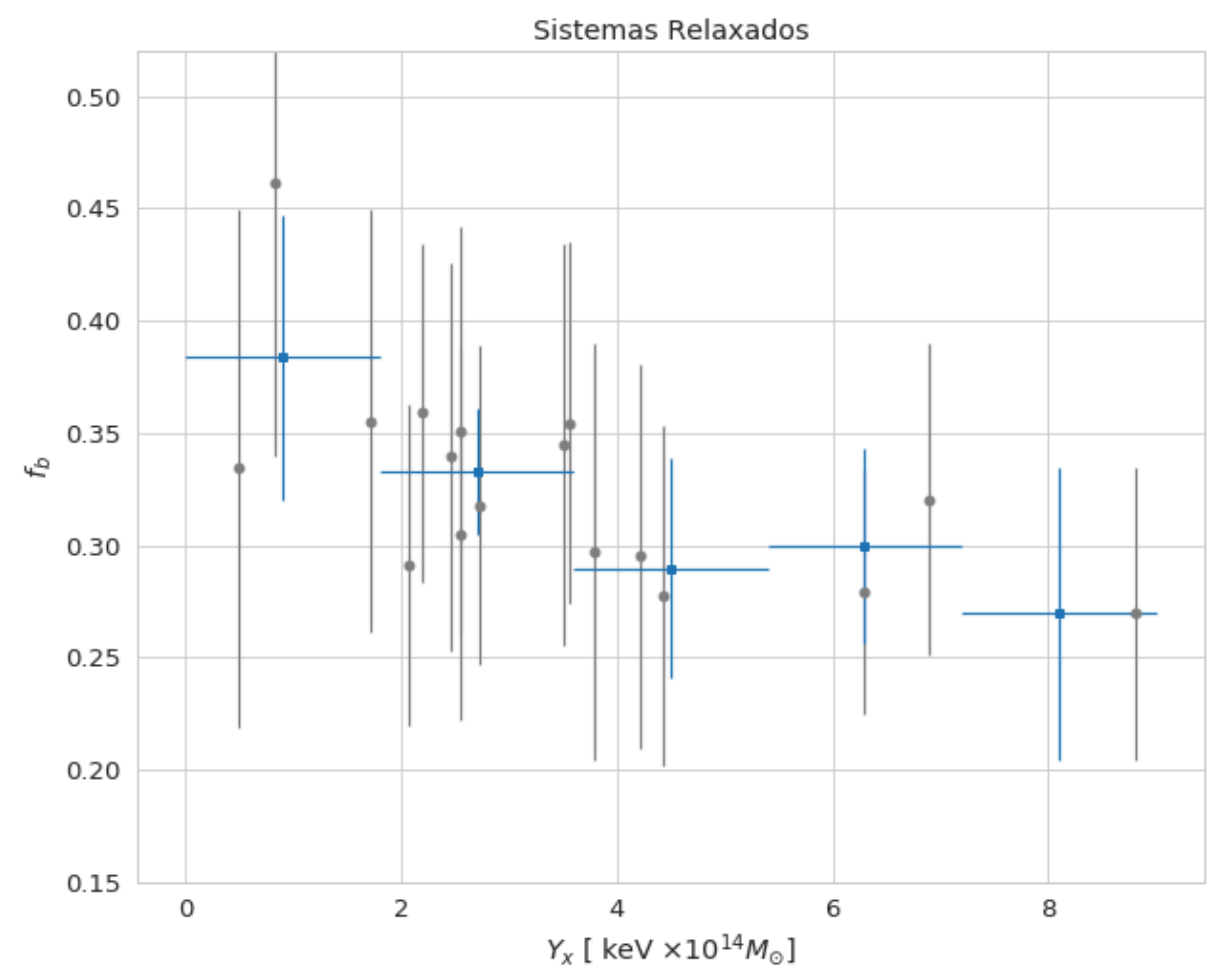

Figura 5.5: Fração de galaxias azuis, $f_{b}$, em função $Y_{X}$, para a amostra em redshift intermediário, $0.35<z<0.65$. Os pontos em cinza são as medidas para cada aglomerado, enquanto os quadrados azuis são as medidas em intervalos.

galáxias azuis. Sendo assim, em aglomerados de maior massa e maior temperatura existem uma maior fração de galaxias vermelhas. No entanto, essas duas quantidades físicas estão 
de certa forma ligadas, pois, a temperatura é um indicativo da massa. Diferentes medidas observacionais em astronomias refletem uma lei mais fundamental em certos casos, vide o plano fundamental das elípticas. Em nosso caso, o fenômeno por trás do efeito ButcherOemler é a pressão de arraste, então, um indicador de pressão deve ser a causa do fenômeno.

De fato, na Figura 5.5 também temos uma tendência entre $Y_{X}$ e $f_{b}$. Além disso, utilizamos o coeficiente de correlação $\rho$ e o valor-p para verificar a significância estatística dessas tendências. Na Tabela 5.1 temos os valores desses índices estatísticos. Visto que o valor-p abaixo de 0.05 indica uma significância estatística, encontramos que essas tendências são significativas e todas estão anti correlacionadas com a $f_{b}$ segundo o índice $\rho$.

Tabela 5.1 - Teste Estatístico de Correlação

\begin{tabular}{l|lllll}
\hline$f_{\text {azuis }}$ & $Y_{X}$ & $T_{X}$ & $M_{X, 500}$ & $c_{S B}$ & $\langle w\rangle$ \\
\hline$\rho$ & -0.56 & -0.53 & -0.57 & -0.27 & 0.36 \\
valor-p & 0.0203 & 0.0213 & 0.0166 & 0.2698 & 0.1648 \\
\hline \multicolumn{4}{c}{ Nota: amostra em intervalo de redshift $0.35<z<0.65$}
\end{tabular}

Do ponto de vista do estado dinâmico, exploramos possíveis correlações do estado dinâmico do ICM com a população galáctica do aglomerado. Vemos que $f_{b}$ possui uma anticorrelação com $c_{S B}$ e um correlação com $\langle w\rangle$, segundo o indicador $\rho$, mas essas correlações não são significativas segundo o valor-p. Porém antigas classificações morfológicas de aglomerados no óptico mostram diferenças na relação morfologia-densidade (Dressler, 1980) que está ligada com a fração de galáxias azuis. Esse desacordo aponta que a morfologia do Aglomerados no óptico não está necessariamente relacionada ao estado dinâmico do ICM.

Por outro lado, em relação as propriedades físicas do aglomerado, é possível que $f_{b}$ esteja anti-correlacionado com $Y_{X}, T_{X}$ e $M_{X, 500}$. Nesse sentido, dado que esses três parâmetros são no fundo consequências de uma mesma causa, a interpretação física é que a fração de galáxias azuis é menor para aglomerados que exercem uma pressão global maior sobre suas galáxias. Em outras palavras, aglomerados de maior massa são mais eficientes em arrancar o reservatório de gás de galáxias espirais em queda.

Assumindo que essas correlações de fato existam, temos uma evidência forte em favor do cenário em que a pressão de arraste é responsável pela diminuição das fração de galáxias azuis. Porém, não afirmarmos categoricamente essa correlação, é necessário uma investigação em uma amostra significativa, sobretudo em valores de pressão mais alto. 


\subsection{Efeito de Seleção: Escolha da Cor e Espessura da Sequência Vermelha}

Nesta seção é feita uma discussão dos efeitos de seleção. Fazemos uma análise de quanto a escolha do índice de cor e a espessura da sequência vermelha influenciam nas estimativas realizadas neste Capitulo.

Percebemos ao longo dessa análise que a relação morfologia(cor)-raio e o efeito ButcherOemler depende da escolha da cor. Na figura 5.6 verificamos que $f_{b}$ é enviesado pela escolha do índice de cor. Temos que os índices de cor mais próximos do ultravioleta estimam um número maior de galáxias azuis. Por conseguinte, índices de cor mais próximos do infravermelho super estimam a fração de galáxias vermelhas. De fato, Nishizawa et al. (2018) encontraram que a fração de galáxias na sequência vermelha é maior em índices de cores mais próximas do infravermelho.

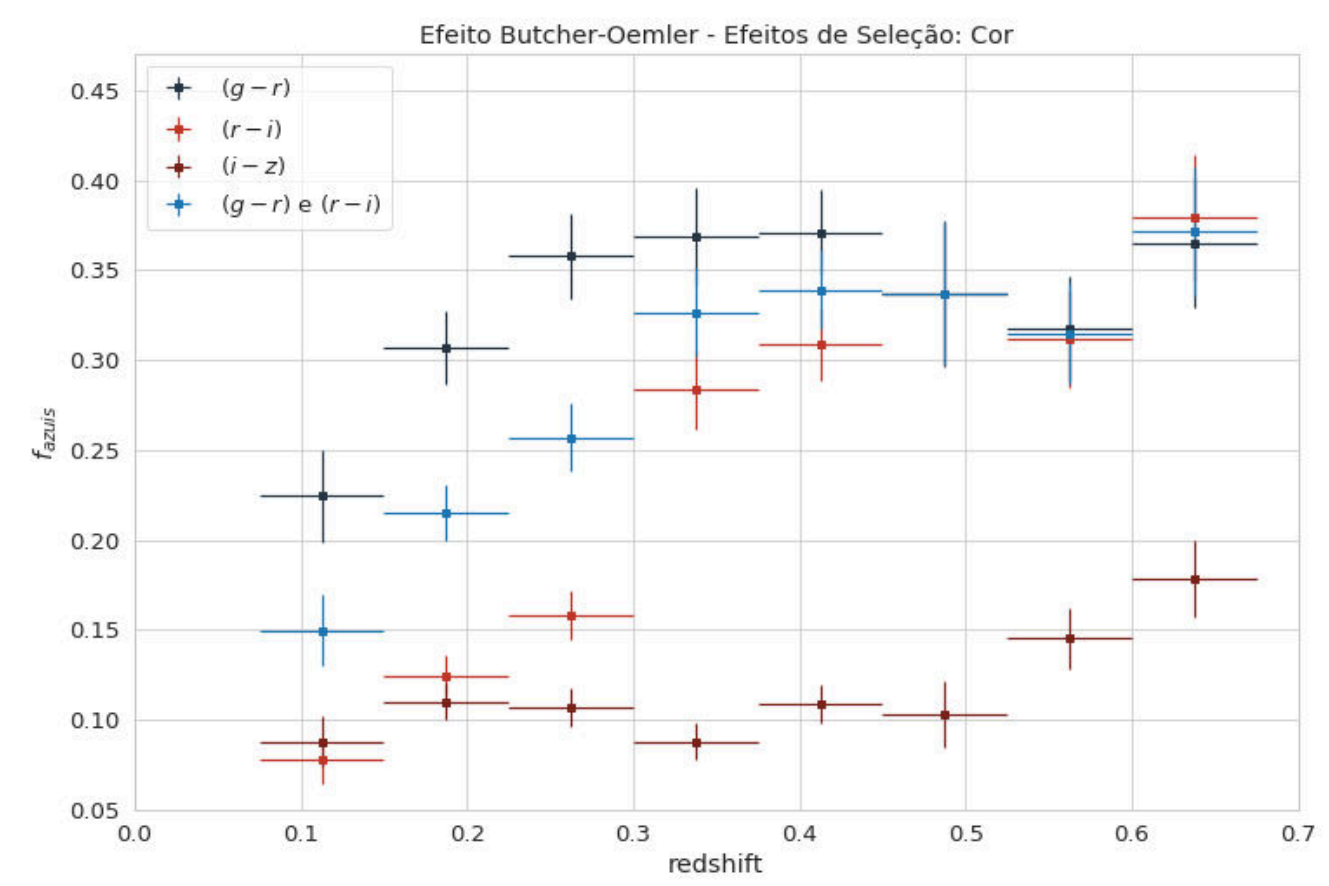

Figura 5.6: Comparação da fração de galaxias azuis ao longo do redshift para diferentes seleções de cor.

No Gráfico 5.7 temos a comparação dos perfil radial de $f_{v e r m}$. utilizando os três índices de cor usados nesse trabalho para a amostra de redshift intermediário, além das combinações $(g-r)$ e $(r-i)$. Vemos que as cores estão em acordo razoável com os tipos 
morfológicos, exceto para cor $(i-z)$ que falha miseravelmente em traçar a fração de azuis. Notamos que $(g-r)$ sub estima e $(r-i)$ super estima as vermelhas em $10 \%$ nas regiões mais externas, enquanto a combinação dessas duas cores atinge a fração de $40 \%$ típica dessas galáxias em regiões de campo. Em suma, a combinação de duas cores para a seleção dos grupos vermelhos e azuis vermelhos é uma técnica ainda não utilizada em trabalhos dessa natureza, mas que se mostra efetiva para traçar a relação morfologia(cor)-raio.

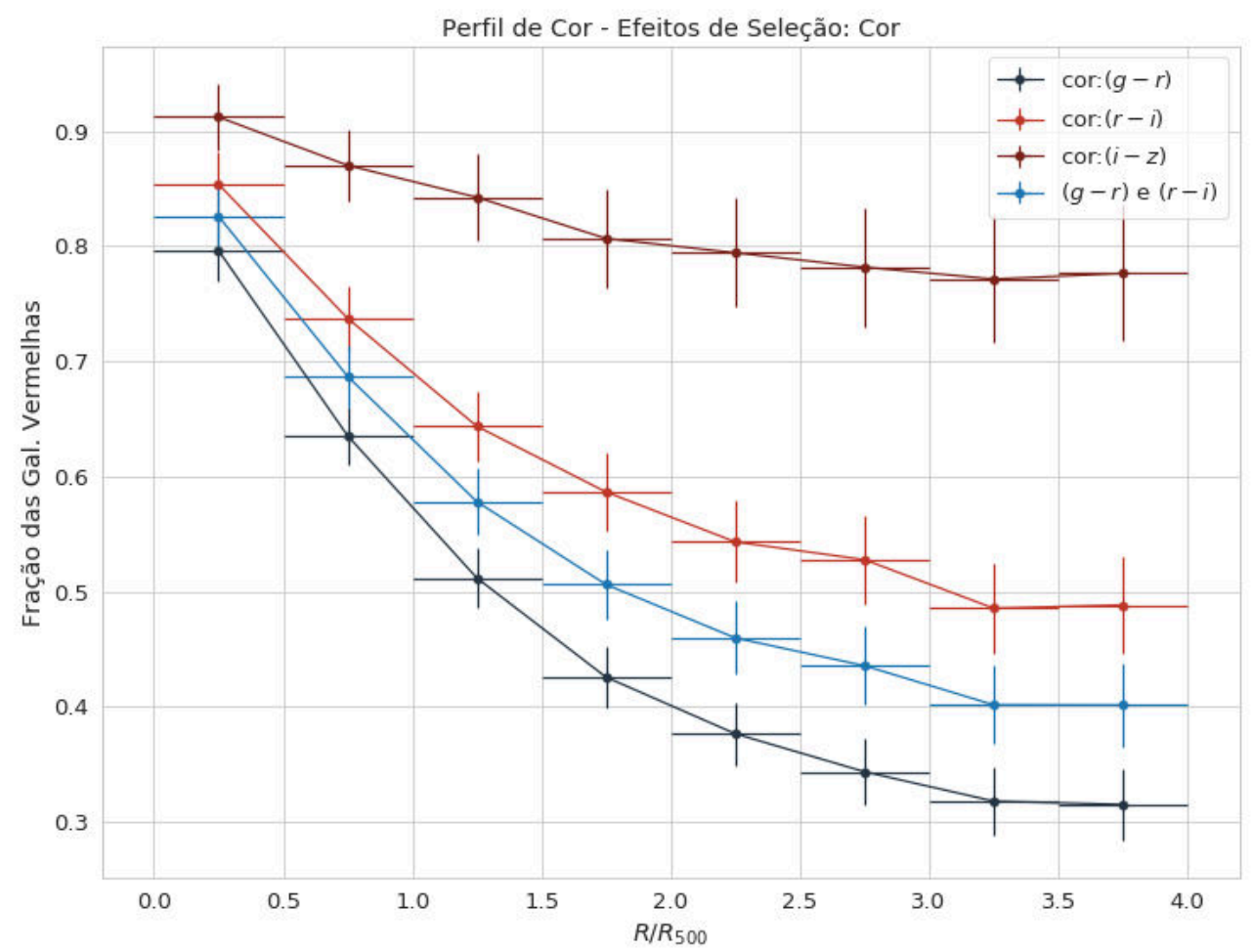

Figura 5.7: Comparação da relação morfologia(cor)-raio para diferentes seleções de cor.

A espessura da sequência vermelha é um parâmetro crucial para se definir o conjunto de galáxias vermelhas e azuis. Não utilizamos uma espessura fixa para a sequência vermelha. Como vimos na Seção 4.1, esse valor depende do índice de cor e do redshift. Uma consequência da formação estelar. De modo que o índice de cor de uma galáxia espiral tem uma evolução diferente de uma galáxia elíptica ao longo do redshift, de maneira que não é possível definir uma classe de galáxias azuis e vermelhas com uma mesma espessura (Andreon e Quintana, 2006).

Sendo assim, realizamos um análise comparativa. Estimamos a fração de galáxias azuis $\left(f_{b, 0.20 \mathrm{mag}}\right)$ a uma espessura fixa de $0.20 \mathrm{mag}$ na cor $(g-r)$, mesmo procedimento usado 


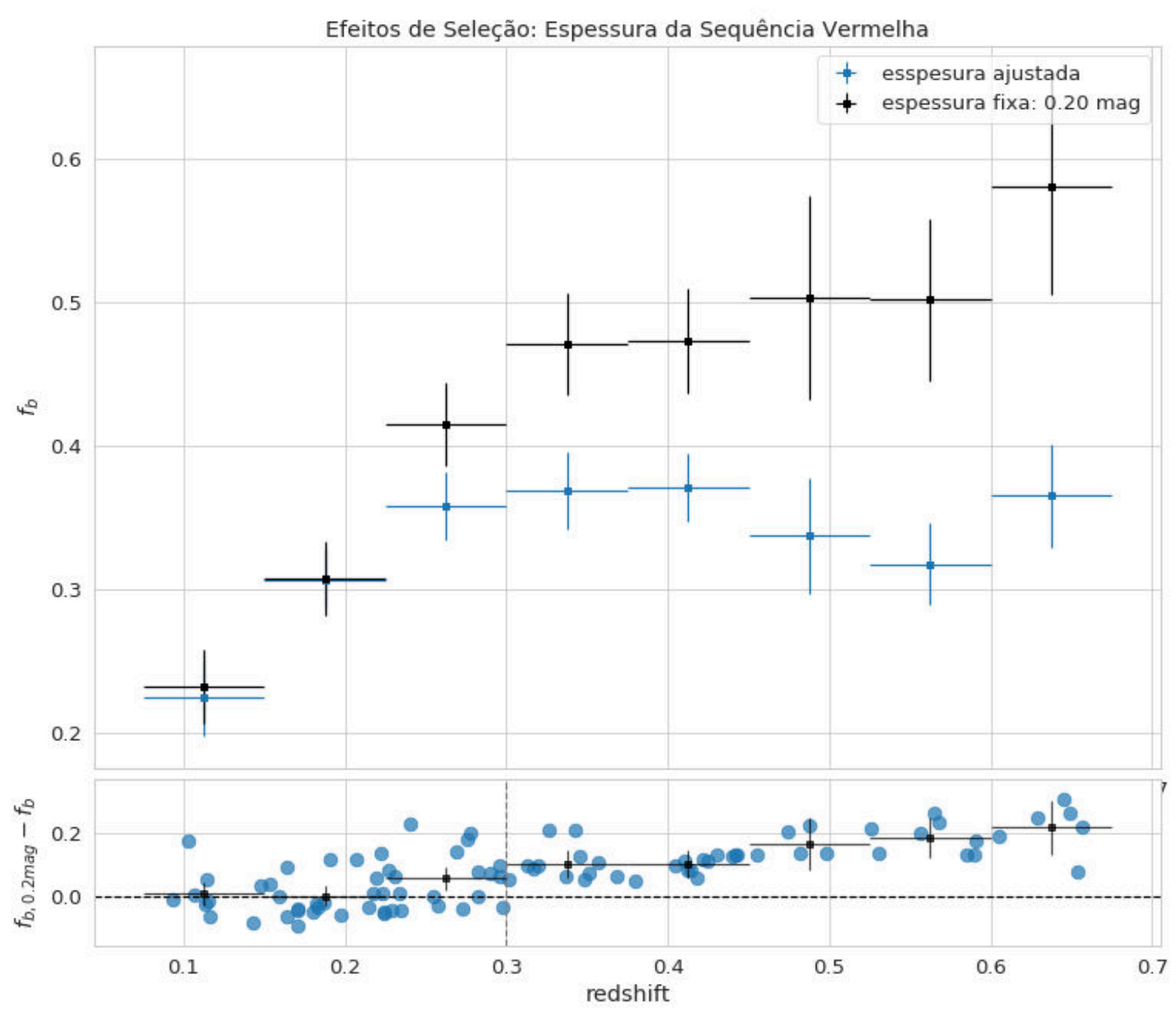

Figura 5.8: Comparação da fração de galaxias azuis selecionadas pela cor $(g-r)$ para duas espessuras da sequência vermelha, fixa em 0.20 mag em preto, e ajustada em azul claro (veja seção 4.1). Parte superior: os pontos se referem aos valores de $f_{b}$ calculados para intervalos de redshift de 0.075. Parte inferior: temos a diferença entre $f_{b}$, em azul claro para cada aglomerado, em preto a diferença em intervalos de redshift.

em Margoniner e de Carvalho (2000). Comparamos $f_{b, 0.20 m a g}$ com os nossos valores de $f_{b}$, na cor $(g-r)$. No Gráfico 5.8 encontramos um viés significativo para aglomerados acima de $z>0.2$. No intervalo de redshift mais próximo, obtivemos um acordo dentro de $8 \% \mathrm{e}$ depois uma diferença crescente que chega a ser de $20 \%$.

Esse viés mostra que métodos que utilizam espessura fixa super estimam o efeito Butcher-Oemler. Esse resultado comprovam os argumentos feitos por Andreon e Quin$\tan (2006)$.

Portanto, a escolha do índice de cor e da espessura da sequência vermelha são fatores 
de seleção importantes em nosso trabalho. Mostramos que a fração de galáxias azuis é super estimada quando calculada usando uma espessura fixa, principalmente para $z>0.2$. A combinação de dois índices de cor traça melhor os tipos morfológicos das galáxias do que quando usado apenas um. Em nosso trabalho os índices de cor apropriados para o intervalo de redshift em questão são $(g-r)$ e $(r-i)$, no caso, de redshift mais altos é interessante adicionar o cor $(i-z)$. 
Capítulo 6

\section{AGNs em Aglomerados de Galáxias}

Galáxias de núcleo ativo (AGNs) são uma fase importante da evolução de galáxias massivas. Dado que o paradigma atual é que todas galáxias massivas possuem um buraco negro supermassivo central (BNS) e que este tem uma fase de acreção. Mais ainda, a fase nuclear é considerada um importante mecanismo de regulação da formação estelar e na transformação de galáxias azuis (formadoras de estrelas) em vermelhas, galáxias passivas (p. ex.: Hopkins et al., 2008; Hickox et al., 2009).

O abastecimento da atividade nuclear depende do reservatório de gás frio da galáxia e da eficiência do mecanismo de transporte do gás até a região central, onde o BNS se localiza. Qualquer mecanismos que exerça um torque sobre o gás em direção central pode em princípio gerar a atividade nuclear. Acredita-se que instabilidades de barra (Hopkins et al., 2008) e interações de galáxias são mecanismos dominantes, e recentemente, pressão de arraste (Marshall et al., 2018) também foi invocado no contexto de aglomerados.

AGNs em aglomerados são mais raras em comparação com o campo. Apesar da dependência de AGNs ser afetada pelo ambiente ainda ser debatida, vários autores defendem essa tese (p. ex.: Kauffmann et al., 2004; Gilmour et al., 2007; Ehlert et al., 2015; Lopes et al., 2017; Gordon et al., 2018), alguns com menos afinco, pois possuem evidências marginais para tal conexão (Man et al., 2019), enquanto outros estudos que fizeram a seleção de AGNs em raios-X discordam (Haggard et al., 2010; Koulouridis et al., 2014).

Na discussão Nature versus Nurture em relação à atividade nuclear, consideremos por ora, os efeitos ambientais. Coloca-se que a menor frequência de AGNs em aglomerados é devido a encontros colisionais, seja por fusões ou por assédio galáctico (Ehlert et al., 2015, Koulouridis e Bartalucci, 2019; Man et al., 2019). Outros autores, defendem que a questão seja vista pelo ponto de vista do reservatório de gás. Assim como para galáxias espirais, o 
ambiente hostil do aglomerado inibe a formação estelar através da retirada do gás frio, o mesmo deve acontecer para as galáxias com atividade nuclear, mais ainda, alguns autores apontam para um efeito "Butcher-Oemler das AGNs" (Eastman et al., 2007; Martini et al. 2009; Aird et al., 2012).

Sendo assim, a demografia e a evolução das AGNs são pontos chaves na elucidação dessa discussão. De fato, em relação a demografia, cada fenômeno é mais provável em uma dada região do aglomerado. Por exemplo, a pressão de arraste é mais favorável nas regiões centrais do aglomerado. Enquanto, a colisão de galáxias pode ser mais efetiva em regiões mais externas, ou em regiões bem centrais, na forma de encontros rápidos.

Do ponto de vista da evolução, um aumento da fração de AGNS, $f_{\mathrm{AGNs}}$, ao longo do redshift pode indicar tanto que processos de ambiente são responsáveis pela diminuição da atividade nuclear das galáxias ou que o número de interações galácticas foi mais importante no passado.

Neste capítulo discutimos dois cenários. Primeiro, a pressão de arraste seria o mecanismo responsável pela raridade de AGNs em aglomerados, sendo capaz de diminuir ou até mesmo arrancar o reservatório de gás frio das galáxias hospedeiras, e assim, gerando o fim da formação estelar e atividade nuclear. Segundo, fusão de galáxias pode ser o mecanismo responsável por disparar a atividade nuclear em AGNs. Em particular, um aumento da $f_{\mathrm{AGNs}}$ ao longo do redshift nas regiões exteriores do aglomerado poderia corroborar para tal cenário, pois, segundo o contexto hierárquico, os aglomerados crescem por acreção e este mecanismo foi mais intenso no passado. Lemrando que a fusão de galáxias tem uma dependência com a região do aglomerado, dado que a taxa de fusões de galáxias é bem menor no centro e pode ser considerável na periferia do aglomerado.

Em nosso trabalho, utilizamos um catalogo de AGNs com aproximadamente 1000 objetos em 96 aglomerados, em um intervalo de redshift de 0.1 a 0.65 , conforme mostrado em 2.5.1. Estudamos se as AGNs dependem do meio ou não, se sim, qual é o mecanismo responsável. Fazemos um estudo único correlacionando o estado dinâmico com a frequência dessa população, investigamos se a fração das AGNs é diferente em aglomerados cool-core ou não, e em aglomerados perturbados e relaxados. Além disso, estimamos a evolução da fração desses objetos com o redshift. E discutimos a dependência dessa evolução com a região do aglomerado, e o obscurecimento das galáxias. 


\subsection{A frequência de AGNs Dependem Do Meio}

Conforme discutido anteriormente, a distribuição de AGNs é uma peça importante para elucidar a dependência dessas galáxias com o meio. Nesta seção, apresentamos a distribuição dessa população galáctica de duas formas distintas, em forma de contagens, $N_{A G N s}$, e por fração, $f_{\mathrm{AGNs}}$. Essa escolha tem por fim a comparação com estudos de AGNs selecionadas apenas em raios-X que não encontraram uma supressão de AGNs nas regiões internas.

Em nossa amostra encontramos uma correlação da fração de AGNs com o ambiente. Conforme vemos no Gráfico 6.1, a $f_{\mathrm{AGNs}}$ varia de maneira significativa com o raio projetado, assumindo o valor de $2 \%$ no centro, com uma inclinação acentuada atinge um plateau em $4.5 \%$ entre $1.0-2.5 R_{500}$ e depois cai a $1 \%$ no último anel. Tal comportamento é compatível com resultados encontrados por Lopes et al. (2017) que usando uma amostra de aglomerados no universo local e com AGNs selecionadas por linhas de emissão encontrou encontrou que a $f_{\mathrm{AGNs}}$ é $2 \%$ no centro e tem um pico de $(6 \pm 1) \%$ em $1.5 R_{200}$.

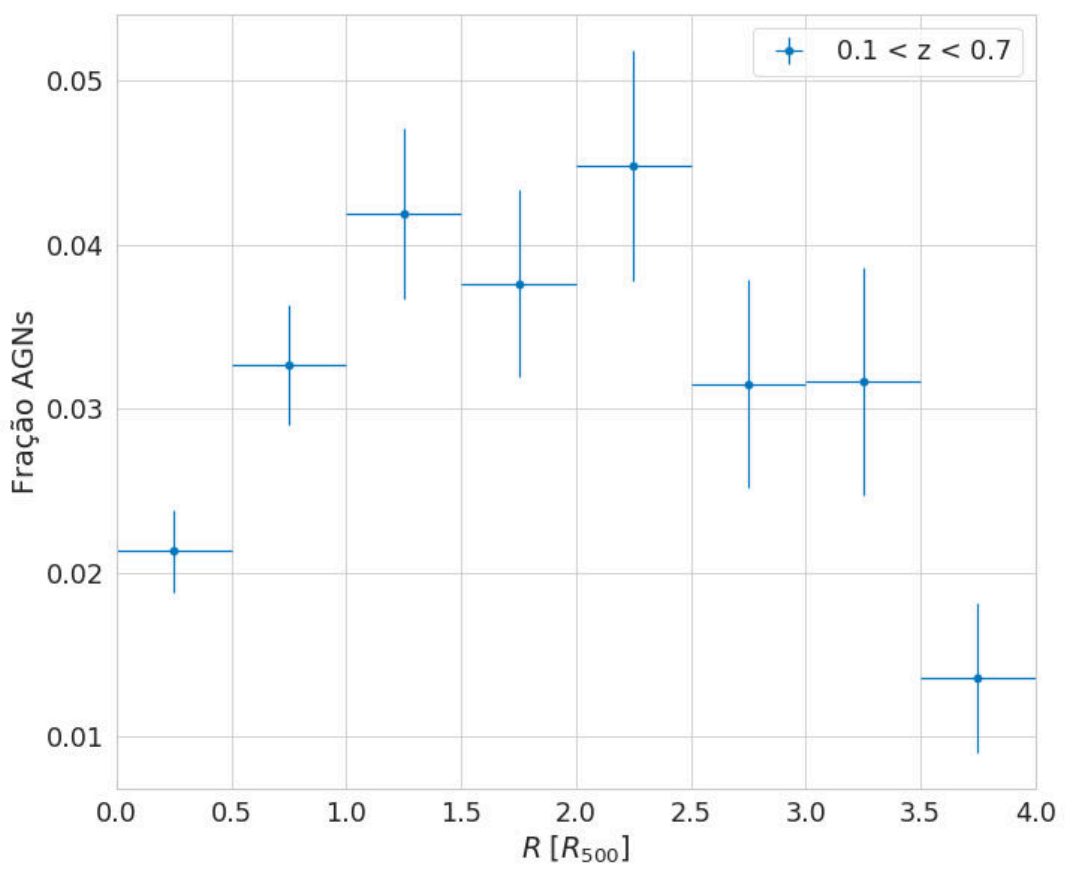

Figura 6.1: Fração de AGNs ao longo do raio projetado do aglomerado em unidades de $R_{500}$. A amostra contém aglomerados um intervalo de redshift de 0.1 a 0.65 e possui 680 AGNs dividas em anéis $0.5 R_{500}$ de espessura e galáxias hospedeiras com magnitudes $M_{r} \leq-19.8$. A fração de AGNs é simplesmente o número desses objetos divido pela riqueza do aglomerado num dado anel, a riqueza é calculado assumindo o mesmo corte de luminosidade. Os erros mostrados são Poissonianos. 
Recentemente, Marshall et al. (2018) realizaram simulações hidrodinâmicas e afirmaram que a pressão de arraste pode induzir a atividade nuclear e o aumento da formação estelar. Após comparações feitas no espaço de fase das simulações com as observações, eles mostraram que um certo regime intermediário de pressão, a distribuição de AGNs no espaço de fase projetado apresenta um pico de densidade na região entre $0.5-1.0 R_{v i r}$ para $z=0$ e $1.0-1.5 R_{\text {vir }}$ para $z=1$.

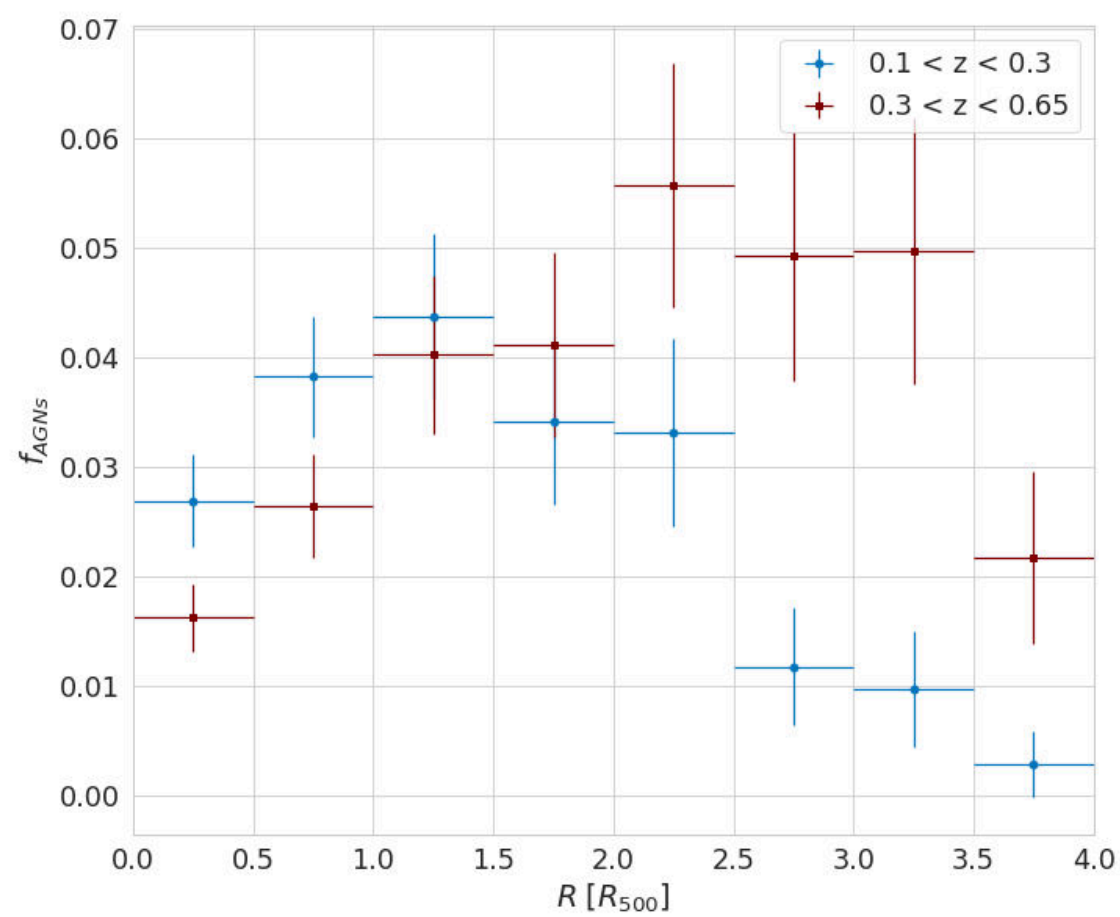

Figura 6.2: Fração de AGNs ao longo do raio projetado do aglomerado em unidades de $R_{500}$. A amostra é divido em dois intervalos de redshift, $0.1<z<0.45$ e $0.45<z<0.65$. Os erros mostrados são Poissonianos.

Os resultados encontrados neste trabalho estão de acordo com esse cenário, dado que o nosso primeiro pico da fração de AGNs é em $1.5 \times R_{500} \approx R_{v i r}$. Além disso, na Figura 6.2 temos a distribuição da $f_{\mathrm{AGNs}}$ ao longo do raio projetado para os intervalos $0.1<z<0.45$ e $0.45<z<0.65$. E podemos ver que existe um deslocamento do pico para $\sim 2.5 R_{500} \approx$ $1.5 R_{200, c}$ no intervalo de redshift mais alto.

No entanto este deslocamento do pico da $f_{\mathrm{AGNs}}$ para regiões mais externas pode indicar um outro processo predominante. Por outro lado, o deslocamento pode ser devido a um efeito de seleção das fontes pontuais em raios-X, considerando que apenas uma observação do Chandra para um aglomerado não garante a completeza da amostra nas regiões mais externas para $z<0.3$. 
Se considerarmos simplesmente o número de AGNs ao longo das regiões do aglomerado, o cenário é outro. Os maiores valores estão na região central e depois uma queda acentuada, como podemos ver no Gráfico 6.3.

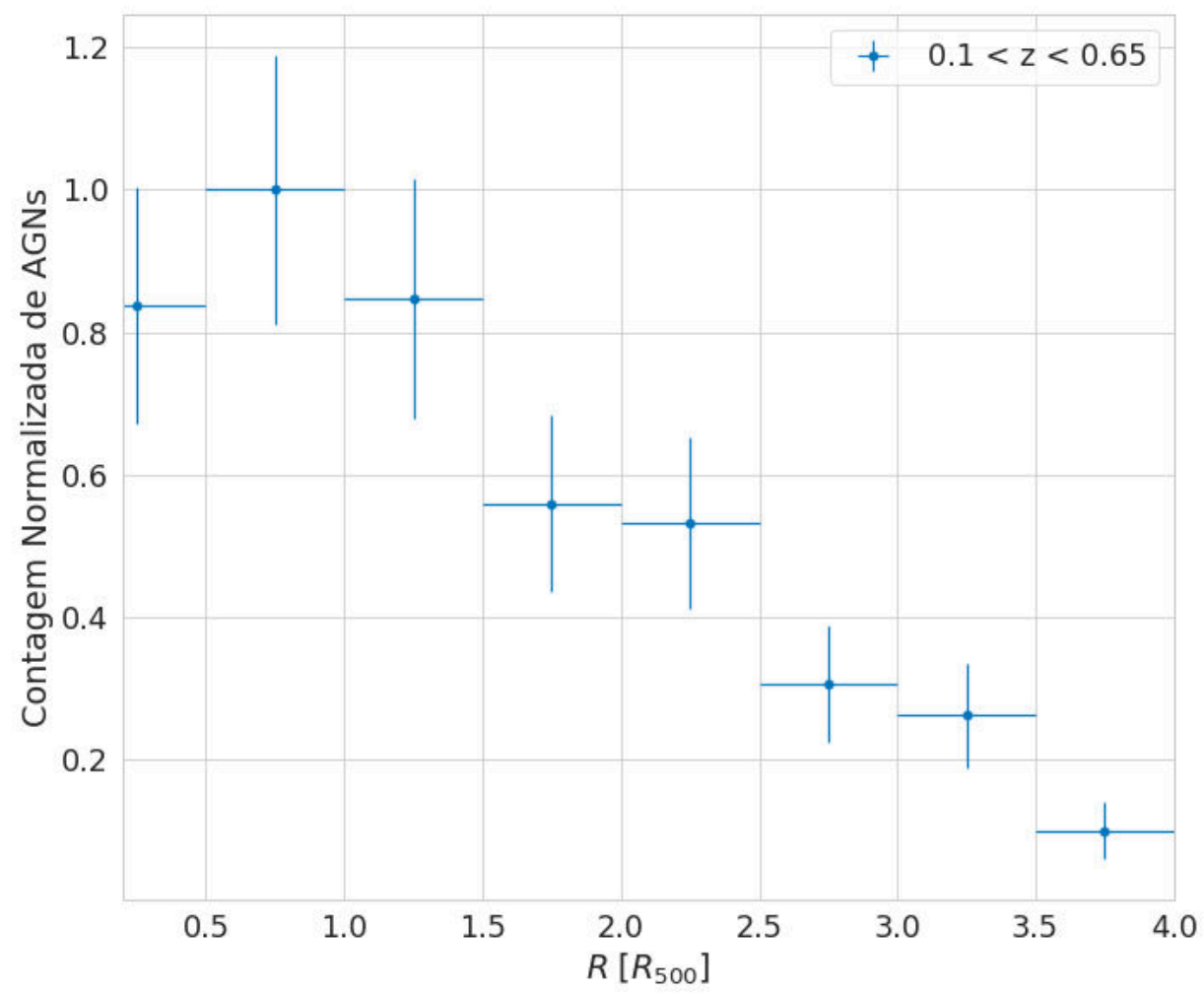

Figura 6.3: Distribuição de AGNs normalizada em um ao longo do raio projetado do aglomerado em unidades de $R_{500}$. A amostra contém aglomerados um intervalo de redshift de 0.1 a 0.65 e possui 680 AGNs dividas em anéis $0.5 R_{500}$ de espessura. Os erros mostrados são Poissonianos.

Koulouridis et al. (2014) utilizando contagens de AGNs encontraram um maior número de fontes dentro de $R_{500}$ e depois uma queda monotônica em acordo com nosso trabalho. Ou seja, essa aparente discordância da correlação de AGNs com o ambiente do aglomerados é fruto de uma interpretação baseado em um indicador enganador, a contagem de AGNs. Sendo as AGNs um subconjunto da população de galáxias, é natural ter um número maior dessas galáxias nas regiões mais centrais do aglomerado. Para ilustrar tal afirmação, peguemos um exemplo: dado um círculo de raio de $1 \mathrm{Mpc}$, em um aglomerado típico teríamos 100 galáxias e 5 AGNs, e numa região de campo, 10 galáxias e uma AGN, digamos. Por conseguinte, ao respondermos a questão onde há uma diminuição de AGNs em aglomerados, se analisarmos apenas a contagem de AGNs, diríamos que não, porém se consideramos a fração de galáxias dentro desse círculo, concluímos que o centro do aglomerado suprime a atividade nuclear. 


\subsection{Efeito Butcher-Oemler das AGNs}

A abundância de gás frio é essencial para a taxa de formação estelar, assim como para a atividade nuclear. Como vimos no capítulo anterior, o aumento da fração de galáxias azuis ao longo do redshift é uma evidência fundamental para efeitos ambientais entre o gás intra-aglomerado e as galáxias. Porém, tal cenário, se sustenta sobre o fato de não existir uma mesma fração de galáxias azuis em aglomerados do que no campo. Feito essas considerações, nos indagamos: existe um efeito Butcher-Oemler das AGNs? Se sim, este é uma evolução da $f_{\mathrm{AGNs}}$ hospedadas por galáxias azuis (AGNs azuis daqui em diante) assim como no efeito clássico? Para estabelecer essa conexão é necessário no mínimo duas evidências: (a) haver uma análogo de segregação morfológica para AGNs; (b) ter uma evolução da fração de AGNs azuis.

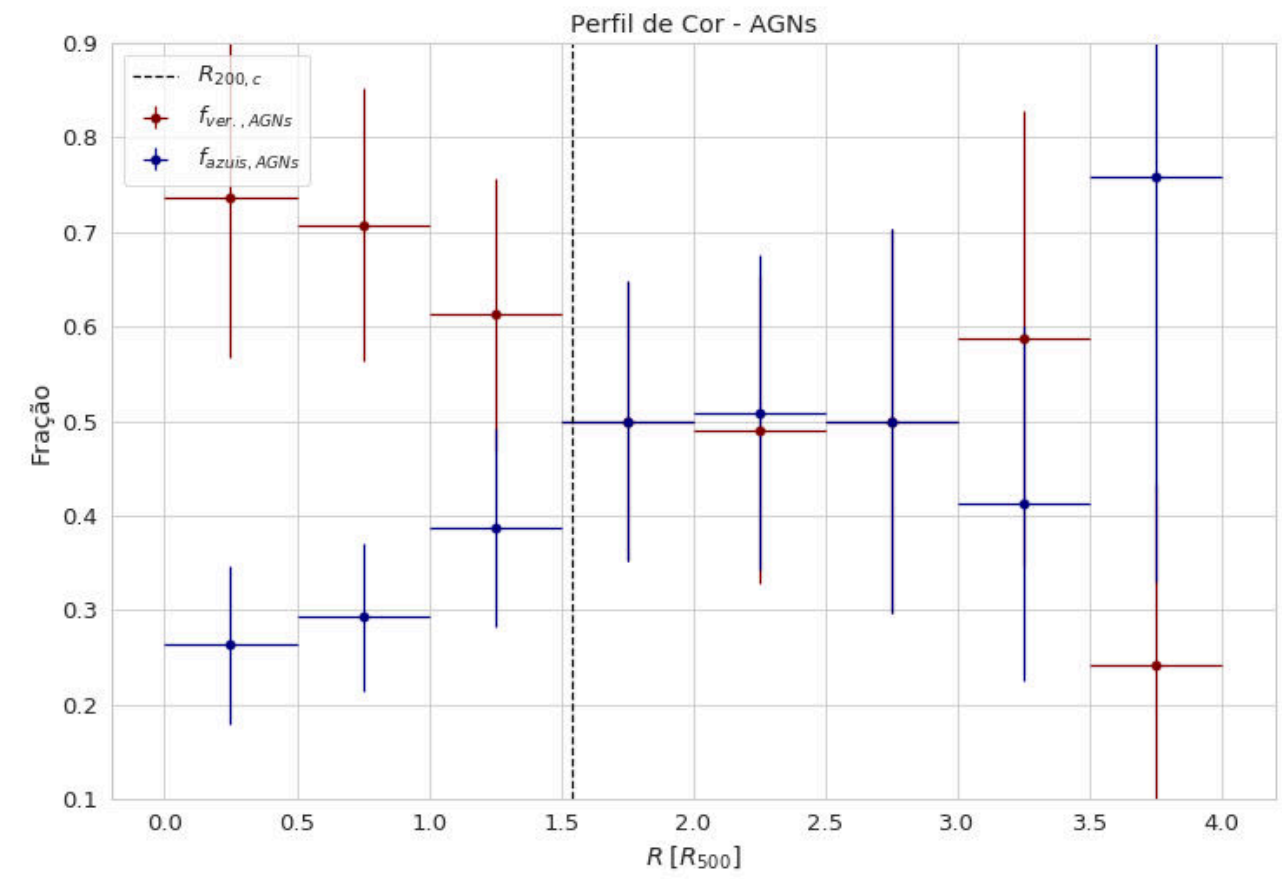

Figura 6.4: Perfil da fração de AGNs azuis e vermelhas em função do raio normalizado por $R_{500}$ para 468 objetos no intervalo $0.1<z<0.65$. As galáxias foram selecionadas nas cores $(g-r)$ e $(r-i)$ pelo método de contagens, com os mesmos cortes de magnitude da figura 6.5

No tocante a segregação morfológica das galáxias hospedeiras de AGNs, Aird et al. (2012) utilizando uma amostra de 242 AGNs selecionadas em raios-X no campo do COSMOS com redshift do PRIMUS1 mostrou que AGNs de campo não estão predominante em

\footnotetext{
${ }^{1}$ do inglês: $\boldsymbol{P R}$ ism $\boldsymbol{M U}$ Ulti-object $\boldsymbol{S}$ urvey
} 
galáxias vermelhas e nem massivas e sim na nuvem azul ou no vale verde. Sendo assim, considerando que existam mais AGNs azuis no campo, é necessário agora, apenas mostrar que existem mais AGNs vermelhas em aglomerados. Com efeito, observamos tal evidência, veja figura 6.4. A fração de AGNs vermelhas é cerca de $75 \%$ no centro e chega até valores iguais por volta de $R_{200, c}$ (definido na seção 4.1), se igualando as azuis na periferia do aglomerado com exceção em $4 R_{500}$ que chega a ter valores de campo. A fração de AGNs azuis é um pouco mais representativa que a fração de galáxias azuis, indicando um efeito de seleção ou um efeito real. Considerando que seja um efeito verdadeiro, temos que as AGNs possuem uma maior população de galáxias recém chegadas no aglomerado.

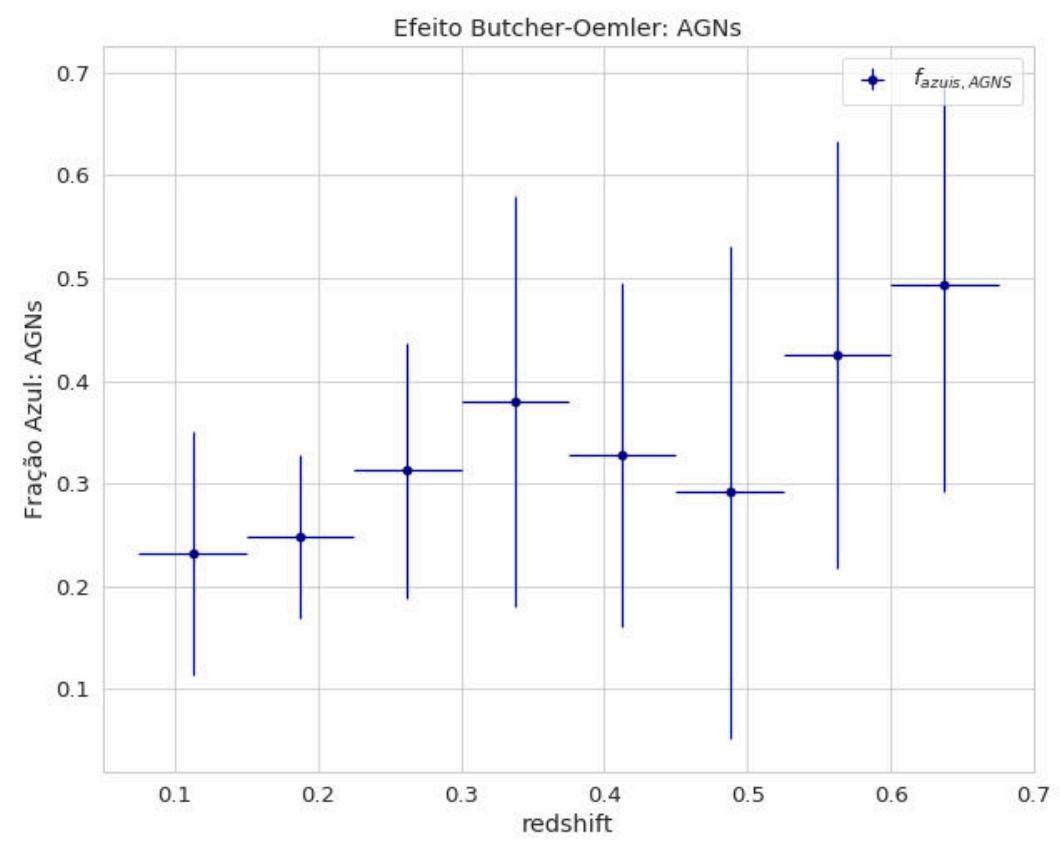

Figura 6.5: Evolução da fração de AGNs azuis dentro de $R_{200, c}$. São 262 AGNs com magnitudes $m<$ $m^{*}+3$. As galáxias azuis foram selecionadas nas cores $(g-r)$ e $(r-i)$. Os erros mostrados são Poissonianos.

Além da segregação morfológica se estender para as AGNs, outro resultado importante é que existe uma forte evolução da fração de AGNs azuis ao longo do redshift (figura 6.5), saindo de uma contribuição pouco significativa de $23 \%$ em $z=0.1$, tem um crescimento considerável e chega a ser $50 \%$ em $z=0.65$. Lembrando que os mesmos cortes de magnitudes aplicados na seção 5.2 foram também aplicados aqui. Notamos uma queda aparente por volta de $z \approx 0.4$, também encontrado na curva 5.3 indicando auto consistência do nosso método, porém a origem de tal efeito, nos faz pensar em algum viés da amostra do 
DES para galáxias vermelhas, é possível que esteja relacionada a um viés de redshift fotométricos (Palmese et al., 2016). Esse viés está relacionado com a falta de filtros próximos do ultravioleta. Além disso, esse comportamento pode ser devido à amostra de aglomerado ser selecionada por um algorítimo baseado na sequência vermelha.

A contaminação pela emissão do núcleo da AGN pode afetar as cores observadas da nossa amostra. Pierce et al. (2010) comparando a cor observada na região nuclear com a região externa de AGNs selecionadas em raios-X em $z \sim 1$ mostrou que em geral a cor integrada não é afetada, exceto em raros casos. Tais casos compõem menos que 10\% das galáxias com luminosidade em raios-X, $L_{X}>2 \times 10^{43} \mathrm{ergs}^{-1}$ e não obscurecidas. Então, acreditamos que a contaminação por AGN não afeta de maneira forte nosso estudo, com exceção dos mais objetos mais luminosos não obscurecidos da nossa amostra.

\subsection{Evolução}

Nesta seção apesentamos a fração de AGNs dentro do aglomerado ao longo do redshift. Discutimos uma possível evolução e a sua origem.

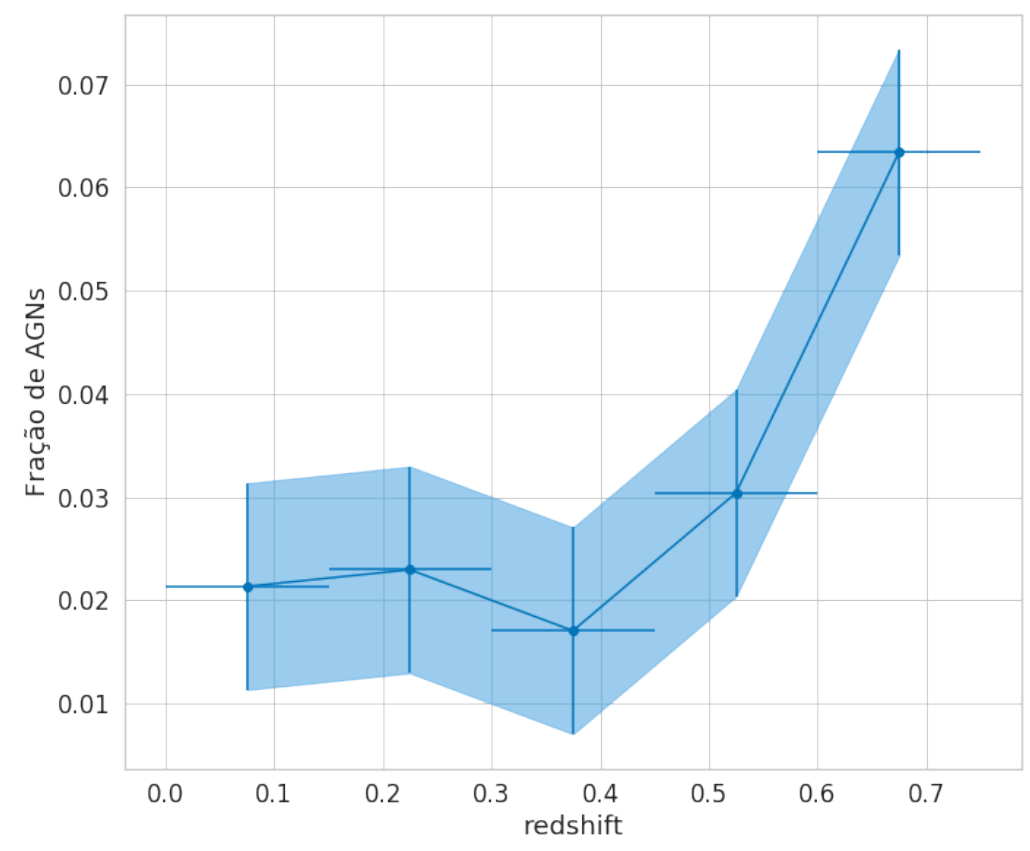

Figura 6.6: Fração de AGNs ao longo do redshift para toda galáxias hospedeiras dentro $4 R_{500}$ e com magnitudes $M_{r} \leq-19.8$. Os erros mostrados são Poissonianos. 
Na Figura 6.6 temos a $f_{\mathrm{AGNs}}$ dentro $4 R_{500}$ ao longo do redshift, é evidente uma forte evolução da $f_{\mathrm{AGNs}}$ depois de $z=0.45$. Porém, qual é a causa dessa evolução? Como vimos diferentes mecanismos atuam em diferentes regiões do aglomerado, sendo assim, é interessante estudarmos essa evolução com as regiões do aglomerado.

Na Figura 6.7 temos a mesma a relação anterior, porém, agora dividimos a relação em três regiões. E também dividimos entre AGNs com emissão hard (indicadoras de AGNs obscurecidas como vimos na seção 3.6) e soft. No gráfico da direita observamos que a evolução se dá na região externa do aglomerado. E também, tem como principal fonte, AGNs com emissão hard. Esse aumento na $f_{A G N s, H a r d}$ na periferia do aglomerado indica um cenário de queda de galáxias. Mais ainda, as AGNs hard são predominante em relações às com emissão soft. Assumindo que todas as AGNs com emissão hard são obscurecidas, os nossos resultados indicam uma evolução na fração de AGNs obscurecidas.

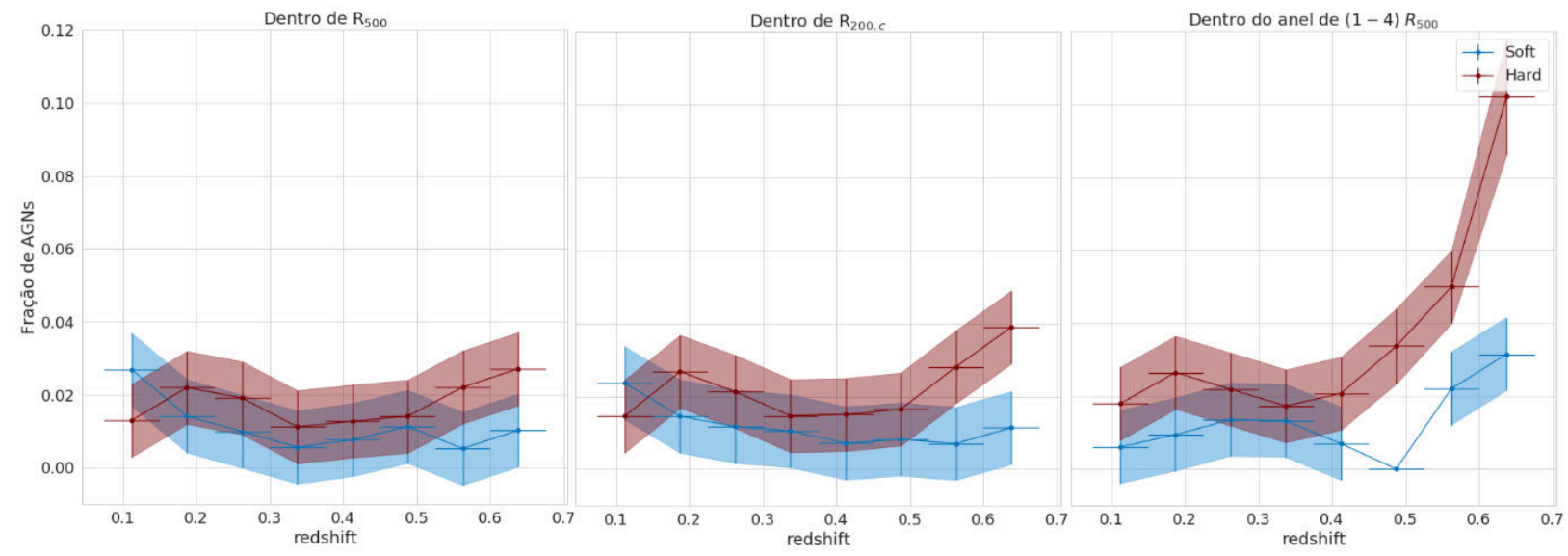

Figura 6.7: Fração de AGNs ao longo do redshift dividas em 3 regiões do aglomerado, $R_{500}, R_{200, c}$ e $(1-4) R_{500}$. Temos as fontes pontuais em raios-X: soft e hard. As fontes hard(soft) são indicadores de AGNs (não)obscurecidas. Notamos que a evolução está associada com as AGNs mais obscurecidas e nas regiões periféricas do aglomerado.

AGNs que foram ativadas por fusão de galáxias tem 5 vezes mais chance de serem obscurecidas do que galáxias ativas que não possuem sinais de fusão (Weston et al., 2017). Sendo assim, a evolução com redshift indica um cenário de acreção nas regiões mais externas do aglomerado e o mecanismo predominante pela atividade nuclear nessas regiões é fusão de galáxias.

Além disso, o pre-processamento de grupos em aglomerados podem explicar essa aumento da fração de AGNs com o aumento do redshift. Vijayaraghavan e Ricker (2013) mostrou que pelo menos um processo de fusão acontece entre as galáxias de um grupo 
caindo em um aglomerado antes desse sistema atingir sua menor distância do centro do aglomerado.

\subsection{O Papel Do Estado Dinâmico}

Nesta seções discutimos possíveis correlações da $f_{\mathrm{AGNs}}$ com as propriedades do ICM, massa e estado dinâmico.

É conhecido que fusão de galáxias pode ser um mecanismo eficiente em ativar núcleos ativos. Como a taxa de colisões depende inversamente da dispersão de velocidades ao cubo (Mamon, 1992), quanto maior a massa do aglomerado menor a possibilidade de tal evento ocorrer. Em regiões mais externas, é mais provável haver uma colisão. E no centro do aglomerados, encontros rápidos, conhecidos como assédio galáctico, podem causar uma mudança morfológica nas galáxias. Em específico, galáxias espirais tipo Sc e Sd tem seu disco destruído e seu bojo aumentado devido a esse fenômeno (Moore et al., 1998). Além disso, esses efeitos podem ativar um fase nuclear nas galáxias desde que o gás sofra um torque na direção central do sistema.

Nesse sentido Ehlert et al. (2015) defende um cenário de AGNs engatilhadas por fusões, sua tese é baseado em um ajuste de relação de escala, $N_{\mathrm{AGNs}} \propto M_{200}^{-1.2_{-0.8}^{+0.3}}$, que tem uma semelhança com taxa de fusões assumindo a massa do virial, $\propto M_{200}^{-1}$. Mas, tal afirmação tem problemas, não há nenhum cálculo teórico e simulação que comprove tal correlação. Mais ainda, recentemente, Deger et al. (2018) mostraram que a fração de galáxias em fusões ou com interações por forças de maré $\left(f_{\text {TIM }}\right)$ não tem correlação com a dispersão de velocidade, e apesar da tentativa, não encontraram fortes evidências de que $f_{T I M}$ tem valores mais altos em aglomerados e grupos do que em regiões de campo, com exceção de regiões mais externas dos aglomerados, $0.5 R_{200}$. Por outro lado, a pressão de arraste é mais eficiente em aglomerados de maior massa. Sendo assim, se faz necessário uma simulação ou outras correlações a fim de distinguir as causas dessa supressão de AGNs com a massa do aglomerado.

Feitas essas considerações, também encontramos uma supressão da fração de AGNs com a massa do aglomerado, conforme vemos na Figura 6.8. No entanto, isso não é o suficiente para afirmar que seja devido a interações colisionais. Também não excluímos a hipótese de ser devido a pressão de arraste. 


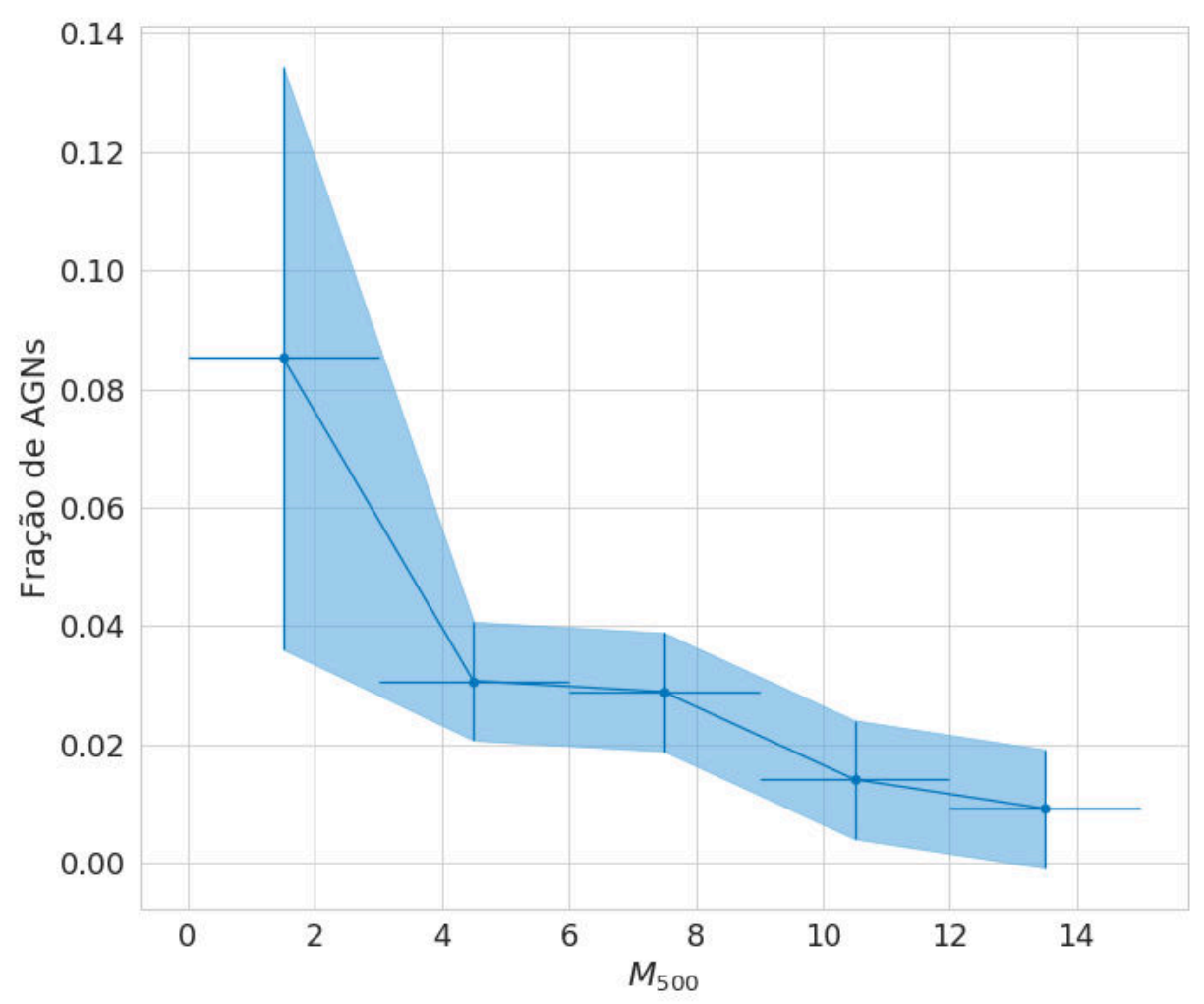

Figura 6.8: Dependência da atividade nuclear com a massa do aglomerado. Fração de AGNs dentro de $R_{500}$ em função de $M_{500}$.

De fato, simulações hidrodinâmicas de Ruggiero e Lima Neto (2017) mostraram que uma galáxias tipo Via Láctea perde todo seu gás por pressão de arraste ao chegar no centro de um sistema cool-core (daqui em diante, CC), enquanto em um sistema não CC a perda total nunca acontece na primeira passagem. Em particular, a fração de gás remanescente em sistemas não CC depende da massa do aglomerado. Tomando como compasso a nossa galáxia para todas o conjunto das galáxias azuis, tiramos duas conclusões desse estudo, a pressão de arraste depende da massa do aglomerado e é mais eficiente em aglomerados CC.

Motivados por essas afirmações, investiguemos aglomerados CC, na Figura 6.9 temos a $f_{\mathrm{AGNs}}$ dentro de $R_{500}$ em função de $c_{\mathrm{SB}}$ (indicador de sistemas cool-core). Vemos que a $c_{\mathrm{SB}}$ tem uma anti-correlação com a $f_{\mathrm{AGNs}}$. Ou seja, quanto mais intenso é o cooling-flow menor é a $f_{\mathrm{AGNs}}$ dentro de $R_{500}$. Temos que esse resultado é único, não encontramos na literatura nenhum estudo que investiga essa correlação.

Sendo assim levantamos três cenários: (i) a pressão de arraste é mais eficiente em sistemas CC, as galáxias tipo disco perdem seu gás antes de chegar ao centro do aglomerado, 


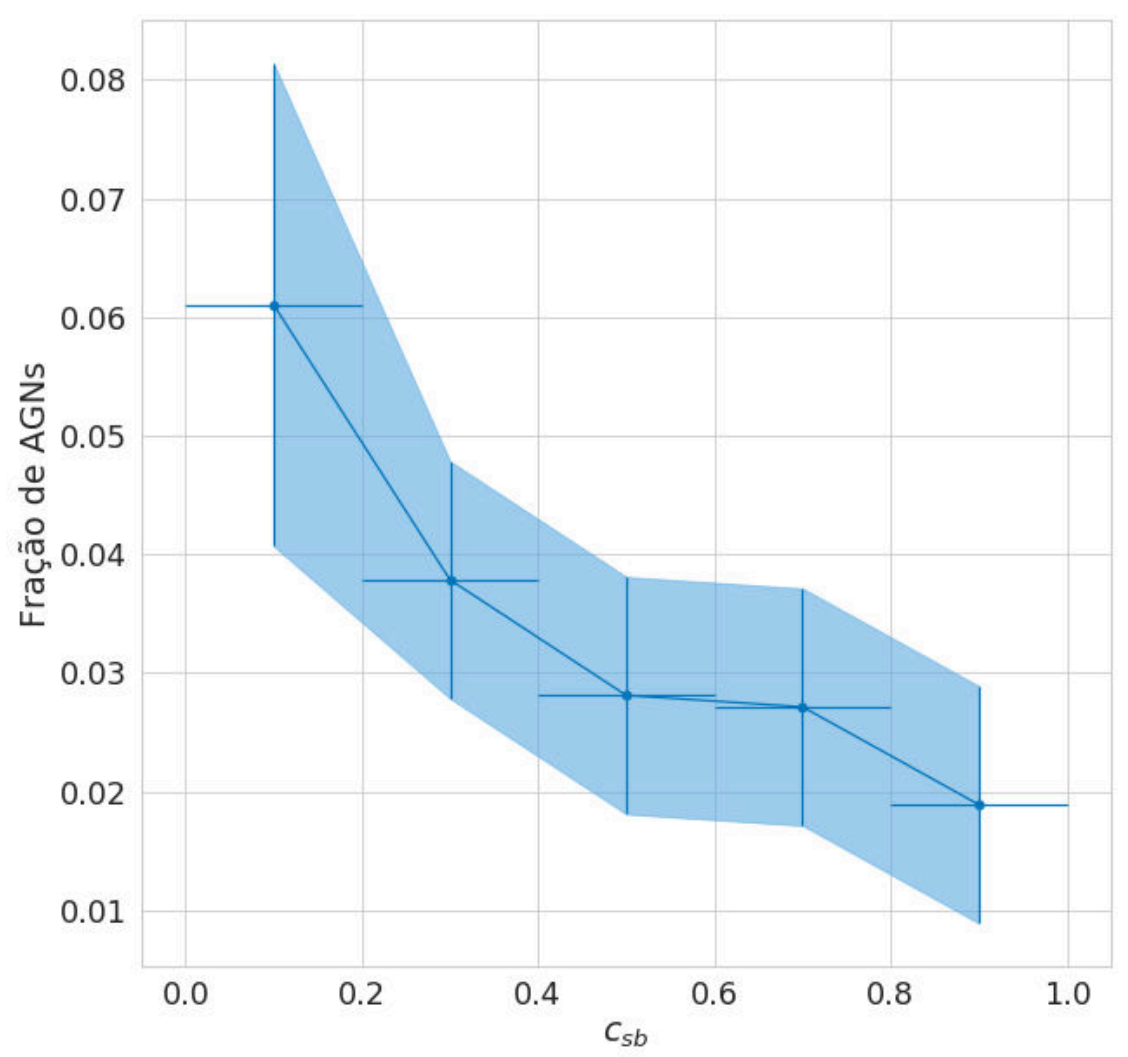

Figura 6.9: Fração de AGNs dentro de $R_{500}$ em função da concentração, $c_{S B}$ - indicador de sistemas cool-core.

devido ao esgotamento do reservatório de gás, temos uma ocorrência menor de AGNs; (ii) aglomerados CC são mais dinamicamente relaxados, não possuem um histórico recente de acreção, as galáxias já perderam todo seu gás frio, consequentemente, possuem uma fração menor de AGNs; (iii) o cooling-flow é contrabalanceado não apenas pela AGN central, mas também, por toda população de AGNs do aglomerado.

O primeiro e segundo cenários assume que a AGNs são uma população de galáxias em queda no aglomerado. Em particular no primeiro cenário temos que a razão pelo esgotamento do reservatório de gás é pressão de arraste, e que este mecanismo é mais eficiente em aglomerados CC, explicando assim a anti-correlação observada. Além disso, esse cenário é corroborado pelas evidências desse estudo, isto é, a anti-correlação com a massa do aglomerado, a supressão de AGNs na região central do aglomerado e o efeito Butcher-Oemler das AGNs.

No segundo cenário temos a anti-correlação observada está ligada ao aglomerado ser dinamicamente relaxado e não ter acretado galáxias recentemente. Nesse sentido, temos 
que deve existir uma correlação da $f_{\mathrm{AGNs}}$ com o estado dinâmico.

Em relação ao terceiro cenário, apesar do feedback de AGNs da galáxia central ser considerado a solução mais viáveis para a solução do problema do cooling-flow (McNamara e Nulsen, 2007). Esse anti-correlação levanta a possibilidade que existe uma possibilidade de que a energia líquida produzida por AGNs em todo aglomerado pode contrabalancear o cooling-flow. Essa conexão pode existir pois, os modelos de Kaiser (1991); Evrard e Henry (1991) explicam que se houver fontes de energia injetadas no sistema em $z \gtrsim 6$ é possível explicar a ausência de sistemas CC como predito pelo modelo clássico de cooling-flow. Logo, seria interessante realizar simulação cosmológicas hidrodinâmicas a fim de estudar esse cenário.

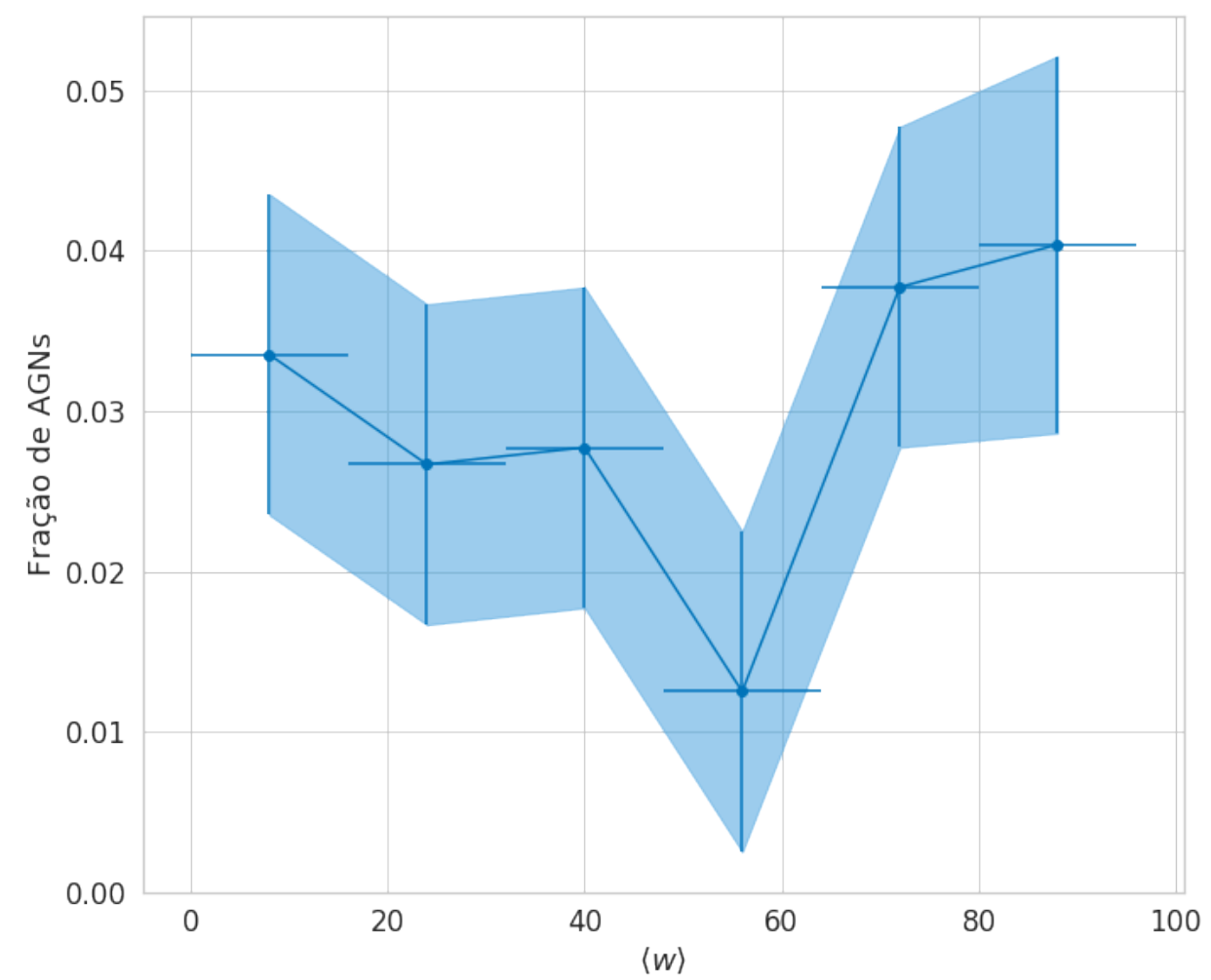

Figura 6.10: Fração de AGNs em função do desvio do centro $\langle w\rangle$ - indicador de pertubação do estado dinâmico.

Seguindo a lógica levanta pelo segundo cenário, em aglomerados dinamicamente ativos se espera haver uma maior fração de AGNs. Na Figura 6.10 temos a $f_{\text {AGNs }}$ em função do desvio do centro, $\langle w\rangle$, e não observamos essa correlação proposta nesse cenário. Podemos apenas dizer que em sistemas altamente perturbados temos uma fração de AGNs maior, porém, nada significativo. 


\subsection{Efeitos de Ambiente em AGNs}

Em nosso trabalho, discutimos dois possíveis mecanismos para explicar a dependência do ambiente nas AGNs em aglomerados, encontros colisionais e interações do meio intraaglomerado com as galáxias (pressão de arraste).

Na literatura é amplamente discutido os mecanismos responsáveis por disparar a atividade nuclear, no entanto, esse estudo aponta que a questão deve ser colocada colocada sob o ponto de vista do reservatório de gás frio. Esse reservatório não é só essencial por manter a atividade nuclear, mas também a taxa de formação estelar.

De fato, mostramos que a segregação cor(morfologia) e o efeito Butcher Oemler se estende para as AGNs (veja a seção 6.2). Indicando assim que os efeitos ambientais evolutivos de galáxias azuis e AGNs estão intrinsecamente ligados pelo mesmo fenômeno. Nesse caso, o esgotamento do reservatório de gás. E o mecanismo responsável não apenas pela diminuição de galáxias azuis, mas também para as AGNs é a pressão de arraste.

Esse efeito é importante sobretudo nas regiões centrais do aglomerado, onde temos uma supressão na fração de AGNs (veja o Gráfico 6.1). Além disso, a pressão de arraste também pode ser responsável por disparar a atividade nuclear e nossos resultados estão de acordo com as simulações hidrodinâmicas que estudaram esse efeito (Marshall et al., 2018).

Pela primeira vez mostramos que existe uma diminuição gradual da fração de AGNs dentro de $R_{500}$ com a intensidade do cooling-flow (veja o Gráfico 6.9). Resultado em acordo com Ruggiero e Lima Neto (2017) que mostrou que a queda de uma galáxia tipo Via Láctea perde todo seu gás por pressão de arraste ao atingir o centro de um aglomerado CC enquanto que para um aglomerado não CC permanece uma certa quantidade da fração de gás inicial.

Além disso, também encontramos que existe uma diminuição gradual da fração de AGNs dentro de $R_{500}$ com a massa do aglomerado (veja o Gráfico 6.8). Ehlert et al. (2015) observou a mesma anti-correlação, porém, em seu trabalho defendem que esse resultado é devido a interações galácticas. No entanto, esse correlação com a massa também pode ser devido a pressão de arraste. Em particular, na simulação de Ruggiero e Lima Neto (2017) a fração de gás remanescente da galáxia em um aglomerado de massa $10^{15} M_{\odot}$ foi entre $16 \%-28 \%$ e para um aglomerado de massa de massa $10^{14} M_{\odot}$ foi entre $43 \%-54 \%$. Ou seja, a pressão de arraste é mais eficiente em aglomerados de maior massa. 
Temos que essas observações favorece fortemente para nosso tese que a pressão de arraste é o mecanismo predominante dentro das regiões centrais do aglomerado, pois, se o mecanismo predominante para a atividade nuclear fosse interações galácticas não deveríamos encontrar diferenças na população de AGNs em sistemas CC.

Se por um lado mostramos que a pressão de arraste é o mecanismo predominante nas regiões centrais. Por outro encontramos evidências que fusões de galáxias devem ter um papel importante nas regiões mais externas. Observamos uma forte evolução com o redshift da fração de AGNs com emissão hard na zona de infall. Assumindo que AGNs com emissão hard são obscurecidas e que esse obscurecimento, apontamos para um cenário que a fusão de galáxias deve ter sido predominante nas regiões externas em uma época de próxima à formação dos aglomerados.

Esse observação está de acordo com o cenário hierárquico e a teoria de pre processamento de grupos de galáxias em aglomerados. E segundo a teoria de pre processamento as galáxias do aglomerado tem sua propriedades devido a acresção de grupos de galáxias e filamentos. De fato, galáxias em grupos de galáxias caindo em aglomerados tem sua taxa de fusões amplificadas (Vijayaraghavan e Ricker, 2013).

Em suma, a pressão de arraste é o mecanismo predominante nas regiões centrais do aglomerados pela fração de AGNs observada. Nas regiões externas, a fusão de galáxias pode ter sido predominante em disparar a atividade nuclear durante a fase de crescimento dos aglomerados. Em particular, essas fusões pode ter sido no ambiente de grupos pre processados pelo aglomerado. Na Figura 6.11 ilustramos o cenário geral proposto nesse trabalho. 


\section{Grupo Galáxias}
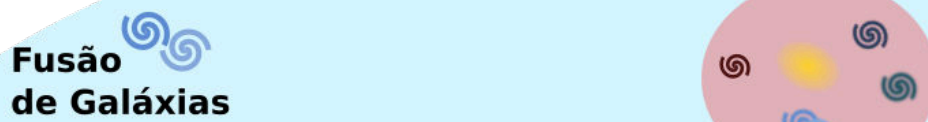

de Galáxias
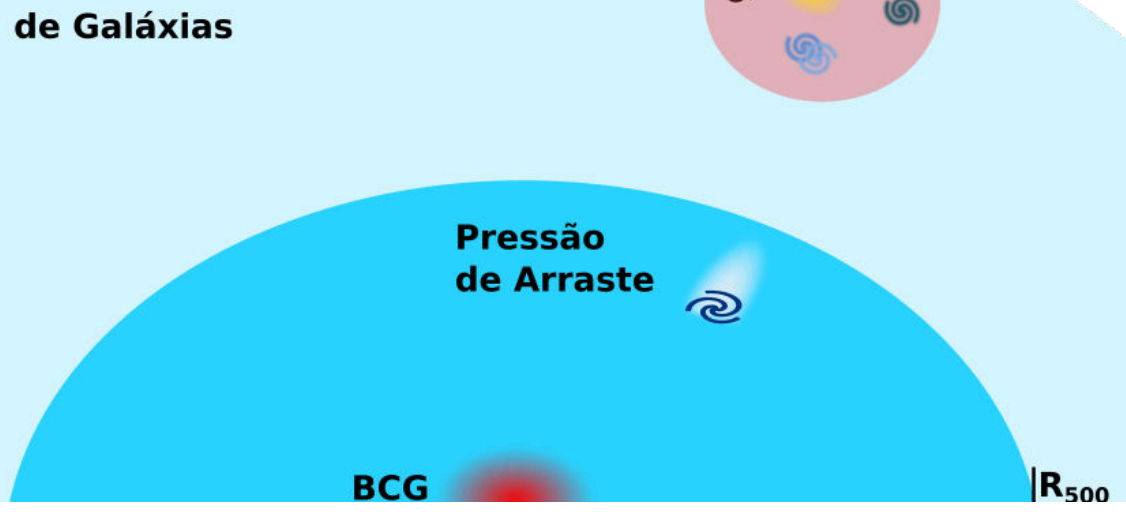

Figura 6.11: Ilustração do cenário geral proposto nesse trabalho. 


\section{Conclusões}

O principal objetivo deste trabalho é investigar a relação dos efeitos ambientais em aglomerados de galáxias com a atividade de galáxias de núcleo ativo (AGN). Através de evidências observacionais, estudamos a demografia das AGNs e sua evolução ao longo do redshift. Também, utilizamos sua cor em raios-X, hardness-ratio, para investigar o obscurecimento das galáxias hospedeiras. E assim, estudamos a correlação dessas propriedades com a massa total, temperatura e estado dinâmico do aglomerado. Similarmente, também revisitamos relações ambientais entre o aglomerado e a sua população galáctica como um todo.

Nosso estudo explorou um intervalo de redshift largo, de 0.1 até 0.65 . Utilizou a força da astronomia multi-bandas, raios-X e ótico. A nossa amostra no óptico contou com dois dos maiores levantamentos fotométricos atuais, DES e SDSS. Um catálogo de aglomerados que já tinha suas propriedades físicas estimadas (Maughan et al., 2012) foi utilizado para os objetos no campo do SDSS. De maneira consistente, aplicamos a mesma metodologia na estimativa das propriedades físicas dos aglomerados no campo do DES.

Do ponto de vista de raios-X, estimamos não só as propriedades físicas como massa e temperatura, como também caracterizamos o estado dinâmico do ICM, utilizando os indicadores $c_{S B}$ e $\langle w\rangle$. Criamos e disponibilizamos Xpip€ $\rrbracket^{1}$, um código que automatiza as análises em raios-X realizadas neste trabalho. Além disso, criamos nosso catálogo de AGNs selecionadas em raios-X, com aproximadamente 1000 objetos em 96 aglomerados.

A população galáctica em ambientes de aglomerados de galáxias apresenta propriedades distintas em relação às galáxias de campo. Esse ambiente de alta densidade separa os tipos morfológicos. As espirais que são maioria no campo, são a minoria em aglomerados

\footnotetext{
${ }^{1}$ Github: https://github.com/estevesjh/Xpipe
} 
de galáxias, apenas $20 \%$ das galáxias na região central. Esse fenômeno é conhecido como segregação morfológica (Dressler, 1980). Em nosso trabalho, utilizando cores como indicadores morfológicos, encontramos essa relação ao longo do raio do aglomerado com uma remarcável concordância dentro de $5 \%$ de diferença quando comparada com resultados de Whitmore et al. (1993). Não encontramos evidências fortes para correlação dessa relação com as propriedades do meio intra-aglomerado.

Butcher e Oemler 1978, 1984) observaram um aumento substancial da fração de galáxias azuis $\left(f_{b}\right)$ até $z \approx 0.4$, indicando pela primeira vez um fator evolutivo das galáxias no ambiente denso de aglomerados. Porém, desde o início esse efeito foi debatido, devido estar sujeito a um série de possíveis efeitos de seleção (Andreon e Quintana, 2006). Com efeito, observamos o efeito Butcher-Oemler até $z \approx 0.4$, porém, após esse intervalo, o efeito atinge uma espécie de plateau e volta a crescer em $z>0.6$.

Em particular, para os objetos que apresentaram uma fração de galáxias azuis não crescente, não excluímos a hipótese de anti-correlação de $f_{b}$ com as propriedades físicas do aglomerado, como massa, temperatura e $Y_{X}$ - indicador de pressão. Caso essa correlação exista, propomos um cenário em que a pressão de arraste é responsável pela diminuição de galáxias azuis, pois, quanto maior a pressão do meio intra-aglomerado (ICM) mais efetivo esse mecanismo. Também procuramos alguma correlação com o estado dinâmico, porém encontramos apenas uma leve tendência.

Por outro lado, vieses de seleção podem ser a causa desse não crescimento de $f_{b}$ em redshift intermediário. Acreditamos que o redshift fotométrico pode ser responsável, pois galáxias azuis geralmente são menos luminosas e possuem redshift fotométricos mais complexos de serem estimados. Em especifico, Palmese et al. (2016) notou um viés dos redshifts fotométricos do DES quando comparado com dados do COSMOS, em torno de $z \approx 0.4$.

Sobre a evolução de AGNs em aglomerados, analisamos a demografia desses objetos nesse meio. Constatamos a conhecida dependência demográfica dessas galáxias com o aglomerado. Conforme vimos na figura 6.3, existe uma fração menor de AGNs no centro e maior na região por volta do raio do virial. Discutimos os resultados anteriores que contrariam tal observação, mostramos que quando se utiliza apenas o número de AGNs encontradas em raios-X com contrapartida ótica há uma sobredensidade central, no entanto, essa medida é enganosa, pois não leva em conta a população de galáxias como um todo. 
Investigamos o mecanismo responsável pela dependência das AGNs com o ambiente de aglomerados. Mostramos que as AGNs, assim como as galáxias azuis, possuem um efeito Butcher-Oemler significativo. Em $z=0.1$ a fração de galáxias azuis hospedeiras de AGNs é apenas um quinto e em $z=0.65$ passam a ser mais da metade da população de AGNs no aglomerado. Também mostramos que a segregação cor (morfológica) se estende para as galáxias de núcleo ativo. Corroborando assim para um cenário em que a pressão de arraste é responsável, não só pela diminuição das galáxias formadoras de estrelas, mas também para as galáxias de núcleo ativo, evidenciando ainda mais essa conexão entre a formação estelar e a atividade nuclear.

Ainda sobre os efeitos ambientais, a pressão de arraste sofrida pelas galáxias ao cair no aglomerado pode não apenas arrancar o gás, mas também pode induzir a atividade nuclear em um certo regime de pressão. Simulações hidrodinâmicas realizadas por Marshall et al. (2018), investigaram esse efeito e mostraram que a curva da $f_{\mathrm{AGNs}}$ ao longo do raio do aglomerado teria um pico no raio do virial. Nossos resultados corroboram para tal cenário.

Além disso, fusões entre galáxias pode ser a causa da atividade nuclear na periferia dos aglomerados observados nesse trabalho. Encontramos uma forte evolução com o redshift de AGNs na região $(1-4) \times R_{500}$ do aglomerado. Mais ainda, essa evolução é causada por galáxias hospedeiras que são obscurecidas. Interpretamos essa forte evolução da $f_{\mathrm{AGNs}}$ obscurecidas como sendo uma consequência de que a queda de galáxias foi mais predominante no passado, quando os aglomerados tinham uma maior taxa de acresção de galáxias do campo e grupos de galáxias. O mecanismo dominante para a atividade das AGNs era por fusões.

Nesse sentido Ehlert et al. (2015) defende um cenário de AGNs engatilhadas por fusões, sua tese é baseado em um ajuste de relação de escala, $N_{A G N s} \propto M_{200}^{-1.2_{-0.8}^{+0.3}}$, que tem uma semelhança com taxa de fusões assumindo a massa do virial, $\propto M_{200}^{-1}$. Também observamos essa tendência, veja figura 6.8. No entanto, a pressão de arraste também é mais eficiente em aglomerados de maior massa, logo, não podemos distinguir o mecanismo responsável por essa observação, é necessário outros resultados para retirarmos conclusões.

Por fim, encontramos uma anti-correlação da intensidade do cooling-flow com a fração de AGNs. Esse resultado suscita três cenários possíveis.

1. A pressão de arraste é mais eficiente em sistemas CC, já que as galáxias tipo disco perdem seu gás antes de chegar ao centro do aglomerado. Sendo assim, devido ao 
esgotamento do reservatório de gás frio, temos uma ocorrência menor de AGNs.

2. Aglomerados CC são mais dinamicamente relaxados e não possuem um histórico recente de acreção. Consequentemente, temos uma fração menor de AGNs, pois, as galáxias nesses ambientes já perderam quase todo seu gás frio.

3. O cooling-flow é contrabalanceado não apenas pela AGN central, mas também, por toda população de AGNs do aglomerado.

O primeiro cenário, está de acordo com nossas conclusões sobre a evolução de galáxias e de AGNs em aglomerados. Além do mais, essa anti-correlação da $f_{\text {AGNs }}$ com a intensidade do CC é um resultado único em favor da nossa tese que a pressão de arraste é o mecanismo predominante dentro das regiões centrais do aglomerado, pois, se o mecanismo predominante para a atividade nuclear fosse interações galácticas não deveríamos encontrar diferenças na população de AGNs em sistemas CC.

Em relação ao segundo cenário, devemos espera haver uma maior fração de AGNs em aglomerados dinamicamente ativos. Entretanto, não encontramos uma correlação da $f_{\mathrm{AGNs}}$ com o desvio do centro, $\langle w\rangle$ (indicado de sistemas perturbados). Podemos apenas dizer que em sistemas altamente perturbados temos uma fração de AGNs maior, porém, nada significativo.

Os modelos de Kaiser (1991); Evrard e Henry (1991) indicam que o terceiro cenário é possível. Mas, nesse modelo as fontes de energia injetam energia no sistema em um redshift mais alto do que deste trabalho, portanto, seria interessante investigar esse cenário em simulações hidrodinâmicas.

\subsection{Perspectivas}

Esperamos expandir a nossa amostra até redshift $z \approx 1$ com os dados do DES Y3. Com esse aumento substancial no volume de dados esperamos investigar em alto redshift os efeitos de ambiente em AGNs e na população de galáxias do aglomerado como um todo. Além disso, também esperamos explorar essa metodologia com dados futuros do DESI (Collaboration, 2019) e do J-PAS (Benitez et al., 2014).

Do ponto de vista técnico, esperamos melhorar as estimativas de redshift fotométricos das AGNs com os dados em infravermelho médio de todo céu do levantamento unWISE 
2. Com adição de mais duas bandas nesse comprimento é possível ter uma melhora significativa, principalmente para as galáxias azuis. Além disso, pensamos na possibilidade de adicionar as bandas em raios-X ao ajuste redshift fotométrico.

Com o aprimoramento dos redshifts fotométricos, poderemos investigar o possível viés por volta de $z \approx 0.4$. Também, seria interessante verificar se esse viés é devido ao catálogo do redMaPPer. Com os aglomerados selecionados pelo efeito Sunyaev-Zeldovich, podemos aplicar nossa metodologia aqui desenvolvida e comparar a evolução de $f_{b}$.

No futuro, esperamos explorar as implicações dos três cenários propostos para a anti correlação da intensidade do cool-core com a fração de AGNs. Simulações hidrodinâmicas cosmológicas seria uma boa forma de discriminar a importância desses cenários. Nesse sentido, podemos estabelecer colaborações com grupos de simulação. Em relação ao segundo cenário, pretendemos explorar a correlação da taxa de formação estelar do aglomerado com a sua população de galáxias. Graças à nossa colaboração com o grupo da Prof. Soares-Santos na "Brandeis University"já possuímos as ferramentas necessárias para esse trabalho.

O DESI por ser um levantamento espectroscópico e o J-PAS com as suas 56 bandas que terá uma precisão na estimativa dos photo-z que é comparável ao redshift obtido por espectros de baixa qualidade promoverá uma melhoria significativa na seleção de galáxias membros e de AGNs do aglomerado.

\footnotetext{
${ }^{2}$ home page: http://unwise.me
} 


\section{Referências Bibliográficas}

Abell G. O., , ApJS, 1958, vol. 3, p. 211

Aird J., Coil A. L., et al., , ApJ, 2012, vol. 746, p. 90

Alexander D. M., Bauer F. E., et al., , ApJ, 2005, vol. 632, p. 736

Andrade-Santos F., Análise de Subestruturas em Raios-X em Aglomerados de Galáxias, Universidade de São Paulo - IAG/USP, 2008, Dissertação de Mestrado, 94 p.

Andrade-Santos F., Jones C., et al.,, ApJ, 2017, vol. 843, p. 76

Andreon S., Lobo C., Iovino A., , MNRAS, 2004, vol. 349, p. 889

Andreon S., Quintana H. o., , MNRAS, 2006, vol. 365, p. 915

Arnaud M., Pointecouteau E., Pratt G. W., , A\&A, 2005, vol. 441, p. 893

Bahcall N. A., Clusters and superclusters of galaxies. In Formation of Structure in the Universe , 1999, p. 135

Balogh M. L., Pearce F. R., et al., , MNRAS, 2001, vol. 326, p. 1228

Barnes J. E., , MNRAS, 2002, vol. 333, p. 481

Bastos T. C. M., Evolução de galáxias em ambientes de alta densidade, Instituto Nacional de Pesquisas Espaciais - INPE, 2017, Tese de Doutorado, 127 p.

Benítez N., , ApJ, 2000, vol. 536, p. 571

Benitez N., Dupke R., Collaboration J.-P., , arXiv e-prints, 2014, p. arXiv:1403.5237

Birkinshaw M., , Phys. Rep., 1999, vol. 310, p. 97

Boldt E., McDonald F. B., et al.,, Phys. Rev. Lett., 1966, vol. 17, p. 447

Bonamente M., Joy M. K., et al., , ApJ, 2006, vol. 647, p. 25 
Bower R. G., Benson A. J., et al., , MNRAS, 2006, vol. 370, p. 645

Butcher H., Oemler A. J., , ApJ, 1978, vol. 219, p. 18

Butcher H., Oemler A. J., , ApJ, 1984, vol. 285, p. 426

Collaboration D., , arXiv e-prints, 2019, p. arXiv:1907.10688

Deger S., Rudnick G., et al., , ApJ, 2018, vol. 869, p. 6

Dickey J. M., Lockman F. J., , ARA\&A, 1990, vol. 28, p. 215

Dressler A., , ApJ, 1980, vol. 236, p. 351

Dressler A., Oemler Augustus J., et al., , ApJ, 1997, vol. 490, p. 577

Dressler A., Thompson I. B., Shectman S. A., , ApJ, 1985, vol. 288, p. 481

Eastman J., Martini P., et al., , ApJ, 2007, vol. 664, p. L9

Ehlert S., Allen S. W., et al.,, MNRAS, 2015, vol. 446, p. 2709

Ellis R. S., Smail I., et al., , ApJ, 1997, vol. 483, p. 582

Evans I. N., Primini F. A., et al., , ApJS, 2010, vol. 189, p. 37

Evrard A. E., Henry J. P., , ApJ, 1991, vol. 383, p. 95

Fabbiano G., , ARA\&A, 2006, vol. 44, p. 323

Fabian A. C., , ARA\&A, 1994, vol. 32, p. 277

Felten J. E., Gould R. J., et al.,, ApJ, 1966, vol. 146, p. 955

Ferrarese L., Merritt D., , ApJ, 2000, vol. 539, p. L9

Ferreras I., Charlot S., Silk J., , ApJ, 1999, vol. 521, p. 81

Flaugher B., Diehl H. T., The DES Collaboration, AJ, 2015, vol. 150, p. 150

Forman W., Jones C., et al., , ApJ, 2007, vol. 665, p. 1057

Freeman P., Doe S., Siemiginowska A., . In Astronomical Data Analysis, vol. 4477 of Proc. SPIE, 2001, p. 76

Fruscione A., McDowell J. C., et al., , vol. 6270 of SPIE, 2006, p. 62701V

Gebhardt K., Bender R., et al.,, ApJ, 2000, vol. 539, p. L13 
Gilmour R., Gray M. E., et al., , MNRAS, 2007, vol. 380, p. 1467

Gisler G. R., , MNRAS, 1978, vol. 183, p. 633

Gladders M. D., López-Cruz O., Yee H. K. C., Kodama T., , ApJ, 1998, vol. 501, p. 571

Gladders M. D., Yee H. K. C., , AJ, 2000, vol. 120, p. 2148

Gómez P. L., Nichol R. C., et al., , ApJ, 2003, vol. 584, p. 210

Gordon Y. A., Pimbblet K. A., et al.,, MNRAS, 2018, vol. 12, p. 1

Goto T., Yamauchi C., et al., , MNRAS, 2003, vol. 346, p. 601

Gursky H., Kellogg E., et al.,, ApJ, 1971, vol. 167, p. L81

Haggard D., Green P. J., et al., , ApJ, 2010, vol. 723, p. 1447

Hao J., Koester B. P., et al.,, ApJ, 2009, vol. 702, p. 745

Hennig C., Mohr J. J., others. , MNRAS, 2017, vol. 467, p. 4015

Hickox R. C., Alexander D. M., , ARA\&A, 2018, vol. 56, p. 625

Hickox R. C., Jones C., et al., , ApJ, 2009, vol. 696, p. 891

Holland J. G., Böhringer H., et al., , MNRAS, 2015, vol. 448, p. 2644

Hollowood D. L., Jeltema T., et al.,, arXiv e-prints, 2018

Hopkins P. F., Hernquist L., et al., , ApJS, 2008, vol. 175, p. 356

Hopkins P. F., Somerville R. S., et al., , MNRAS, 2009, vol. 397, p. 802

Hubble E., Humason M. L., , ApJ, 1931, vol. 74, p. 43

Hubble E. P., , ApJ, 1926, vol. 64, p. 321

Hubble E. P., , 1936

Kaiser N., , ApJ, 1991, vol. 383, p. 104

Kauffmann G., White S. D. M., et al., , MNRAS, 2004, vol. 353, p. 713

Kormendy J., Richstone D., , ARA\&A, 1995, vol. 33, p. 581

Koulouridis E., Bartalucci I., , A\&A, 2019, vol. 623, p. L10

Koulouridis E., Plionis M., et al., , A\&A, 2014, vol. 567, p. A83 
Kravtsov A. V., Vikhlinin A., Nagai D., , ApJ, 2006, vol. 650, p. 128

Lacey C., Cole S., , MNRAS, 1993, vol. 262, p. 627

Laganá T. F., Martinet N., et al., , A\&A, 2013, vol. 555, p. A66

Lehmer B. D., Basu-Zych A. R., et al., , ApJ, 2016, vol. 825, p. 7

Lewis I., Balogh M., et al., , MNRAS, 2002, vol. 334, p. 673

Lima Neto G. B., , 2009 Tese de Livre Docência

Lima Neto G. B., , 2018 Notas de Aula em Astronomia Extragaláctica

Loh Y.-S., Ellingson E., et al., , ApJ, 2008, vol. 680, p. 214

Lopes P. A., Ribeiro A. L., Rembold S. B., , MNRAS, 2017, vol. 472, p. 409

Lotz J. M., Jonsson P., et al., , ApJ, 2011, vol. 742, p. 103

Mamon G. A., , ApJ, 1992, vol. 401, p. L3

Man Z., Peng Y., et al., , MNRAS, 2019, vol. 488, p. 89

Margoniner V. E., de Carvalho R. R., , AJ, 2000, vol. 119, p. 1562

Marshall M. A., Shabala S. S., et al.,, MNRAS, 2018, vol. 474, p. 3615

Martini P., Sivakoff G. R., Mulchaey J. S., , ApJ, 2009, vol. 701, p. 66

Maughan B. J., Giles P. A., et al.,, MNRAS, 2012, vol. 421, p. 1583

Maughan B. J., Jones C., et al.,, ApJS, 2008, vol. 174, p. 117

McDonald M., McNamara B. R., et al.,, arXiv e-prints, 2019

McNamara B. R., Nulsen P. E. J., , ARA\&A, 2007, vol. 45, p. 117

Mei S., Holden B. P., et al., , ApJ, 2009, vol. 690, p. 42

Moore B., Katz N., et al., , Nature, 1996, vol. 379, p. 613

Moore B., Lake G., Katz N., , ApJ, 1998, vol. 495, p. 139

Moré J., , 1978 in Watson G., ed., Lecture Notes in Mathematics, Vol. 630, Numerical Analysis. Springer Berlin Heidelberg pp 105-116

Nishizawa A. J., Oguri M., et al., , PASJ, 2018, vol. 70, p. S24 
Padmanabhan T., Structure Formation in the Universe, 1993, 499

Palmese A., Annis J., et al.,, arXiv e-prints, 2019

Palmese A., Lahav O., et al., , MNRAS, 2016, vol. 463, p. 1486

Peng Y., Maiolino R., Cochrane R., , Nature, 2015, vol. 521, p. 192

Pereira M. E. S., Soares-Santos M., et al.,, MNRAS, 2018, vol. 474, p. 1361

Peterson J. R., Kahn S. M., et al.,, ApJ, 2003, vol. 590, p. 207

Peterson J. R., Paerels F. B. S., et al.,, A\&A, 2001, vol. 365, p. L104

Pierce C. M., Lotz J. M., et al., , MNRAS, 2010, vol. 408, p. 139

Rakos K. D., Schombert J. M., , ApJ, 1995, vol. 439, p. 47

Ruggiero R., Lima Neto G. B., , MNRAS, 2017, vol. 468, p. 4107

Rybicki G. B., Lightman A. P., Radiative Processes in Astrophysics, 1986, 400

Rykoff E. S., Rozo E., et al.,, ApJ, 2014, vol. 785, p. 104

Rykoff E. S., Rozo E., The DES Collaboration, ApJS, 2016, vol. 224, p. 1

Smith G. P., Treu T., et al., , ApJ, 2005, vol. 620, p. 78

Terlevich A. I., Kuntschner H., et al., , MNRAS, 1999, vol. 310, p. 445

The Dark Energy Survey Collaboration , vol. 9149, 2014, p. 91490V

The Dark Energy Survey Collaboration, ApJS, 2018, vol. 239, p. 18

Toomre A., . In Evolution of Galaxies and Stellar Populations , 1977, p. 401

Vijayaraghavan R., Ricker P. M., , MNRAS, 2013, vol. 435, p. 2713

Vikhlinin A., Burenin R. A., et al.,, ApJ, 2009, vol. 692, p. 1033

Vikhlinin A., Kravtsov A., et al., , ApJ, 2006, vol. 640, p. 691

Weston M. E., McIntosh D. H., et al., , MNRAS, 2017, vol. 464, p. 3882

White S. D. M., Frenk C. S., , ApJ, 1991, vol. 379, p. 52

White S. D. M., Rees M. J., , MNRAS, 1978, vol. 183, p. 341

Whitmore B. C., Gilmore D. M., Jones C., , ApJ, 1993, vol. 407, p. 489

Wright C. O., Brainerd T. G., , ApJ, 2000, vol. 534, p. 34

York D. G., SDSS Collaboration , AJ, 2000, vol. 120, p. 1579 
Apêndice 

Arquivo de texto Xpipe_Config.ini

[Paths]

outputPath: /data/des61.a/data/johnny/MaughanSample/

dataDownPath: /data/des61.a/data/johnny/chandraData/

[Files]

catInputFile: /data/des61.a/data/johnny/clusters/catalogs/maughan.fits

[Columns]

ID: Name

obsid: ObsID

ra: Xra

dec: Xdec

redshift: redshift

[Mode]

preProcess: False

imaging: True

preAnalysis: True

analysis: False

parallel: False

[PreProcess]

dowloadChandraObservation: True

reproData: True

flares: True

[Imaging]

fluxImage: True

blankField: True

pointSources: True

snapshot: True

energy: 0.7:2:1.5 \#\# energy_low:energy_high:energy_expmap

binSize: 4

[PreAnalysis]

maskPointSources: True

centerX: True

radialProfile: True

arf_rmf : True

[Analysis]

massX: True

csb: True 


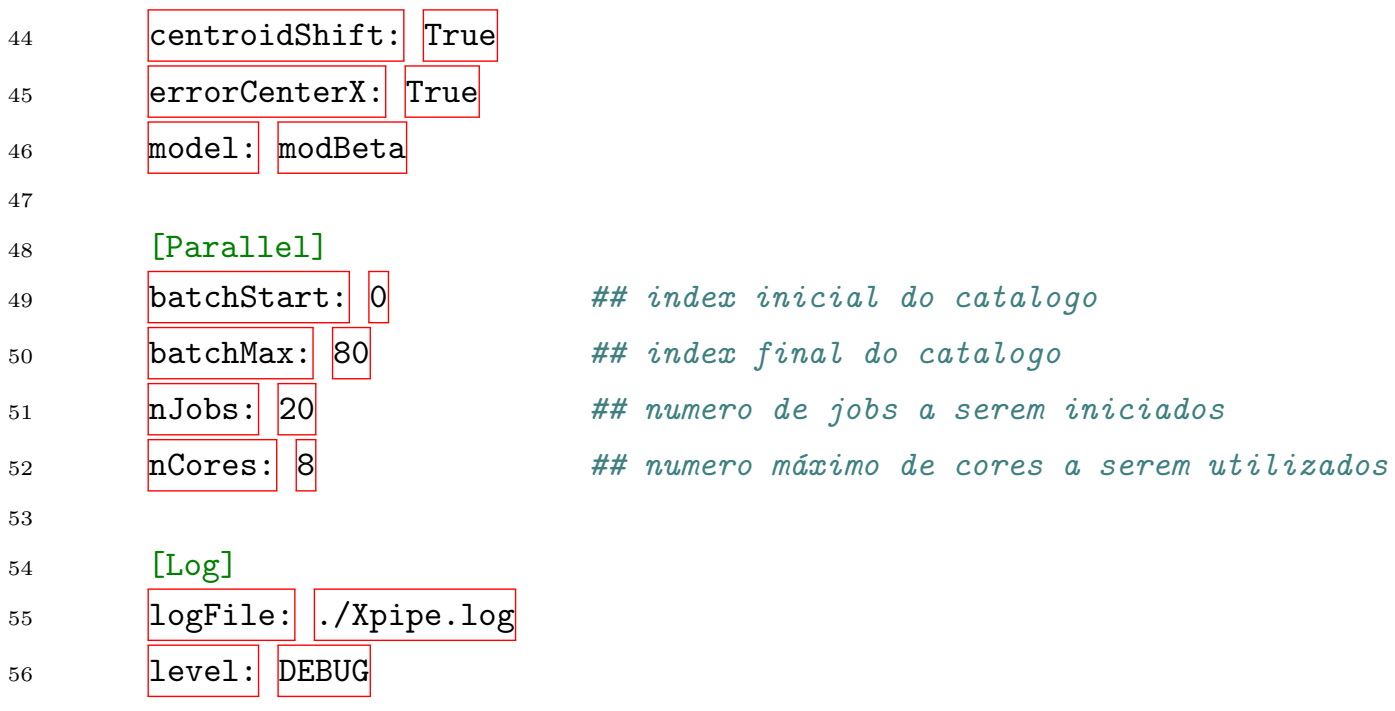

